UNIVERSIDAD POLITÉCNICA DE VALENCIA

Departamento de Matemática Aplicada

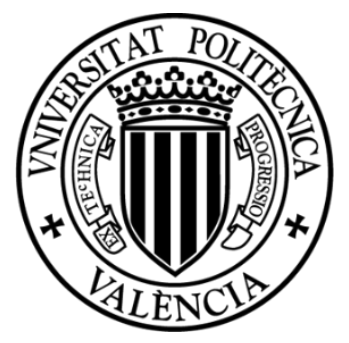

\title{
NUMERICAL METHODS FOR MULTIDISCIPLINARY FREE BOUNDARY PROBLEMS: NUMERICAL ANALYSIS AND COMPUTING
}

TESIS DOCTORAL

Presentada por:

Miguel Ángel Piqueras García

Dirigida por:

Dr. Lucas Jódar Sánchez

Dr. Rafael Company Rossi 



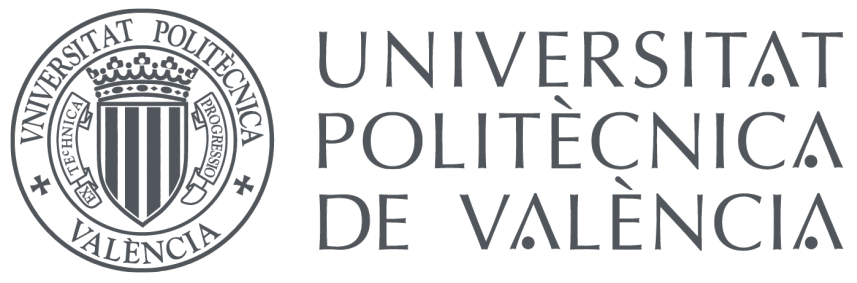

\section{NumericAl METHODS FOR MULTIDISCIPLINARY FREE BOUNDARY PROBLEMS: NUMERICAL ANALYSIS AND COMPUTING}

Tesis doctoral presentada por Miguel Ángel Piqueras García

Programa de Doctorado en Matemáticas

Departamento de Matemática Aplicada

Dirigida por Dr. Lucas A. Jódar Sánchez

y Dr. Rafael Company Rossi

El doctorando

El director

El director 

Numerical Methods for Multidisciplinary Free Boundary Problems: Numerical Analysis and Computing

Author: Miguel Ángel Piqueras García

Advisor: Lucas Jódar Sánchez

Advisor: Rafael Company Rossi

Text printed in Valencia

First edition, February 2018 

To my family 



\section{Resumen}

Multitud de problemas en ciencia e ingeniería se plantean como ecuaciones en derivadas parciales (EDPs). Si la frontera del recinto donde esas ecuaciones han de satisfacerse se desconoce a priori, se habla de "Problemas de frontera libre", propios de sistemas estacionarios no dependientes del tiempo, o bien de "Problemas de frontera móvil", asociados a problemas de evolución temporal, donde la frontera cambia con el tiempo. La solución a dichos problemas viene dada por la expresión de la(s) variable(s) dependiente(s) de la(s) EDP(s) junto con la función que determina la posición de la frontera. Dado que este tipo de problemas carece en la mayoría de los casos de solución analítica conocida, se hace preciso recurrir a métodos numéricos que permitan obtener una solución lo suficientemente aproximada a la exacta, y que además mantenga propiedades cualitativas de la solución del modelo continuo de $\operatorname{EDP}(\mathrm{s})$.

En este trabajo se ha abordado el estudio numérico de algunos problemas de frontera móvil provenientes de diversas disciplinas. La metodología aplicada consta de dos pasos sucesivos: en primer lugar, se aplica la llamada transformación de Landau o "Front-fixing transformation" al modelo en EDP(s) con el fin de mantener inmóvil la frontera del dominio; posteriormente, una vez planteado el nuevo problema en un recinto fijo, se procede a su discretización a través de un esquema en diferencias finitas. De ahí se obtienen esquemas numéricos que se implementan por medio de la herramienta informática MATLAB. Mediante un exhaustivo análisis numérico, se estudian propiedades del esquema y de la solución numérica (positividad, estabilidad, consistencia, monotonía, etc.).

En el primer capítulo de este trabajo se revisa el estado del arte del campo objeto de estudio, se justifica la necesidad de disponer de métodos numéricos adaptados 
a este tipo de problemas y se describe brevemente la metodología empleada en nuestro enfoque. Además, se exponen problemas particulares que se desarrollan en los capítulos siguientes.

El Capítulo 2 se dedica a un problema perteneciente a la Biología Matemática y que consiste en determinar la evolución en el tiempo de la distribución de la población de una especie invasora que se propaga en un hábitat y cuyo frente de propagación es desconocido. Este modelo consiste en una ecuación de difusiónreacción unida a una condición tipo Stefan, que relaciona las funciones solución y frontera móvil a determinar. Los resultados del análisis numérico confirman la existencia de una dicotomía propagación-extinción en la evolución a largo plazo de la densidad de población de la especie invasora, que depende de la relación entre los valores de los parámetros introducidos en el modelo. En particular, se ha podido precisar, por medio de experimentos numéricos, el valor del coeficiente de la condición de Stefan que separa el comportamiento de propagación del de extinción.

Los Capítulos 3 y 4 se centran en un problema de Química del Hormigón con interés en Ingeniería Civil: el proceso de carbonatación del hormigón, fenómeno evolutivo que lleva consigo la degradación progresiva de la estructura afectada y finalmente su ruina, si no se toman medidas preventivas. En el Capítulo 3 se considera un sistema de dos EDPs de tipo parabólico con dos incógnitas (concentraciones de dióxido de carbono disueltas en fases gaseosa y acuosa). Para su resolución, hay que considerar además, las condiciones iniciales, las de contorno y las de tipo Stefan (o de transmisión) en la frontera, que relacionan las concentraciones incógnitas y sus derivadas con la función frontera móvil o frente de avance de la zona carbonatada. Los resultados del análisis numérico se ajustan a los obtenidos en un estudio teórico previo. Se han llevado a cabo experimentos numéricos y se ha obtenido en términos cuantitativos la dinámica de las funciones solución y de la frontera, comprobando la tendencia a largo plazo de la ley de evolución de la frontera móvil hacia una función del tipo "raíz cuadrada del tiempo" por medio de un análisis de regresión. En el Capítulo 4 se considera un modelo más general y realista que el anterior, en el que intervienen seis especies químicas cuyas concentraciones hay que hallar, y que se encuentran tanto en la zona carbonatada como en la no carbonatada. 
En el Capítulo 5 se aborda un problema de transmisión de calor que aparece en diversos procesos industriales; en este caso, en el enfriamiento durante la colada de metal fundido, donde la fase sólida avanza y la líquida se va extinguiendo. La frontera móvil (frente de solidificación) separa ambas fases, siendo su posición en cada instante la variable a determinar, junto con las temperaturas en cada una de las dos fases. Después de la adecuada transformación y discretización, se implementa un esquema en diferencias finitas, subdividiendo el proceso en tres estadios temporales, a fin de tratar las singularidades asociadas a posiciones del frente móvil durante las etapas de inicialización y finalización. 



\section{Resum}

Multitud de problemes en ciència i enginyeria es plantegen com a equacions en derivades parcials (EDPs). Si la frontera del recinte on eixes equacions han de satisfer-se es desconeix a priori, es parla de "Problemas de frontera lliure", propis de sistemes estacionaris no dependents del temps, o bé de "Problemas de frontera mòbil", associats a problemes d'evolució temporal, on la frontera canvia amb el temps. La solució a dits problemes ve donada per l'expressió de la(les) variable(s) dependent(s) de la(les) EDP(s) junt amb la funció que determina la posició de la frontera. Atés que este tipus de problemes manca en la majoria dels casos de solució analítica coneguda, es fa precís recórrer a mètodes numèrics que permeten obtindre una solució prou aproximada a l'exacta, i que a més mantinga propietats qualitatives de la solució del model continu d'EDP(s).

En aquest treball s'ha abordat l'estudi numèric d'alguns problemes de frontera mòbil provinents de diverses disciplines. La metodologia aplicada consta de dos passos successius: en primer lloc, s'aplica l'anomenada transformació de Landau o "Front-fixing transformation" al model en EDP(s) a fi de mantindre immòbil la frontera del domini; posteriorment, una vegada plantejat el nou problema en un recinte fix, es procedix a la seva discretització a través d'un esquema en diferències finites. D'ací s'obtenen esquemes numèrics que s'implementen per mitjà de la ferramenta informàtica MATLAB. Per mitjà d'una exhaustiva anàlisi numèrica, s'estudien propietats de l'esquema i de la solució numèrica (positivitat, estabilitat, consistència, monotonia, etc.).

En el primer capítol d'aquest treball es revisa l'estat de l'art del camp objecte d'estudi, es justifica la necessitat de disposar de mètodes numèrics adaptats a aquest tipus de problemes i es descriu breument la metodologia emprada en el nostre 
enfocament. A més, s'exposen problemes particulars que es desenrotllen en els capítols següents.

El Capítol 2 es dedica a un problema pertanyent a la Biologia Matemàtica i que consistix a determinar l'evolució en el temps de la distribució de la població d'una espècie invasora que es propaga en un hàbitat i el front de propagació el qual és desconegut. Este model consistix en una equació de difusió-reacció unida a una condició tipus Stefan, que relaciona les funcions solució i frontera mòbil a determinar. Els resultats de l'anàlisi numèrica confirmen l'existència d'una dicotomia propagació-extinció en l'evolució a llarg termini de la densitat de població de l'espècie invasora, que depén de la relació entre els valors dels paràmetres introduïts en el model. En particular, s'ha pogut precisar, per mitjà d'experiments numèrics, el valor del coeficient de la condició de Stefan que separa el comportament de propagació del d'extinció.

Els Capítols 3 i 4 se centren en un problema de Química del Formigó amb interés en Enginyeria Civil: el procés de carbonatació del formigó, fenomen evolutiu que comporta la degradació progressiva de l'estructura afectada i finalment la seua rü̈na, si no es prenen mesures preventives. En el Capítol 3 es considera un sistema de dos EDPs de tipus parabòlic amb dos incògnites (concentracions de diòxid de carboni dissoltes en fases gasosa i aquosa). Per a la seua resolució, cal considerar a més, les condicions inicials, les de contorn i les de tipus Stefan (o de transmissió) en la frontera, que relacionen les concentracions incògnites i les seues derivades amb la funció frontera mòbil o front d'avanç de la zona carbonatada. Els resultats de l'anàlisi numèrica s'ajusten als obtinguts en un estudi teòric previ. S'han dut a terme experiments numèrics i s'ha obtingut en termes quantitatius la dinàmica de les funcions solució i de la frontera, comprovant la tendència a llarg termini de la llei d'evolució de la frontera mòbil cap a una funció del tipus "arrel quadrada del temps" per mitjà d'una anàlisi de regressió. En el Capítol 4 es considera un model més general i realista que l'anterior, en el que intervenen sis espècies químiques les concentracions de les quals cal trobar, i que es troben tant en la zona carbonatada com en la no carbonatada.

En el Capítol 5 s'aborda un problema de transmissió de calor que apareix en diversos processos industrials; en aquest cas, en el refredament durant la bugada de metall fos, on la fase sòlida avança i la líquida es va extingint. La frontera mòbil 
(front de solidificació) separa ambdues fases, sent la seua posició en cada instant la variable a determinar, junt amb les temperatures en cada una de les dos fases. Després de l'adequada transformació i discretització, s'implementa un esquema en diferències finites, subdividint el procés en tres estadis temporals, per tal de tractar les singularitats associades a posicions del front mòbil durant les etapes d'inicialització i finalització. 



\section{Abstract}

Many problems in science and engineering are formulated as partial differential equations (PDEs). If the boundary of the domain where these equations are to be solved is not known a priori, we face "Free-boundary problems", which are characteristic of non-time dependent stationary systems; besides, we have "Movingboundary problems" in temporal evolution processes, where the border changes over time. The solution to these problems is given by the expression of the dependent variable(s) of $\operatorname{PDE}(\mathrm{s})$, together with the function that determines the position of the boundary. Since the analytical solution of this type of problems is lacked in most cases, it is necessary to resort to numerical methods that allow an accurate enough solution to be obtained, and which also maintain the qualitative properties of the solution(s) of the continuous model.

This work approaches the numerical study of some moving-boundary problems that arise in different disciplines. The applied methodology consists of two successive steps: firstly, the so-called Landau transformation, or "Front-fixing transformation", which is used in the PDE(s) model to maintain the boundary of the domain immobile; later, once the new problem is presented in a fixed region, we proceed to its discretization with a finite difference scheme. Different numerical schemes are obtained and implemented through the MATLAB computational tool. Properties of the scheme and the numerical solution (positivity, stability, consistency, monotonicity, etc.) are studied by an exhaustive numerical analysis.

The first chapter of this work reports the state of the art of the field under study, justifies the need to adapt numerical methods to this type of problem, and briefly describes the methodology used in our approach. Particular problems are presented and dealt with in the following chapters. 
Chapter 2 presents a problem in Mathematical Biology that consists in determining over time the evolution of the distribution of an invasive species population that spreads in a habitat, whose propagation front is unknown. This problem is modelled by a diffusion-reaction equation linked to a Stefan-type condition, which relates the solution and boundary functions to be determined. The results of the numerical analysis confirm the existence of a spreading-vanishing dichotomy in the long-term evolution of the population density of the invasive species, which depends on the relation between the values of the parameters introduced into the model. In particular, it is possible to determine by means of numerical experiments the value of the coefficient of the Stefan condition that separates the propagation behaviour from extinction.

Chapters 3 and 4 focus on a problem of Concrete Chemistry with an interest in Civil Engineering: the carbonation of concrete, an evolutionary phenomenon that leads to the progressive degradation of the affected structure and its eventual ruin if preventive measures are not taken. Chapter 3 considers a system of two parabolic type PDEs with two unknowns (concentrations of dissolved carbon dioxide in gaseous and aqueous phases). For its resolution, the initial and boundary conditions have to be considered together with the Stefan (or transmission) conditions on the carbonation front, which relate the unknown concentrations and their derivatives with the moving boundary or advancing front of the carbonated zone. The numerical analysis results agree with those obtained in a previous theoretical study. Numerical experiments are run and the dynamics of the concentrations solution and the moving boundary are qualitatively by performing a regression analysis to verify the long-term behaviour of the law of evolution of the moving boundary as a "square root of time". Chapter 4 considers a more general and realistic model than the previous one, which includes six chemical species, defined in both the carbonated and non-carbonated zones, whose concentrations have to be found.

Chapter 5 addresses a heat transfer problem that appears in various industrial processes; in this case, the solidification of metals in casting processes, where the solid phase advances and liquid reduces until it is depleted. The moving boundary (the solidification front) separates both phases. Its position in each instant is the variable to be determined together with the temperature profiles in both phases. 
After suitable transformation, discretization is carried out to obtain a finite difference scheme to be implemented. The process was subdivided into three temporal stages to deal with the singularities associated with the moving boundary position in the initialisation and depletion stages. 



\section{Acknowledgements}

I would like to use this opportunity to express my gratitude to everyone who supported me throughout the elaboration of this thesis. In particular, I am indebted to my supervisors, Professor Dr. Lucas A. Jódar Sánchez and Professor Dr. Rafael Company Rossi from the Institute of Multidisciplinary Mathematics at the Polytechnic University of Valencia. I am very thankful for their inspiring guidance, constructive objections and wise advice during these years. I am sincerely grateful to them for sharing their knowledge and expertise, particularly in numerical analisys and, generally, in applied mathematics.

I would also like to thank all my colleagues at the Institute of Multidisciplinary Mathematics for their exceptional mixture of friendship, helpfulness and team spirit.

Last but not the least, I am so grateful to family, to whom I dedicate all the efforts made to write this thesis, from the beginning to the end.

Miguel Ángel

February 2018 


\section{List of Publications}

\section{Published Papers}

M.-A. Piqueras, R. Company, L. Jódar, Numerical analysis and computing of free boundary problems for concrete carbonation chemical corrosion, Journal of Computational and Applied Mathematics, 336:297-316, 2018.

DOI:10.1016/j.cam.2017.12.036

M.-A. Piqueras, R. Company, L. Jódar, Computing positive stable numerical solutions of moving boundary problems for concrete carbonation, Journal of Computational and Applied Mathematics, 330:794-805, 2018.

DOI:10.1016/j.cam.2017.03.007

M.-A. Piqueras, R. Company, L. Jódar, A front-fixing numerical method for a free boundary nonlinear diffusion logistic population model, Journal of Computational and Applied Mathematics, 309:473-481, 2017.

DOI:10.1016/j.cam.2016.02.029

\section{Submitted Papers}

M.-A. Piqueras, R. Company, L. Jódar, Stable and monotone numerical solution of two-phase freezing Stefan problems, submitted to Journal of Computational and Applied Mathematics, 2017. 


\section{Presentations in Conferences}

M.-A. Piqueras, R. Company, L. Jódar, ”Numerical solution of nonlinear moving boundary problems for carbonation in reinforced concrete structures", Mathematical Modelling in Engineering Human Behaviour 2017 Conference, July 17-19, 2017, Instituto de Matemática Multidisciplinar UPV, Valencia, Spain. Proceedings of the Conference, ISBN 978-84-697-8505-8, pp. 250-255.

M.-A. Piqueras, R. Company, L. Jódar, "Solving moving boundary concrete carbonation models", 2nd International Conference on Computer, Network Security and Communication Engineering (CNSCE 2017), March 26-27, 2017, Bangkok, Thailand. ISBN 978-1-60595-439-4, pp. 21-27.

M.-A. Piqueras, R. Company, L. Jódar, "Positive stable numerical solutions of moving boundary problems for concrete carbonation", Mathematical Modelling in Engineering Human Behaviour 2016 Conference, July 18-20, 2016, Instituto de Matemática Multidisciplinar UPV, Valencia, Spain. Proceedings of the Conference, ISBN 978-84-617-7205-6, pp. 206-212.

M.-A. Piqueras, R. Company, L. Jódar, ”A front-fixing numerical method for a free boundary nonlinear diffusion logistic population model", Mathematical Modelling in Engineering Human Behaviour 2015 Conference, September 9-11, 2015, Instituto de Matemática Multidisciplinar UPV, Valencia, Spain. Proceedings of the Conference, ISBN 978-84-608-5355-8, pp. 255-260.

\section{Technical Reports}

M.-A. Piqueras, "Problemas de frontera libre y móvil: algunas aplicaciones recientes en modelización matemática", June 2016. 



\section{Contents}

List of Figures

Xxi

List of Tables

xxiii

1 Introduction 1

1.1 Background, motivation and methodology . . . . . . . . . 11

1.2 Three real cases of interest . . . . . . . . . . 5

1.2.1 Population dynamics of an invasive species . . . . . . 6

1.2.2 Damage by carbonation of reinforced concrete structures . [7]

1.2.2.1 Two-species carbonation model . . . . . . . 8

1.2.2.2 Six-species carbonation model ....... 9

1.2.3 Phase change processes . . . . . . . . . . . 10

1.3 Numerical treatment of moving boundary problems . . . . . . 12

1.3.1 Finite difference method . . . . . . . . . . . . 12

1.3.2 Preliminary concepts of numerical analysis . . . . . 16

2 Population dynamics of an invasive species 19

2.1 Transformation and discretization of the continuous problem ... 21]

2.2 Consistency . . . . . . . . . . . . . . . . . . . . . . 24

2.3 Positivity and Stability . . . . . . . . . . . . 26

2.3 .1 Positivity ..................... 26

2.3.2 Stability . . . . . . . . . . . . . 28 28

2.4 Numerical dichotomy: spreading versus vanishing . . . . . . . . 31

2.5 Conclusions . . . . . . . . . . . . . . . . . . 34 
32 species model for damage by carbonation of reinforced concrete structures 35

3.1 Front-fixing transformation and discretization . . . . . . . 38

3.2 Positivity, stability and monotonicity of the numerical solution . . 41]

3.3 Numerical evidence of the $\sqrt{t}$-law of propagation . . . . . . . 50

3.4 Consistency ................... 51

46 species model for damage by carbonation of reinforced concrete structures

4.1 Front-fixing transformation . . . . . . . . . . . . 62

4.2 Discretization and numerical scheme construction . . . . . . . 64

4.3 Numerical analysis: stability and positivity . . . . . . . 72

4.4 Monotonicity of the numerical solution . . . . . . . . 82

4.5 Conclusions ..................... 87

5 Phase change processes 91

5.1 Front-fixing transformation and numerical modelling . . . . . 95

5.1.1 First stage numerical modelling . . . . . . . . . . . 97

5.1.2 Second stage numerical modelling . . . . . . . . . 102

5.1.3 Third stage numerical modelling . . . . . . . . . 106

6 Conclusions

Bibliography 


\section{List of Figures}

2.1 Numerical solution of Example 1 for $z=0.5$ under stability condition 30

2.2 Numerical solution of Example 2 for $z=0.5$ when the stability condition is broken. . . . . . . . . . . . . . . 30

2.3 Numerical solution of Example 3 for several values of time in a spreading case. . . . . . . . . . . . . . . 31

2.4 Numerical solution of Example 4 for several values of time in a vanishing case. . . . . . . . . . . . . . 32

2.5 Expanding front $H(t)$ of Example 5 for several values of $\mu$. . . 33

2.6 Expanding front speed $d H / d t$ of Example 6 for several values of $\mu$. 34

3.1 Numerical solution of Example 1 for $t=0.375$ years, when positivity condition is broken. . . . . . . . . . 45

3.2 Numerical solution of $U(t, x)$ and $V(t, x)$ in Example 2 for $t=13$ years, under stability condition. . . . . . . . . . . . 48

3.3 Numerical solution $U(t, x)$ of Example 3 for several equidistant times $t_{i} \ldots \ldots \ldots \ldots \ldots \ldots$. . . . . . . . . . . . . .

3.4 Numerical solution $V(t, x)$ of Example 3 for several equidistant times $t_{i} \ldots \ldots \ldots \ldots \ldots \ldots$. . . . . . . . . . . . . .

4.1 Numerical solution $\bar{U}_{1}(x, t)$ of Example 1 for $t=0.95$ years, when positivity condition is broken. . . . . . . . . . . 81

4.2 Numerical concentrations $\bar{U}_{i}(x, t), i \in\{1,2,5\}$, and $\bar{U}_{i}(x, t), i \in$ $\{3,6\}$, in Example 2 for $t=9$ years, under stability condition. . . 
4.3 Position of the numerical carbonation front $\sqrt{r^{n}}$, and values of $\rho_{2, n}$, in Example 2, as a function of time. . . . . . . . . 82

4.4 Numerical solution $\bar{U}_{1}(x, t)$ of Example 3, for several equidistant times. . . . . . . . . . . . . . 86

4.5 Numerical solution $\bar{U}_{3}(x, t)$ of Example 3, for several equidistant times. ................... 87

5.1 Numerical solutions $\hat{U}_{s}(y, \tau), \hat{U}_{l}(y, \tau)$ at the end of the first stage. 102

5.2 Numerical solutions $U_{s}(z, \tau), U_{l}(z, \tau)$ at the end of the second stage.106

5.3 Numerical solutions $\hat{U}_{s}(y, \tau), \hat{U}_{l}(y, \tau)$ for different fractions of the extinction time $t_{e}$. Dash-dot lines represent the solid phase and continuous lines the liquid phase. . . . . . . . . . . . . 107

5.4 Numerical solidification front $\tilde{S}(\tilde{t})$ as a function of time. . . . . . 108

5.5 Above: Spatial stages distribution. Below: Time stages distribution. 109 


\section{List of Tables}

2.1 Associated CPU time for several values of $T$ (Example 4). . . . 32

3.1 Data for numerical examples. . . . . . . . . . . . . . 46

3.2 Carbonation depth for several times. . . . . . . . . 51

4.2 Dependence of process time on the temporal step size. . . . . 8 82

4.1 Data for numerical examples. . . . . . . . . . . . . 89

5.1 Dependence of $C$ on the step sizes when the parameters of the model are $\kappa=K=\beta=1 \ldots \ldots \ldots$. . . . . . . . . . . . . . .

5.2 Dependence of $t_{0}, t_{1}, t_{e}$ and RMSE of temperatures on the fraction $R_{0}$, when the parameters of the model are $\kappa=K=\beta=1$. . . 109 



\section{Introduction}

\subsection{Background, motivation and methodology}

Many phenomena and processes in science, engineering and industry are governed by partial differential equations (PDEs) that are posed on a domain whose boundary is not known a priori and has to be found as part of the solution. According to [26], such problems can be split into two groups: free- and moving-boundary problems. The first group arises in stationary or steady-state problems, where variables remain unchanged with time and the boundary is immobile. In contrast, movingboundary problems are associated with evolution problems that model a system which changes with time, whose unknown boundaries depend on both space and time.

Frequently, problems in the second group are referred to as Stefan problems given the Stefan condition that links the boundary with the unknown solution of the PDE or system of PDEs. However, using the term free-boundary has become commonplace in the scientific community regardless of the problem's time dependency. Throughout this work the expression free-boundary may apply to both categories of problems.

In recent years, many phenomena of practical interest from different disciplines have been modelled as free-boundary problems. In these problems, the free 
boundary describes different quantities, such as the spreading front over which the population of a certain invasive biological species advances through a habitat, the front line of human migrations for social, economical, political or environmental reasons, the border of the geographical area where a technological innovation has been disseminated, the limit, measured in "cyber-distances" into which a relevant news in social networks extends, the advance of healing in corneal epithelial tissue after a wound occurs, the propagation front of natural phenomena, such as tsunamis, forest fires and avalanches of snow, the extent of a polluted atmospheric region or a contaminated hydrological system, the optimal exercise boundary of American option prices, the speculative part in price rises in financial bubbles, the propagation of braking on road stretches with congested round traffic, depth from the outer face to that which penetrates the chemical phenomena of carbonation in concrete infrastructures and buildings, the separating surface between plasma and vacuum zones inside some types of experimental magnetic confinement fusion reactors and the interface between two fluids in a porous medium. In addition, optimal stopping problems that arise in the decision theory are closely connected to free-boundary problems.

In such problems, the location of the moving boundary must be determined together with the solution of the PDE or system of PDEs. The analytical solution of such problems is seldom available. As a paradigmatic case of a Stefan problem with a known exact solution, one noteworthy case is modelling the phase change that occurs during a substance freezing process which extends over a onedimensional semi-infinite region, whose initial constant temperature is above the melting point (initial condition), on the edge of which the freezing temperature is imposed (boundary condition). This problem was originally formulated in 1831 by G. Lamé and E. Clapeyron, see [48] and [26], Chapter 3. During a series of lectures in the early 1860s at Königsberg, C.G. Neumann found and presented the exact solution in terms of error and complementary error functions ([66]). However, it was not until 1889 when it was presented by J. Stefan in a study on polar ice thickness, [74]. Some other one-dimensional problems, for which the solution in a closed form can be found, have unbounded domains, quite simple initial and boundary conditions, and the parameters of the mathematical model, such as 


\subsection{Background, motivation and methodology}

thermal conductivity, must be accepted as constants. Ordinarily, these solutions are functions of the variable $x / \sqrt{t}$, the so-called similarity solutions (see [12]).

This work is about the construction, analysis and computing of numerical methods to solve reaction-diffusion moving-boundary problems. The solution technique involves two main sequential steps:

1. The well-known Landau transformation (see [26, 49]) is applied to the original problem. By immobilizing the moving boundary, this transform allows a new equivalent problem to be obtained in which the resulting PDE or PDE system is generally less simple than it is in the original formulation. Notwithstanding, the transformed problem is tractable because it is posed in a fixed domain.

2. The resulting PDE problem is treated numerically by the finite difference method. Thus an explicit or implicit numerical scheme is obtained as a basis for computations, which are made by implementing Matlab codes. The importance attached to the reliability of these schemes must be emphasized as properties such as stability and consistency which are guaranteed, in all cases, by an exhaustive numerical analysis.

At present, finite differences, finite elements and boundary elements are the principal methods used to numerically solve both steady-state and transient freeor moving-boundary PDE problems. This thesis focuses on the finite difference technique. This approach can be implemented in two main ways: using the fixed domain method or the variable domain method. The latter can be divided into two subgroups: the front-tracking and the front-fixing method. The fixed domain method considers of the entire domain, which includes all the regions together. The problem is reformulated in such a way that the Stefan condition implicitly applies to the whole fixed domain. The position of the moving boundary appears a posteriori as one feature of the solution. This method is easy to implement because tracking the movement of the moving boundary is not necessary (see [39]).

In the second group, the front-tracking method consists of evaluating the position of the moving boundary in each time step. The boundary generally lies between two grid points at any given time. The grid itself has to be deformed 
so that the moving boundary is always on a grid line. In the front-fixing approach, the moving boundary is immobilized by using a transformation in the spatial variable; for instance, the so-called Landau transformation. The price paid to use this technique is that the new equations usually present more terms than the original ones being transformed. 


\subsection{Three real cases of interest}

Moving-boundary problems play a crucial role in modelling many natural phenomena in science and a wide variety of processes involved in engineering and industry. Furthermore, many other current problems that have been studied with no adequate treatment are expected to be modelled in the future as free- or movingboundary problems. As we stated before, this approach allows the exact solution to be obtained in only a few particular simple cases. Hence it is necessary to resort to numerical and computational methods to obtain a sufficiently approximate solution.

In this section, we present three particular problems from the fields of ecology, construction industry (civil engineering and building construction) and metallurgy, and each field is dealt with in a separate chapter in this work. By applying our approach to perform their numerical analysis, we can obtain accurate enough numerical solutions and their qualitative properties, which are compared with the theoretical results provided by the authors who have worked with continuous models.

What these cases share, besides the fact that they are modelled by movingboundary problems, is that they are evolution problems in which the governing PDEs are of reaction-diffusion type, in which the initial and boundary conditions are prescribed together with some Stefan-like constraints. In all cases, the moving boundary represents some physical quantity of practical interest. Following our methodology, in the first step we use the well-known Landau transformation (see [26, 49]) to convert the continuous PDE problem into a fixed spatial domain one, in which the position of the moving boundary is included as an additional unknown variable. Subsequently, the discretization of the transformed problem is implemented and a finite difference scheme is obtained. Apart from the computation of the solution and the position of the free boundary, a numerical analysis is run to study the properties of the finite difference scheme and the approximate solution, such as stability and consistency. The positivity of the solution is also studied in all cases, because they represent quantities, such as population densities, mass concentrations and temperatures, which need to be positive. 


\subsubsection{Population dynamics of an invasive species}

The first example of implementing our approach consists of a moving-boundary problem that arises in those problems that model biological invasions. The onedimensional spatial-temporal spreading of an invasive species in a habitat is modelled by a moving-boundary diffusion logistic partial differential problem, in which the moving-boundary represents the a priori unknown expanding front of the species.

The pioneering work in this field was initiated in 1937 independently by Fisher (see [34]) and Kolmogorov-Petrovsky-Piskunov (KPP) (see [43]) in which the PDE problem had to be solved over an infinite domain with no boundary constraints. Recently, Du and Lin [30] approached the spreading population problem by considering it a free-boundary problem under a Stefan condition, which linked the population density gradient at the spreading front and the speed of the moving boundary. The diffusive PDE problem for the population density of the invasive species $U(t, x)$ depends on time $t$ and the spatial variable $x$ and is written as follows:

$$
\frac{\partial U}{\partial t}-D \frac{\partial^{2} U}{\partial x^{2}}=U(a-b U), t>0,0<x<H(t),
$$

satisfying the Stefan condition

$$
H^{\prime}(t)=-\mu \frac{\partial U}{\partial x}(t, H(t)), \quad t>0
$$

with the corresponding initial and boundary conditions. The latter, formulated as a homogeneous Neumann boundary condition at $x=0$, indicates that the left boundary is fixed given the presence, for instance, of a natural obstacle to spreading, with the population confined to its right side.

Here $H(t)$ is the unknown moving boundary, where the population is distributed within the interval $[0, H(t)], D$ is the dispersal rate and parameters $a$ and $b$ respectively refer to the intrinsic growth rate and the competition between individuals of the invasive species. Unlike previous models, in which only spreading behaviour is permissible, the authors of [30] showed that dichotomic behaviour exists for long times: the population vanishes or spreads to the entire infinite habitat with a density value that approaches the habitat carrying capacity $a / b$. Behaviour depends on the 
initial front position and population density, and also on the parameter $\mu$ value that appears in the Stefan condition. There is a threshold parameter $\mu^{*}$, whose value is unknown in advance, which determines the vanishing or spreading alternative.

\subsubsection{Damage by carbonation of reinforced concrete structures}

Concrete carbonation problems arise in the civil engineering and building construction fields as this chemical process involves a major cause of damage of reinforced concrete structures. This is a gradual phenomenon, according to which the $p H$ level, initially high with a value around 13-14, progressively lowers. This drop in $p H$ leads to the corrosion of the embedded steel reinforcement. With time, spalling and cracks appear in the concrete structure since the products of corrosion occupy a bigger volume than the original steel reinforcement, and the internal pressure increases. As a result, the structure's functionality and durability are seriously affected. This process is enhanced in urban and industrial areas, or generally in structures exposed to environments with high carbon dioxide concentrations. A good understanding of the carbonation process evolution is crucial to predict the life service of concrete structures and to save large amounts of money and energy.

Carbon dioxide in the gaseous phase is transported through unsaturated concrete pores by diffusion and dissolves (according to Henry's law) in the aqueous phase, where it is further transported towards the place where the carbonation reaction takes place. The other reactant, calcium hydroxide, is initially in the solid matrix of cement. By means of a dissolution process, it arrives at the aqueous phase of pores and reacts with the carbon dioxide present in the aqueous phase. Reaction products are water together with calcium carbonate. Gradually the process penetrates more deeply into concrete, and a carbonation front appears which separates the carbonated zone from the uncarbonated one.

Modelling the concrete carbonation process can be simplified to a single reaction of atmospheric carbon dioxide with the calcium hydroxide found in the pore solution to form water and calcium carbonate. We also assume that the values of both the material (composition of cement and concrete, porosity and pore sizes) and environmental conditions (carbon dioxide concentration, relative humidity, temperature) remain unchanged in all the process stages. 
The reaction mechanism is as follows: atmospheric carbon dioxide diffuses through the unsaturated concrete matrix and dissolves into the pore water, while calcium hydroxide is available in the pore solution. Calcium carbonate precipitates to the solid cement matrix. To model these processes, in this thesis we consider two different models that describe the involved chemistry and transport. In both models, a set of nonlinear parabolic reaction-diffusion equations describe the chemical reactions and transport phenomena which come into play. Taking into account the complexity of the real phenomenon and the presence of a large number of parameters, with these simplified models we can capture the basics of the carbonation mechanism and accurately predict the carbon dioxide penetration depth.

\subsubsection{Two-species carbonation model}

The first of the models we dealt with has been presented in [2, 3], where the authors study a one-dimensional moving-boundary problem by modelling the carbonation process. The unknowns are carbon dioxide mass concentrations in the air and water phases of pores, respectively denoted by $U(t, x)$ and $V(t, x)$, depending on variables time $t$ and space $x$. As usual, the position of the carbonation front $S(t)$ also has to be determined. The temporal variable ranges from $t=0$ to the time horizon $T>0$, while the space variable is measured from the exposed boundary $x=0$ to the unknown carbonation front $x=S(t)$.

The model is described by the coupled system of two parabolic reaction-diffusion PDEs:

$$
\begin{gathered}
\frac{\partial U}{\partial t}-\frac{\partial}{\partial x}\left(\kappa_{1} \frac{\partial U}{\partial x}\right)=f(U, V), \quad 0<t<T, \quad 0<x<S(t), \\
\frac{\partial V}{\partial t}-\frac{\partial}{\partial x}\left(\kappa_{2} \frac{\partial V}{\partial x}\right)=-f(U, V), \quad 0<t<T, \quad 0<x<S(t) .
\end{gathered}
$$

Here $\kappa_{1}$ and $\kappa_{2}$ are diffusion constants and reaction terms $\pm f(U, V)$ involve Henry's laws. The left boundary conditions are prescribed for both concentrations at the exposed boundary, i.e. at $x=0$. The initial conditions are given by concentrations and position of the carbonation front for $t=0$. The propagation front comes from the Stefan-like conditions, which establish the dependence among the chemical species' concentrations, their gradients and the speed at which the carbonated region moves through the concrete element. 
The cited authors rigorously state the asymptotic behaviour, as previously evidenced empirically, of the carbonation front: for long times, a $\sqrt{t}$-law exists for the penetration depth, that is $S(t) \approx C \sqrt{t}$, for some constant $C>0$.

\subsubsection{Six-species carbonation model}

Another recent different and more sophisticated model for concrete carbonation is exposed in [60], in which not only the carbonated zone $\Omega_{1}(t)$ is considered, but also the uncarbonated zone $\Omega_{2}(t)$ beyond the carbonation front and more chemical species involved in the chemical process are also taken into account. The concrete element is assumed to be a sample with a thickness that equals $L$. Space variable $x$ is measured from exposed boundary $x=0$ to sealed boundary $x=L$, and the unknown carbonation front or moving boundary is denoted by $x=S(t)$, which satisfies $0<S(t)<L$. The chemical species present in $\Omega_{1}(t)$ are carbon dioxide in both gaseous and in aqueous, calcium carbonate and water content, while the substances considered in $\Omega_{2}(t)$ are carbon dioxide and water.

The continuous model applied in [59, 60] is described by a system of five PDEs, along with one ordinary differential equation, and involves the unknown mass concentrations as reaction-diffusion equations. The reaction or production terms for each equation correspond to Henry's law for carbon dioxide in the gaseous and liquid phases, the dissolution rates of calcium hydroxide and the production rates of calcium carbonate and water at the carbonation front, and are zero for the equations about water content.

The initial location of the front is $S(0)=S_{0}>0$ and the initial concentrations of the different chemical species in their respective domains constitute the initial conditions. The boundary conditions at exposed boundary $x=0$ and sealed boundary $x=L$ are respectively determined by the mass concentrations in the exterior environment and on the surface of the concrete element, which is protected against the effect of carbon dioxide. At free boundary $x=S(t), 0<t<T$ is a set of Rankine-Hugoniot transmission conditions that are imposed, and another equation about the dynamics of the carbonation front is regarded. 


\subsubsection{Phase change processes}

Solid-liquid phase changes appear frequently in nature. Knowledge on them has become crucial in both pure sciences such as astrophysics, geophysics and meteorology, and different engineering and technology branches; e.g., chemical engineering (latent heat storage systems, sea water desalination), civil engineering (ground freezing for construction, concrete hardening, permafrost), electrical engineering (melting of safety fuses), aeronautical and aerospace engineering (freezing of fuel in air and spacecraft or ablation of bodies in atmospheric re-entry), metallurgical engineering (solidification of metals during continuous casting processes), nuclear engineering (in both fission and experimental fusion reactors), etc. In industry, many products and materials (metals, ceramics, polymers, electronic components, processed foods) undergo a solid-liquid phase change process some time in their lifetime, hence the importance of controlling these phenomena.

The phase change process is modelled as a particular case of the heat conduction problem, known in the literature as the Stefan problem, which involves melting (or solidification) of a material. In a one-dimensional two-phase Stefan problem, at time $t=0$ the solid (liquid) occupies finite $0 \leq x \leq a$ or semi-infinite region $x \geq 0$. Since the mathematical structure of the melting and solidification problem is analogous, and the treatment of both problems is the same, we henceforth will focus on the latter. At the initial time, the temperature of the material is uniform, $T_{h}$, which is higher than the phase-change temperature, and is written as $T_{f}$. The material is cooled at point $x=0$ by imposing constant temperature $T_{c}$, which is lower than the phase-change temperature $T_{f}$. With time, the phase interface advances and the domain consists of a solid and a liquid region separated by a sharp moving interface $S(t)$, which coincides with the phase change temperature isotherm.

In the finite domain case, the solidification of an initially liquid substance is governed by two equations, denoted by subscript $s$ for the solid phase and $l$ for the liquid phase, both of the type ([57]):

$$
\rho c_{i} \frac{\partial T_{i}}{\partial t}=\kappa_{i} \frac{\partial^{2} T_{i}}{\partial x^{2}}, \quad 0<x<S(t), \quad t>0, \quad i \in\{s, l\} .
$$

For the solid phase, $T_{s}=T_{s}(x, t)$ indicates temperature, $\kappa_{s}$ denotes thermal conductivity and $c_{s}$ refers to the specific heat capacity. The same notation is used 
for the liquid phase by exchanging subscript $s$ for $l$. The material's mass density is assumed to have the same value $\rho$ in both the solid and liquid states.

Boundary, initial and Stefan conditions are added to the above equations: boundary conditions, which are obtained from imposing a value for the cooling temperature at one of the edges of the element (Dirichlet condition) and a perfect thermal insulation in the other boundary (Neumann condition), together with the isothermal relations at the moving boundary. The initial conditions are given by the initial temperatures profile of the element. Finally, the Stefan condition expresses the heat balance at the interface between the solid and liquid phases, and exhibits the solidification front dynamics. It takes this form:

$$
\rho C_{m} \frac{d S}{d t}=\kappa_{s} \frac{\partial T_{s}}{\partial x}-\kappa_{l} \frac{\partial T_{l}}{\partial x}, \quad x=S(t), \quad t>0,
$$

where value $C_{m}$ represents the latent heat of fusion.

In this model, we consider that physical parameters, i.e. density, specific heat capacity, thermal conductivity and latent heat are constant throughout the process and their values remaining unaltered in each phase. As mentioned in reference [26], the problems problems in which a difference in density is assumed between both phases incorporate an additional advection transport term in the heat transfer equations. 


\subsection{Numerical treatment of moving boundary prob-} lems

Finite difference is one of the oldest and most straightforward methods to solve differential equations. With the appearance of computers and the software implementation of finite difference algorithms in the 1950s, the method received a decisive boost, and it was possible to address and numerically solve a wide variety of science and engineering problems in a relatively short calculation time. In the last few decades, interest of the finite difference method has increased, together with other alternative numerical techniques (finite element, finite volume, etc.). However, the finite difference approach has the advantage of relative simplicity, ease of implementing and economy of resources from a computational point of view (especially when working with explicit schemes), apart from the major theoretical advances that this branch of numerical analysis has made in recent years.

This section reports the basics of the finite difference method which, in conjunction with front-fixing transformation, is resorted to in this work. It also presents general concepts like truncation error, consistency and stability. As pointed out above, our approach to treat moving-boundary problems comprises two successive steps. Firstly, the front-fixing Landau transform is applied to the original problem, which leads to a new problem posed in a spatial fixed domain. Secondly, this problem is discretized by replacing the continuous domain with the independent variables of PDE(s) by a set of points in a grid in which the dependent variables are approximated.

\subsubsection{Finite difference method}

As previously mentioned, most PDEs problems have no analytical solution; i.e. a function that solves the equation or the system of equations, plus a general set of initial and/or boundary conditions. In this work, we focus on a class of such problems; evolution problems that are formulated as second-order PDEs of parabolic type with moving boundaries. Hence the solution to the problem must fulfil both the initial and boundary conditions, as well as the constraint imposed at the moving boundary, which usually comes in the form of one Stefan-like condition or 


\subsection{Numerical treatment of moving boundary problems}

more. Thus for practical purposes, finding an approximate solution by numerical procedures is mandatory.

The finite difference method consists in estimating the solution of an ordinary or partial differential equation by substituting the derivatives for differential quotients. In the finite difference approach, by discretizing the continuous domain of the independent variables, we obtain a finite set of nodes in which the partial derivatives are replaced with and approximated by appropriate difference quotients. In this way, we can set up a difference algebraic equation at each mesh point and obtain a system of algebraic equations. These can be solved quite easily depending on the type of PDE problem, one at a time (explicit schemes) or simultaneously (implicit schemes). Most of the schemes constructed in our presentation are explicit, but resorting to an implicit scheme in some of the problems that we address is a suitable option.

The error between the numerical and the exact solution is evaluated by taking into account the deviation that incurs when replacing the differential operator with a difference operator. This error is known as the discretization error or truncation error, and indicates that a truncated series or Taylor polynomial is used in the approximation. This term is not known and its value is of order $\mathcal{O}\left(h^{n+1}\right)$, see [72]. From this point onwards, we consider smooth enough two arguments functions because, in the evolution problems that we treat, the unknowns are functions of two arguments $U(x, t)$, where $x$ and $t$ respectively stand for spatial and temporal variables.

Without loss of generality, let's consider a general two-dimensional domain $\{(x, t) ; 0 \leq x \leq L, 0 \leq t \leq T\}$ in which an evolution PDE problem is stated, where $L$ and $T$ respectively hold for the amplitude of the spatial region and the time horizon. Discretization is performed by partitioning the two domains with the introduction of a step size or discretization parameter ( $h$ for space, $k$ for time) that separates the points where the discrete problem is posed by taking into account the following relationships: $h=\Delta x=L / M ; k=\Delta t=T / N$, and $x_{j}=j h, t^{n}=n k$.

As it will be mentioned later when introducing the concept of consistency of a numerical scheme, for our purposes it is essential that as the separation between nodes or the step size becomes smaller, the numerical approximation to the exact solution becomes more accurate. Step sizes do not necessarily have to be constant 
throughout the interval, but in this work we consider that they are fixed for each independent variable. As a result, we obtain a discrete domain composed of two finite sets of indexed mesh points, $\left\{x_{j}, 0 \leq j \leq M\right\}$ and $\left\{t^{n}, 0 \leq n \leq N\right\}$, where $M$ and $N$ are positive integers in which numerical solution $u_{j}^{n}$ should approximate exact solution $U(j h, n k)$.

In principle, finite difference approximations can be formulated in an infinite number of forms depending on the stencil we choose for the point of interest; i.e., the number of neighbouring nodes taken into account, that determine the order of the approximation, and the geometric arrangement considered to build the difference quotient.

With the above-described mesh, we recall (see [72]) that the first-order spatial backward (left-sided) difference approximation of a function of two arguments $U(x, t)$ at the mesh point $\left(x_{j}, t^{n}\right)$ takes the form:

$$
\frac{\partial U\left(x_{j}, t^{n}\right)}{\partial x}=\frac{u_{j}^{n}-u_{j-1}^{n}}{h}+\mathcal{O}(h) .
$$

Similarly, it is easy to obtain some other most frequently used first-derivative approximations, and their corresponding order:

-First-order spatial forward (right-sided)

$$
\frac{\partial U\left(x_{j}, t^{n}\right)}{\partial x}=\frac{u_{j+1}^{n}-u_{j}^{n}}{h}+\mathcal{O}(h) .
$$

-Second-order spatial central

$$
\frac{\partial U\left(x_{j}, t^{n}\right)}{\partial x}=\frac{u_{j+1}^{n}-u_{j-1}^{n}}{2 h}+\mathcal{O}\left(h^{2}\right) .
$$

-Second-order spatial backward (left-sided)

$$
\frac{\partial U\left(x_{j}, t^{n}\right)}{\partial x}=\frac{3 u_{j}^{n}-4 u_{j-1}^{n}+u_{j-2}^{n}}{h}+\mathcal{O}\left(h^{2}\right) .
$$

-Second-order spatial forward (right-sided)

$$
\frac{\partial U\left(x_{j}, t^{n}\right)}{\partial x}=\frac{-3 u_{j}^{n}+4 u_{j+1}^{n}-u_{j+2}^{n}}{h}+\mathcal{O}\left(h^{2}\right) .
$$


-Fourth-order spatial central

$$
\frac{\partial U\left(x_{j}, t^{n}\right)}{\partial x}=\frac{-u_{j+2}^{n}+8 u_{j+1}^{n}-8 u_{j-1}^{n}+u_{j-2}^{n}}{12 h}+\mathcal{O}\left(h^{4}\right) .
$$

The commonest second-derivative approximations ones are given by:

-First-order spatial backward (left-sided)

$$
\frac{\partial U^{2}\left(x_{j}, t^{n}\right)}{\partial x^{2}}=\frac{u_{j}^{n}-2 u_{j-1}^{n}+u_{j-2}^{n}}{h^{2}}+\mathcal{O}(h) .
$$

-First-order spatial forward (right-sided)

$$
\frac{\partial U^{2}\left(x_{j}, t^{n}\right)}{\partial x^{2}}=\frac{u_{j}^{n}-2 u_{j+1}^{n}+u_{j+2}^{n}}{h^{2}}+\mathcal{O}(h) .
$$

-Second-order spatial central

$$
\frac{\partial U^{2}\left(x_{j}, t^{n}\right)}{\partial x^{2}}=\frac{u_{j+1}^{n}-2 u_{j}^{n}+u_{j-1}^{n}}{h^{2}}+\mathcal{O}\left(h^{2}\right) .
$$

-Fourth-order spatial central

$$
\frac{\partial U^{2}\left(x_{j}, t^{n}\right)}{\partial x^{2}}=\frac{-u_{j+2}^{n}+16 u_{j+1}^{n}-30 u_{j}^{n}+16 u_{j-1}^{n}-u_{j-2}^{n}}{12 h^{2}}+\mathcal{O}\left(h^{4}\right) .
$$

Once the mesh is constructed, we work with discrete variables by replacing the derivatives of the PDE problem (including those which appear in the initial, boundary and Stefan conditions, if any) with suitable finite difference approximations. At this point, we rearrange the discrete equations to obtain a numerical scheme that can be solved by its implementation as a finite difference algorithm in a computer.

Depending on how the temporal derivative is approximated, three basic schemes can be considered:

-Explicit scheme, in which approximation consists of a forward difference quotient of the type:

$$
\frac{\partial U\left(x_{j}, t^{n}\right)}{\partial t} \approx \frac{u_{j}^{n+1}-u_{j}^{n}}{k}
$$

evaluated at the mesh point $(j h, n k)$. One of the major advantages of explicit finite difference methods lies in their relative simplicity and short computation time. However, the main drawback is that explicit schemes are stable only under some 
conditions that link the size of the discretization steps, and the truncation error is first-order in time; i.e. $\mathcal{O}(k)$.

-Implicit scheme, in which the derivative is approximated by a backward difference quotient; i.e., a similar expression to (1.17), but calculated at the mesh point $(j h,(n+1) k)$. The implicit scheme is unconditionally stable regardless of the time step size, and is first order accurate in time; i.e., $\mathcal{O}(k)$.

-Crank-Nicolson scheme. This scheme considers the average approximation of the above two. The Crank-Nicolson scheme is unconditionally stable and secondorder accurate in time; i.e., $\mathcal{O}\left(k^{2}\right)$.

\subsubsection{Preliminary concepts of numerical analysis}

The following concepts are important for the analysis of the finite difference schemes related to evolution PDE problems. The reliability and computational efficiency of a finite difference method depends on whether the scheme is consistent, stable and convergent, or not.

Truncation error. Truncation errors arise when exact mathematical expressions are represented by approximations. In our case, the truncation error of the derivatives is the difference between their exact value and their finite-difference estimations by truncated Taylor series. With notation $\mathcal{O}(h)$, we mean that the absolute truncation error value is bounded by product $K h$ for $h$ small enough, where $K$ is a positive real constant and $h$ is the discretization step size. Generally, we state that $f(x)$ is of order $g(x)$, i.e. $f(x)=\mathcal{O}[g(x)]$, where $f(x)$ and $g(x)$ are real or complex functions if a positive constant $K$ exists that is independent of $x$, so that $|f(x)| \leq K|g(x)|$ for all $x$.

Roundoff error. Roundoff errors arise when using digital computers because of their limited capacity and precision to represent numbers, and also because they cannot often work with exact quantities. Some numerical operations (e.g., subtracting nearly equal digits) are highly sensitive to roundoff errors. The total numerical error is the sum of the truncation and the roundoff error.

Consistency. Consistency is a term linked to truncation error as it deals with how much the finite difference equations approximate the PDE in a particular problem. A finite difference scheme is stated to be consistent with a PDE if the trun- 


\subsection{Numerical treatment of moving boundary problems}

cation error vanishes as the step size tends to zero; i. e., as the mesh is refined. In this case, the discrete operator that defines the finite difference tends to be the equivalent to the analytical operator when discretization step sizes approach zero.

Stability. In addition to consistency, scheme stability is a necessary property. The roundoff errors incurred in calculations may lead to a disruption in the whole computation process. Roughly speaking, we can state that a numerical scheme is stable if roundoff errors are not amplified in calculations. Stability ensures that the error remains bounded as numerical computation progresses. As stability is related to the numerical solution's boundedness, it is necessary to specify a norm. We denote the so-called supremum norm of a vector $x=\left(x_{1}, x_{2}, \ldots, x_{n}\right)^{T}$ in $\mathbb{R}^{n}$ as $\|x\|_{\infty}=\max \left(\left|x_{1}\right|,\left|x_{2}\right|, \ldots,\left|x_{n}\right|\right)$.

Using the notation for the solution vector at time level $n, w^{n}=\left[w_{0}^{n}, w_{1}^{n}, \ldots, w_{M}^{n}\right]^{T}$, we state that numerical solution $\left\{w^{n}, \quad 0 \leq n \leq N\right\}$ is $\|\cdot\|_{\infty}$-stable if a positive constant $C$ exists and is independent of $n, k$ and $h$ so that:

$$
\left\|w^{n}\right\|_{\infty} \leq C, \quad 0 \leq n \leq N
$$

Convergence. Convergence means that the solution of a PDE problem and its associated finite difference equation come closer as the mesh is refined. In other words, the numerical solution converges towards the exact solution of the PDE for step sizes that approach zero. From Lax's equivalence theorem, it is known that a consistent scheme for well-posed linear evolution problems is convergent if it is stable. 



\section{Population dynamics of an invasive species}

As argued in Chapter 1, a moving boundary problem is characterized by the fact that the boundary of the domain is not known in advance but it has to be determined as a part of the solution. These problems are often called Stefan problems due to the Stefan condition that links the behaviour of the boundary with the unknown solution, see [26, 66]. The term free-boundary problem is commonly used when the boundary is independent of the time and typically related to elliptic problems. Moving boundary problems have their origins in physical and engineering problems [26, 31], and more recently in biological and physiological sciences [19], decision and control theory and ecology [30].

Prior to [30] the modelling of biological invasions has been widely studied in [4, 5, 10, 34, 43, 50, 71, 83, 84] under the crucial restriction that in the previous papers the spatial domain is not constrained by the population behavior, that is the essence of the Stefan condition. The first diffusive logistic model related to biological invasions was initiated in 1937, of course without boundary restrictions, independently by Fisher [34] and Kolmogorov-Petrovsky-Piskunov (KPP) [43]. Very recent papers have treated numerically these nonlinear models focusing 
on the stability and the preservation of the qualitative properties of the theoretical solution [11, 52, 63].

To our knowledge the seminal paper [30] by Du and Lin is the first contribution in the field of spreading of populations where a Stefan condition is used and managing a moving boundary problem of parabolic type. Further developments of this problem have been treated in [28, 29, 82]. The diffusive logistic model of [30] for the density of population of the invasive species $U(t, x)$ depending on time $t$ and spatial variable $x$ states as follows:

$$
\frac{\partial U}{\partial t}-D \frac{\partial^{2} U}{\partial x^{2}}=U(a-b U), \quad t>0,0<x<H(t),
$$

together with the boundary conditions

$$
\frac{\partial U}{\partial x}(t, 0)=0, \quad U(t, H(t))=0, \quad t>0
$$

the Stefan condition

$$
H^{\prime}(t)=-\mu \frac{\partial U}{\partial x}(t, H(t)), \quad t>0
$$

and the initial conditions

$$
H(0)=H_{0}, \quad U(0, x)=U_{0}(x), \quad 0 \leq x \leq H_{0}
$$

The initial function $U_{0}(x)$ satisfies the following properties:

$$
U_{0}(x) \in C^{2}\left(\left[0, H_{0}\right]\right), \quad U_{0}^{\prime}(0)=U_{0}\left(H_{0}\right)=0, \quad U_{0}(x)>0, \quad 0 \leq x<H_{0} .
$$

Here $H(t)$ is the unknown moving boundary such that the population is distributed in the interval $[0, H(t)], D>0$ is the dispersal rate and the positive parameters $a$ and $b$ are the intrinsic growth rate and the intraspecific competition, respectively. The parameter $\mu>0$ involved in the Stefan condition (2.3) is the proportionality constant between the population gradient at the front and the speed of the moving boundary. Unlike to the previous models, where only spreading behaviour was admissible, the authors of [30] show by the very first time a dichotomic alternative behavior, vanishing or spreading approach to the habitat carrying capacity $a / b$, depending on the initial front and population density and the value of the parameter 
$\mu$ appearing in Stefan condition. According to [30], there is a threshold $\mu^{*}$ whose value is not known in advance, splitting the vanishing-spreading behavior.

This chapter aims to be a continuation and numerical completion of [30] with the conviction that the best model may be wasted with a careless numerical treatment. Apart from the computation of the population density solution of problem (2.1)-(2.4) and the numerical analysis detailed below, this chapter has the potential advantage that allows us the computation of the expanding front of the species population as well as the approximation, by means of numerical experiments included in Section 2.4, of the crucial parameter $\mu^{*}$, whose existence is guaranteed in [30] but whose value is not known in terms of data problem. A brief numerical treatment of the problem may be found in Section 3.6 of [15].

Chapter 2 is organized as follows. In Section 2.1, and following the trajectory of the authors in the study of finance problems (see [21, 22]), we use the well-known Landau transformation (see [26, 49]), in order to convert the problem (2.1)-(2.4) into a fixed spatial domain one, where the moving boundary is included as another variable to solve apart from the population density. We also include in Section 2.1 the discretization of the transformed problem achieving an explicit finite difference scheme allowing the computation not only of the population but also of the expanding front. Section 2.2 deals with the study of the consistency of the scheme with the transformed problem. Dealing with population problems it is important to guarantee the positivity of the numerical solution; this qualitative property together with the stability of the numerical solution and the positivity and monotone behaviour of the numerical expanding front are studied in Section 2.3. Section 2.4 illustrates with numerical examples the dichotomic behaviour of the numerical solution of the problem. The chapter ends with some relevant conclusions in Section 2.5.

\subsection{Transformation and discretization of the continu- ous problem}

Let us begin this section by transforming the moving front problem (2.1)-(2.4) into a problem with a fixed domain $[0,1]$. Let us consider the Landau transformation, 
[26, 49],

$$
z(t, x)=\frac{x}{H(t)}, \quad W(t, z)=U(t, x) .
$$

Under substitution (2.6) problem (2.1)-(2.4) takes the form:

$$
G(t) \frac{\partial W}{\partial t}-G^{\prime}(t) \frac{z}{2} \frac{\partial W}{\partial z}-D \frac{\partial^{2} W}{\partial z^{2}}=G(t) W(a-b W), \quad t>0,0<z<1
$$

where:

$$
G(t)=H^{2}(t), \quad t \geq 0 .
$$

Boundary conditions (2.2) and Stefan condition (2.3) take the form:

$$
\frac{\partial W}{\partial z}(t, 0)=0, \quad W(t, 1)=0, \quad t>0
$$

and

$$
G^{\prime}(t)=-2 \mu \frac{\partial W}{\partial z}(t, 1), \quad t>0
$$

respectively, while the initial conditions (2.4) become:

$$
G(0)=H_{0}^{2}, \quad W(0, z)=W_{0}(z)=U_{0}\left(z H_{0}\right), \quad 0 \leq z \leq 1 .
$$

Conditions 2.5 for the initial function $U_{0}(x)$ are translated to $W_{0}(z)$ as follows:

$$
W_{0}(z) \in C^{2}([0,1]), \quad W_{0}^{\prime}(0)=W_{0}(1)=0, \quad W_{0}(z)>0, \quad 0 \leq z<1 .
$$

After the transformation, the new problem lies in solving the nonlinear parabolic partial differential equation 2.7) in the unbounded fixed domain $(0, \infty) \times$ $(0,1)$ for the variables $(t, z)$. Regarding the temporal interval, for numerical analysis reasons, it is considered here to be bounded, ranging from the initial time to a bounded time horizon, $T$. Let us consider the step size discretization $k=\Delta t=$ $T / N, h=\Delta z=1 / M$, and the mesh points $\left(t^{n}, z_{j}\right)$, with $t^{n}=k n, 0 \leq n \leq N$, $z_{j}=j h, 0 \leq j \leq M$ and $N, M$ positive integers. As agreed in Section 1.3, let us denote the approximate value of $W\left(t^{n}, z_{j}\right)$ at the mesh point $\left(t^{n}, z_{j}\right)$,

$$
w_{j}^{n} \approx W\left(t^{n}, z_{j}\right)
$$

and let $g^{n}$ be the approximation of $G\left(t^{n}\right)$. 


\subsection{Transformation and discretization of the continuous problem}

On the assumption that functions $W(t, z)$ and $G(t)$ satisfy the requirement $W \in$ $C^{1,2}(\Omega)$, where $\Omega=\left\{(t, z) \in \mathbb{R}^{2}: t \in[0, T], z \in[0,1]\right\}$, and $G \in C^{1}[0, T]$, let us consider the forward approximation of the time derivatives,

$$
\frac{w_{j}^{n+1}-w_{j}^{n}}{k} \approx \frac{\partial W}{\partial t}\left(t^{n}, z_{j}\right), \quad \frac{g^{n+1}-g^{n}}{k} \approx G^{\prime}\left(t^{n}\right),
$$

and the central approximation of the spatial derivatives,

$$
\frac{w_{j+1}^{n}-w_{j-1}^{n}}{2 h} \approx \frac{\partial W}{\partial z}\left(t^{n}, z_{j}\right), \quad \frac{w_{j-1}^{n}-2 w_{j}^{n}+w_{j+1}^{n}}{h^{2}} \approx \frac{\partial^{2} W}{\partial z^{2}}\left(t^{n}, z_{j}\right) .
$$

From (2.14) and (2.15) the equation (2.7) is approximated by:

$$
\begin{aligned}
g^{n} \frac{w_{j}^{n+1}-w_{j}^{n}}{k}- & \frac{z_{j}}{2} \frac{w_{j+1}^{n}-w_{j-1}^{n}}{2 h}\left(\frac{g^{n+1}-g^{n}}{k}\right)-D \frac{w_{j-1}^{n}-2 w_{j}^{n}+w_{j+1}^{n}}{h^{2}} \\
& =g^{n} w_{j}^{n}\left(a-b w_{j}^{n}\right), \quad 0 \leq n \leq N-1, \quad 0 \leq j \leq M-1,
\end{aligned}
$$

that can be written as:

$$
\begin{aligned}
& w_{j}^{n+1}=\left[\frac{D k}{h^{2} g^{n}}-\frac{z_{j}}{4 h}\left(\frac{g^{n+1}}{g^{n}}-1\right)\right] w_{j-1}^{n}+\left[1+k\left(a-b w_{j}^{n}\right)-\frac{2 D k}{h^{2} g^{n}}\right] w_{j}^{n} \\
& +\left[\frac{D k}{h^{2} g^{n}}+\frac{z_{j}}{4 h}\left(\frac{g^{n+1}}{g^{n}}-1\right)\right] w_{j+1}^{n}, \quad 0 \leq n \leq N-1, \quad 0 \leq j \leq M-1 .
\end{aligned}
$$

As it is usual in numerics we assume that equation (2.7) can be also approximated at $z_{j}=0$. Equation (2.17) written for $j=0$ involves the fictitious value $w_{-1}^{n}$ at the point $\left(t^{n},-h\right)$. This value $w_{-1}^{n}$ is eliminated from the discretization of the boundary and initial conditions (2.9) and (2.11),

$$
\frac{w_{1}^{n}-w_{-1}^{n}}{2 h}=0, \quad w_{M}^{n}=0, \quad 0 \leq n \leq N .
$$

Transformed Stefan condition 2.10 is discretized using first order forward approximation for $G^{\prime}(t)$ and three points backward spatial approximation of $\frac{\partial W}{\partial z}(t, 1)$ :

$$
\frac{g^{n+1}-g^{n}}{k}=-\frac{\mu}{h}\left(3 w_{M}^{n}-4 w_{M-1}^{n}+w_{M-2}^{n}\right), \quad 0 \leq n \leq N-1,
$$

to preserve accuracy of order $\mathcal{O}(k)+\mathcal{O}\left(h^{2}\right)$. From 2.18 equation 2.19 can be rewritten as:

$$
g^{n+1}=g^{n}+\frac{k \mu}{h}\left(4 w_{M-1}^{n}-w_{M-2}^{n}\right), \quad 0 \leq n \leq N-1 .
$$


Finally, replacing (2.20) in the explicit scheme 2.17), we have:

$$
w_{j}^{n+1}=a_{j}^{n} w_{j-1}^{n}+b_{j}^{n} w_{j}^{n}+c_{j}^{n} w_{j+1}^{n}, \quad 0 \leq n \leq N-1, \quad 0 \leq j \leq M-1,
$$

where the coefficients are given by:

$$
\begin{array}{r}
a_{j}^{n}=\frac{k}{h^{2}}\left(\frac{D}{g^{n}}-\frac{z_{j} \mu\left(4 w_{M-1}^{n}-w_{M-2}^{n}\right)}{4 g^{n}}\right), \\
b_{j}^{n}=1+k\left(a-b w_{j}^{n}\right)-\frac{k}{h^{2}} \frac{2 D}{g^{n}}, \\
c_{j}^{n}=\frac{k}{h^{2}}\left(\frac{D}{g^{n}}+\frac{z_{j} \mu\left(4 w_{M-1}^{n}-w_{M-2}^{n}\right)}{4 g^{n}}\right), \\
n \geq 0, \quad 0 \leq j \leq M-1 .
\end{array}
$$

\subsection{Consistency}

Consistency of a numerical scheme with a PDE problem means that the theoretical solution of the problem approximates well the numerical scheme when the step size discretizations tend to zero. So, a numerical scheme can be consistent with an equation and not with another one, see [72], Chapter 2. Thus, it is important to address the consistency of a numerical scheme with a problem.

Let us consider the problem 2.7 -2.11, denoted in vector form as $\mathcal{L}(W, G)=$ $\left(\mathcal{L}_{1}(W, G), \mathcal{L}_{2}(W, G), \mathcal{L}_{3}(W, G)\right)$ where equations 2.7), 2.9), 2.10) are written in the form:

$$
\begin{gathered}
\mathcal{L}_{1}(W, G)=\frac{\partial W}{\partial t}-\frac{G^{\prime}(t)}{G(t)} \frac{z}{2} \frac{\partial W}{\partial z}-\frac{D}{G(t)} \frac{\partial^{2} W}{\partial z^{2}}-W(a-b W)=0, \quad t>0,0<z<1 \\
\mathcal{L}_{2}(W, G)=\frac{\partial W}{\partial z}(t, 0)=0, \quad t>0 \\
\mathcal{L}_{3}(W, G)=G^{\prime}(t)+2 \mu \frac{\partial W}{\partial z}(t, 1)=0, \quad t>0
\end{gathered}
$$

and the finite difference scheme 2.16, 2.18, 2.20, written together as $L(w, g)=$ $\left(L_{1}(w, g), L_{2}(w, g), L_{3}(w, g)\right)$ where:

$$
\begin{aligned}
& L_{1}(w, g)=\frac{w_{j}^{n+1}-w_{j}^{n}}{k}-\frac{z_{j}}{2} \frac{w_{j+1}^{n}-w_{j-1}^{n}}{2 h}\left(\frac{g^{n+1}-g^{n}}{g^{n} k}\right)-w_{j}^{n}\left(a-b w_{j}^{n}\right) \\
& -\frac{D}{g^{n}} \frac{w_{j-1}^{n}-2 w_{j}^{n}+w_{j+1}^{n}}{h^{2}}=0, \quad 0 \leq n \leq N-1, \quad 0 \leq j \leq M-1,
\end{aligned}
$$




$$
\begin{gathered}
L_{2}(w, g)=\frac{w_{1}^{n}-w_{-1}^{n}}{2 h}=0,0 \leq n \leq N, \\
L_{3}(w, g)=\frac{g^{n+1}-g^{n}}{k}-\frac{\mu}{h}\left(4 w_{M-1}^{n}-w_{M-2}^{n}\right)=0, \quad 0 \leq n \leq N-1 .
\end{gathered}
$$

In accordance with [72], scheme $L(w, g)$ is said to be consistent with problem $\mathcal{L}(W, G)$ if local truncation error $T_{j}^{n}(W, G)=\left(T(1)_{j}^{n}, T(2)_{j}^{n}, T(3)_{j}^{n}\right)$,

$$
\begin{aligned}
& T(1)_{j}^{n}(W, G)=L_{1}\left(W_{j}^{n}, G^{n}\right)-\mathcal{L}_{1}\left(W_{j}^{n}, G^{n}\right), \\
& T(2)_{j}^{n}(W, G)=L_{2}\left(W_{n}^{j}, G^{n}\right)-\mathcal{L}_{2}\left(W_{j}^{n}, G^{n}\right), \\
& T(3)_{j}^{n}(W, G)=L_{3}\left(W_{j}^{n}, G^{n}\right)-\mathcal{L}_{3}\left(W_{j}^{n}, G^{n}\right),
\end{aligned}
$$

tend to zero as $k \rightarrow 0, h \rightarrow 0$, where $W_{j}^{n}=W\left(t^{n}, z_{j}\right)$ and $G^{n}=G\left(t^{n}\right)$ are the values of the exact solution of problem (2.7)-2.11) of both the PDE and the free boundary respectively at the point $\left(t^{n}, z_{j}\right)$. Now let us consider the local truncation error $T(1)_{j}^{n}$ assuming that the exact solution $W(t, z)$ satisfies $W \in C^{2,4}(\Omega)$. We also assume that $G(t)$ is two times continuously differentiable. By using Taylor's expansion about $\left(t^{n}, z_{j}\right)$ one gets:

$$
\begin{array}{r}
T(1)_{j}^{n}(W, G)=E_{j}^{n}(1) k-\frac{z_{j}}{2 G^{n}} G^{\prime}\left(t^{n}\right) E_{j}^{n}(3) h^{2}-\frac{z_{j}}{2 G^{n}} E^{n}(2) E_{j}^{n}(3) k h^{2} \\
-\frac{z_{j}}{2 G^{n}} \frac{\partial W}{\partial z}\left(t^{n}, z_{j}\right) E^{n}(2) k-\frac{D}{G^{n}} E_{j}^{n}(4) h^{2},
\end{array}
$$

where:

$$
\begin{gathered}
E_{j}^{n}(1)=\frac{1}{2} \frac{\partial^{2} W}{\partial t^{2}}\left(\tau, z_{j}\right), \quad t^{n}<\tau<t^{n+1} . \\
E^{n}(2)=\frac{1}{2} \frac{d^{2} G}{d t^{2}}(\delta), \quad t^{n}<\delta<t^{n+1} . \\
E_{j}^{n}(3)=\frac{1}{6} \frac{\partial^{3} W}{\partial z^{3}}\left(t^{n}, \xi_{1}\right), \quad z_{j-1}<\xi_{1}<z_{j+1} . \\
E_{j}^{n}(4)=\frac{1}{12} \frac{\partial^{4} W}{\partial z^{4}}\left(t^{n}, \xi_{2}\right), \quad z_{j-1}<\xi_{2}<z_{j+1} .
\end{gathered}
$$

Hence, the local truncation error satisfies:

$$
T(1)_{j}^{n}(W, G)=\mathcal{O}(k)+\mathcal{O}\left(h^{2}\right) .
$$

From 2.24, 2.27) and 2.30 one gets that $T(2)_{j}^{n}(W, G)=\mathcal{O}\left(h^{2}\right)$ while from 2.25, 2.28 and 2.31 it follows that $T(3)_{j}^{n}(W, G)=\mathcal{O}(k)+\mathcal{O}\left(h^{2}\right)$. Summarizing the following result has been established: 
Theorem 1. With previous notation, the scheme $L(w, g)$ is consistent with the problem $\mathcal{L}(W, G)$ and the local truncation error behaves as:

$$
T_{j}^{n}(W, G)=\mathcal{O}(k)+\mathcal{O}\left(h^{2}\right)
$$

\subsection{Positivity and Stability}

\subsubsection{Positivity}

Dealing with population models it is necessary to guarantee that the numerical solution is nonnegative. In this section we show that the numerical solution of the scheme (2.21)-(2.22) is nonnegative for small enough values of the step size discretization. We also prove that the numerical solution preserves qualitative properties of the exact unique classical solution of the problem obtained by Du and Lin in [30].

We prove the nonnegativity of the solution $w_{j}^{n}$ of $2.21-2.22$ as well as the positivity and monotonicity of the free boundary $g^{n}$ using the induction principle on the index $n$. For $n=0$, from the initial conditions $2.12 w_{j}^{0}>0,0 \leq j \leq M-1$ and particularly $w_{M-1}^{0}>0$. From (2.12) we also have that the left hand side derivative $W_{0}^{\prime}\left(1^{-}\right)$at $z=1$ and hence the corresponding difference approximation $\left(3 w_{M}^{0}-4 w_{M-1}^{0}+w_{M-2}^{0}\right) /(2 h)=\left(w_{M-2}^{0}-4 w_{M-1}^{0}\right) /(2 h)<0$ for small enough values of $h$. As $g^{0}>0$, from 2.19, one gets:

$$
g^{1}>g^{0}>0
$$

Let us suppose that $w_{j}^{l}>0$ and $g^{l}>g^{l-1}>\ldots>g^{0}>0, \quad 1 \leq l \leq n$. We will prove that $w_{j}^{n+1}>0$ and $g^{n+1}>g^{n}$. By using Taylor's expansion on the left about $z_{M}=1$ one gets:

$$
w_{M-2}^{n}=2 w_{M-1}^{n}+\mathcal{O}\left(h^{2}\right), \quad 0 \leq n \leq N
$$

Note that from 2.20, 2.40 and using that $w_{M}^{n}=0$ and $w_{M-1}^{n}=O(h)$, one gets:

$$
g^{n+1}=g^{n}+\frac{k \mu}{h}\left(4 w_{M-1}^{n}-w_{M-2}^{n}\right)=g^{n}+\frac{k \mu}{h}\left(2 w_{M-1}^{n}+\mathcal{O}\left(h^{2}\right)\right)=g^{n}+\mathcal{O}(k),
$$


as it is expected from the differentiability of function $g(t)$ (cf. [30]).

Coming back to the positivity issues, let us consider the equation 2.21) for $j=$ $M-1$. From (2.22) and (2.40) one gets:

$$
w_{M-1}^{n+1}=\left(1+k\left(a-b w_{M-1}^{n}\right)-\frac{k}{h^{2}} \frac{z_{M-1}}{g^{n}} \mu w_{M-1}^{n}\right) w_{M-1}^{n}+\mathcal{O}\left(h^{2}\right) .
$$

As $g^{n}>g^{0}$ from the hypothesis and $z_{M-1}<1$, from 2.42 we can write:

$$
w_{M-1}^{n+1}>\left(1+k\left(a-b w_{M-1}^{n}\right)-\frac{k}{h^{2}} \frac{1}{g^{0}} \mu w_{M-1}^{n}\right) w_{M-1}^{n}=\varphi_{M-1}^{n} w_{M-1}^{n},
$$

for small enough values of $h$. As we are interested in showing that $\varphi_{M-1}^{n}>0$, let us start bounding $w_{M-1}^{n}$. From the expression of (2.42), for small enough values of $h$ we have that $w_{M-1}^{l+1}<w_{M-1}^{l}(1+k a), \quad 0 \leq l \leq n-1$. Recursively one gets:

$$
w_{M-1}^{l+1}<w_{M-1}^{0}(1+k a)^{l} \leq e^{a T} w_{M-1}^{0}, \quad 0 \leq l \leq n \leq N-1 ; \quad k N=T,
$$

for a time reference $T>0$.

From 2.44 and the definition of $\varphi_{M-1}^{n}$ given in 2.43) it is easy to show that $w_{M-1}^{n+1}>0$ under the condition:

$$
k<\frac{h^{2}}{\frac{\mu C}{g^{0}}+h^{2}(b C-a)},
$$

where

$$
C=e^{a T} w_{M-1}^{0} .
$$

Once the positivity of $w_{M-1}^{n}$ is established, it is necessary to show that $w_{j}^{n}>0$ for $0 \leq j \leq M-2$. From (2.21) and the induction hypothesis, this occurs when coefficients of the scheme are nonnegative. Note that from (2.22) and (2.40) every coefficient $c_{j}^{n}>0$ for small enough values of $h$. From 2.22, and taking into account that $0 \leq z_{j}<1$, coefficient $a_{j}^{n}>0$ if $\mu w_{M-1}^{n}<2 D$, and thus from 2.46 one concludes that $a_{j}^{n}>0$ holds true under the condition:

$$
w_{M-1}^{n}<\frac{2 D e^{-a T}}{\mu} .
$$

Let $B(n)$ be defined by $B(n)=\max \left\{w_{j}^{n} ;, 0 \leq j \leq M\right\}$. Using that $g^{n}>g^{0}>0$ by induction hypothesis $b_{j}^{n}>0$ if

$$
k<\frac{h^{2}}{\frac{2 D}{g^{0}}+h^{2}(b B(n)-a)} .
$$


In order to get an explicit expression of $B(n)$ independent of the discretization, note that from positivity of coefficients $a_{j}^{n}, b_{j}^{n}, c_{j}^{n}$ and (2.21):

$$
\begin{array}{r}
w_{j}^{n+1} \leq\left(1+k\left(a-b w_{j}^{n}\right)\right) B(n) \leq(1+k a) B(n) \leq(1+k a)^{2} B(n-1) \\
\leq \ldots \leq(1+k a)^{n} B(0)<e^{a T} B(0),
\end{array}
$$

where $B(0)=\max \{W(0, z)\}, \quad 0 \leq z \leq 1$. In order to prove the monotonicity of the free boundary $g^{n}$, from 2.40) and 2.20) one gets that $g^{n+1}>g^{n}$.

Summarizing, the following results have been established:

Theorem 2. With previous notation, let $k_{0}$ be:

$$
k_{0}=\min \left\{k_{1}=\frac{h^{2}}{\frac{\mu C}{g^{0}}+h^{2}(b C-a)}, k_{2}=\frac{h^{2}}{\frac{2 D}{g^{0}}+h^{2}\left(b e^{a T} B(0)-a\right)}\right\} .
$$

Under condition $k<k_{0}$ for small enough values of $h$ the solution $\left\{w_{j}^{n}, g^{n}\right\}$ of scheme (2.18), (2.20) and (2.21) verifies that $g^{n}$ is positive monotone increasing and:

$$
0 \leq w_{j}^{n} \leq B(0) e^{a T} ; \quad 0 \leq j \leq M, \quad 0 \leq n \leq N, \quad N k=T
$$

\subsubsection{Stability}

The concept of stability is somewhat plural in the literature. For the sake of clarity in the presentation, we specify the concept of stability we use below (see page 92 of [23, 44]), applicable by virtue of the smoothness of the solution. We recall the definition of the supremum norm of a vector $x=\left(x_{1}, x_{2}, \ldots, x_{n}\right)^{T}$ in $\mathbb{R}^{n}$ as $\|x\|_{\infty}=\max \left(\left|x_{1}\right|,\left|x_{2}\right|, \ldots,\left|x_{n}\right|\right)$.

Definition 1. Following the definition outlined in (1.18), the numerical scheme (2.19)-(2.21) is said to be $\|\cdot\|_{\infty}$-stable in the domain $[0, T] \times[0,1]$, if for every partition with $k=\Delta t, h=\Delta z, N k=T$ and $M h=1$ it holds true that:

$$
\left\|w^{n}\right\|_{\infty} \leq K\left\|w^{0}\right\|_{\infty}, \quad 0 \leq n \leq N
$$

where $w^{n}=\left[w_{0}^{n}, w_{1}^{n}, \ldots, w_{M}^{n}\right]^{T}$ is the vector solution of the scheme and $K$ is independent of $h, k$, and $n$. 
From 2.51, using that $B(n)=\left\|w^{n}\right\|_{\infty}$ and from Theorem 2 one gets $\left\|w^{n}\right\|_{\infty} \leq$ $K\left\|w^{0}\right\|_{\infty}$ with $K=e^{a T}$. Thus the following result has been established:

Theorem 3. With previous notation, under the condition $k<k_{0}$ where $k_{0}$ is given by (2.50) and small enough values of $h$ the numerical scheme (2.19)-(2.21) is conditionally $\|\cdot\|_{\infty}$-stable in the domain $[0, T] \times[0,1]$.

In the following examples, we show that the stability and positivity condition of Theorems 2 and 5 can not be disregarded and that in fact this is a tight condition. In Example 1 the condition is satisfied, however in Example 2 the stability and positivity condition is broken and results become unstable.

Example 1. Consider the logistic diffusion model (2.1)-2.4 with parameters $\left(D, \mu, a, b, H_{0}\right)=(5,5,5,1,2)$ and $U_{0}=\cos (\pi x / 4)$. For $h=0.05$ one gets $k_{1}=$ 0.0076 and $k_{2}=0.001$. Taking $k=0.00091$ stability is guaranteed as can be seen in Figure 2.1.

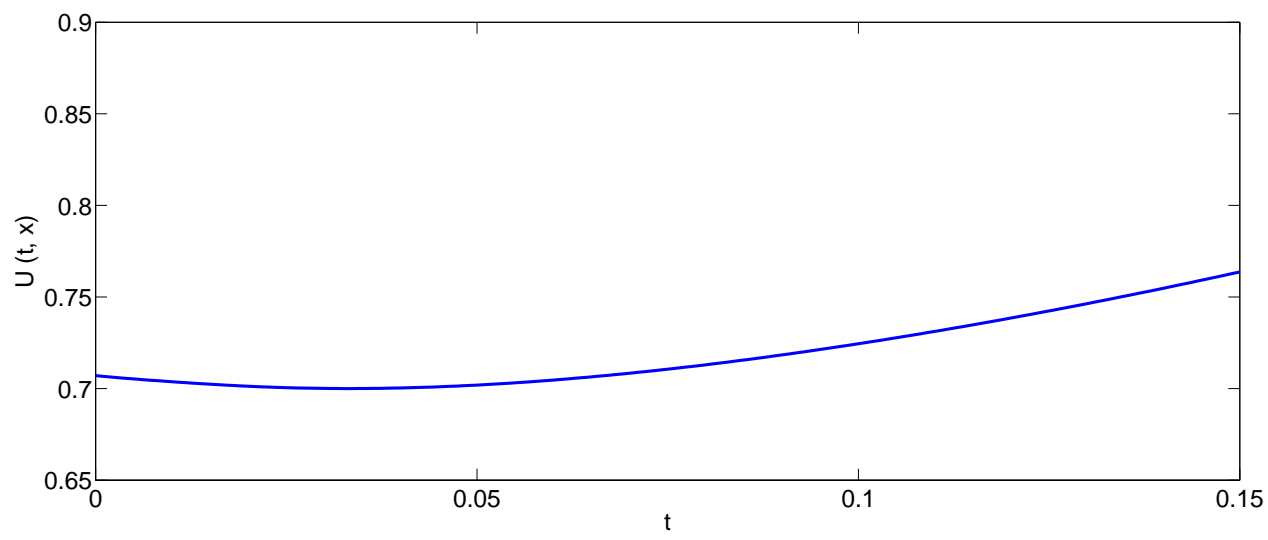

Figure 2.1: Numerical solution of Example 1 for $z=0.5$ under stability condition Numerical solution of Example 1 for $z=0.5$ under stability condition.

Example 2. With the same parameters and value of $h=0.05$ as in Example 1 , with $k=0.001179$, the stability condition is broken because $k>k_{2}$ and Figure 2.2 shows unstable results. 


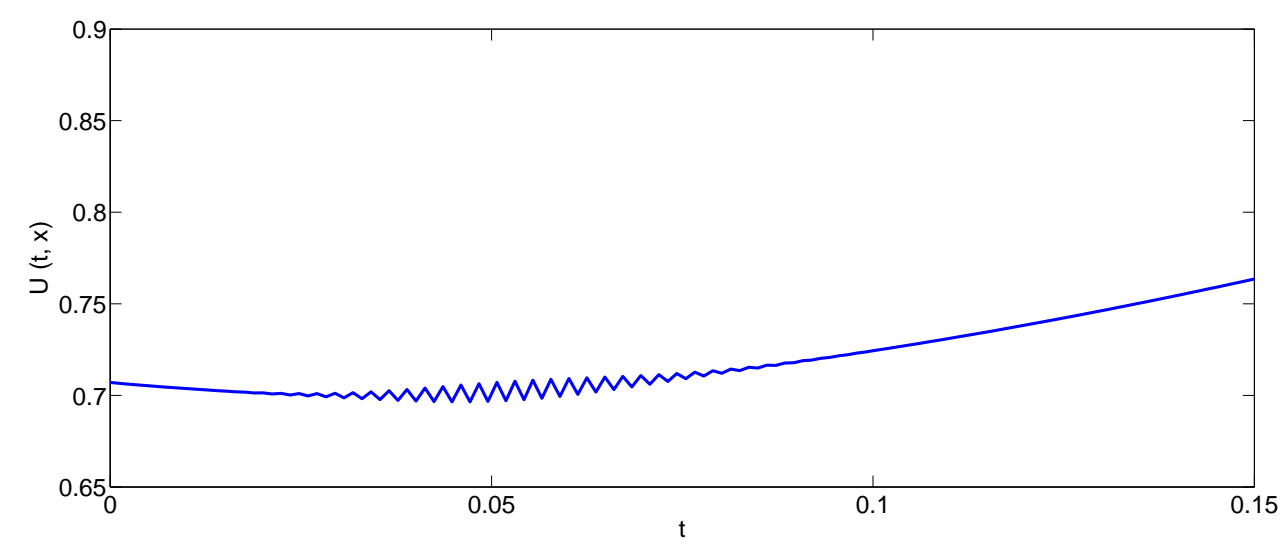

Figure 2.2: Numerical solution of Example 2 for $z=0.5$ when the stability condition is broken.

\subsection{Numerical dichotomy: spreading versus vanish- ing}

Theoretical results in [30] establish that for $H_{0} \geq L$, where $L=\frac{\pi}{2} \sqrt{\frac{D}{a}}$, spreading of the species is guaranteed. Even if $H_{0}<L$, spreading occurs under condition $\mu>\mu^{*}$ where $\mu^{*}$ is an unknown threshold depending on $U_{0}$, see Theorem 3.9 of [30]. In the spreading case the population density tends to the habitat carrying capacity limit $a / b$ as time tends to infinity, see Lemma 3.2 of [30]. For $H_{0}<L$ and $\mu \leq \mu^{*}$ vanishing happens, satisfying that $L$ is an upper bound of $H(t)$, i.e., $H(t) \leq L, \quad t>0$. The following example is devoted to spreading case showing that the numerical solution of problem (2.7)-2.11) computed by the proposed scheme 2.21)-2.22 converges to $a / b$ confirming that the numerical spreading occurs.

Example 3. Concerning the logistic diffusion model (2.1)-(2.4) with parameters values $\left(D, \mu, a, b, H_{0}\right)=(1,1,2,1,4)$ and $U_{0}=\cos (\pi x / 8)$, Figure 2.3 shows the spreading behaviour under condition $H_{0}=4.00>L=1.11$. Note that as time increases the numerical solution approaches to the habitat carrying capacity $a / b$. 


\subsection{Numerical dichotomy: spreading versus vanishing}

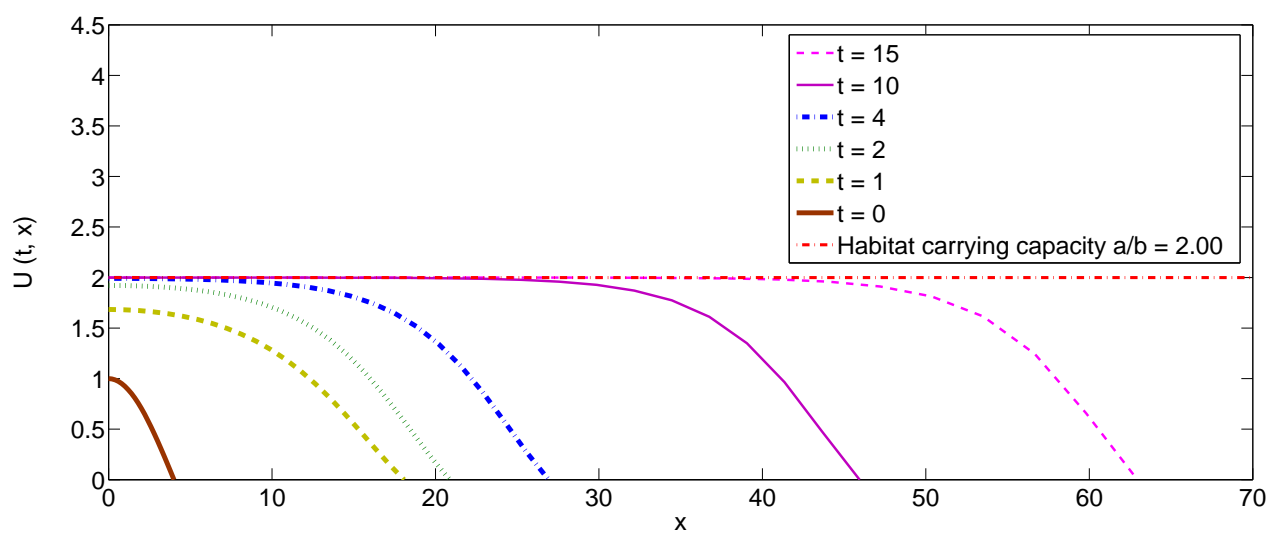

Figure 2.3: Numerical solution of Example 3 for several values of time in a spreading case.

The next example illustrates the vanishing behaviour of the numerical solution according to the theoretical results of [30].

Example 4. In this example we take $\left(D, \mu, a, b, H_{0}\right)=(0.1,0.2,0.04,0.04,1)$, with $U_{0}=\cos (\pi x / 2)$. There is vanishing behaviour with $H_{0}=1.00<L=2.48$ and $\mu=0.20$. Figure 2.4 shows that numerical population density tends to zero and the free boundary is always upper bounded by $L$. Besides, Table 2.1 exhibits CPU time for several values of the time horizon $T$ considered in the simulation.

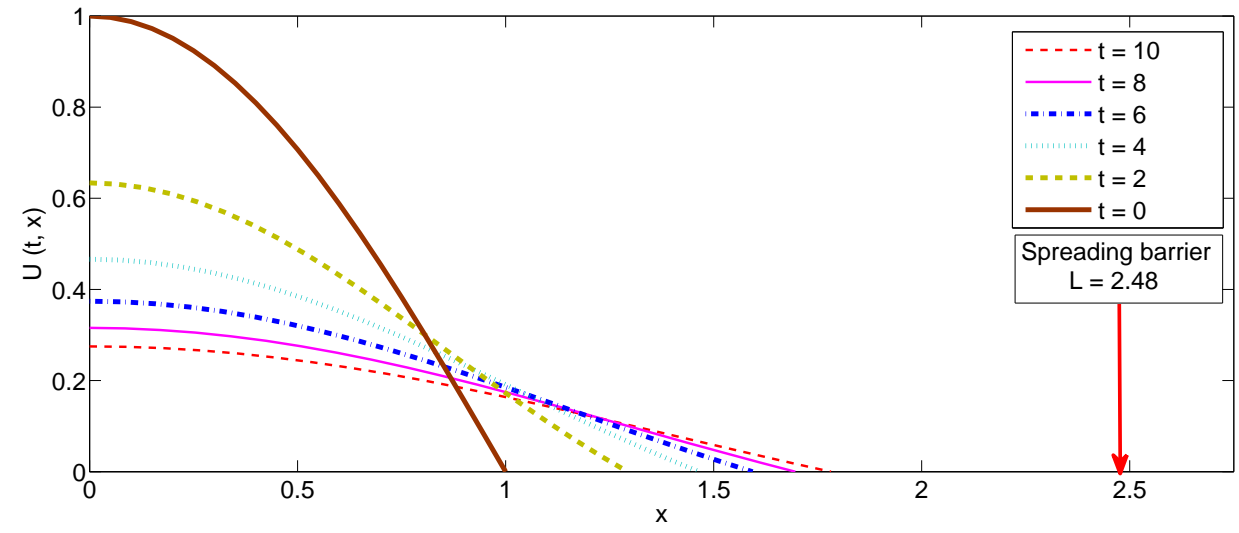

Figure 2.4: Numerical solution of Example 4 for several values of time in a vanishing case. 


\begin{tabular}{ll}
\hline \hline$T$ (years) & $\begin{array}{l}\text { CPU } \\
\text { (seconds) }\end{array}$ \\
\hline 2 & 0.186687 \\
4 & 0.490169 \\
6 & 1.097099 \\
8 & 6.850214 \\
10 & 31.021795 \\
\hline \hline
\end{tabular}

Table 2.1: Associated CPU time for several values of $T$ (Example 4).

One of the advantages of the proposed front-fixing numerical approach is to forecast the magnitude of the parameter $\mu^{*}$ whose existence is guaranteed in the theory but whose value is not known. In addition, the numerical solution of the free boundary $H\left(t^{n}\right)$ is obtained explicitly by expression (2.20), noting that $H\left(t^{n}\right)=$ $\sqrt{g^{n}}$

Next examples show the evolution of the free boundary (Example 5) and the speed of spreading behaviour (Example 6), taking into account that the forecasted value of the parameter $\mu^{*}$ is the threshold where the solution transits from vanishing to spreading.

Example 5. Choosing the values $\left(D, \mu, a, b, H_{0}\right)=(1, \mu, 1,1,1)$ and $U_{0}=$ $\cos (\pi x / 2)$, the evolution of the expanding front $H(t)$ for different values of $\mu$ is shown in Figure 2.5. The parameter $\mu^{*}$ which separates spreading from vanishing behaviour is estimated. In the vanishing cases, it can be seen that the "spreading barrier" is an upper bound for the expanding front as the theoretical results predict. 


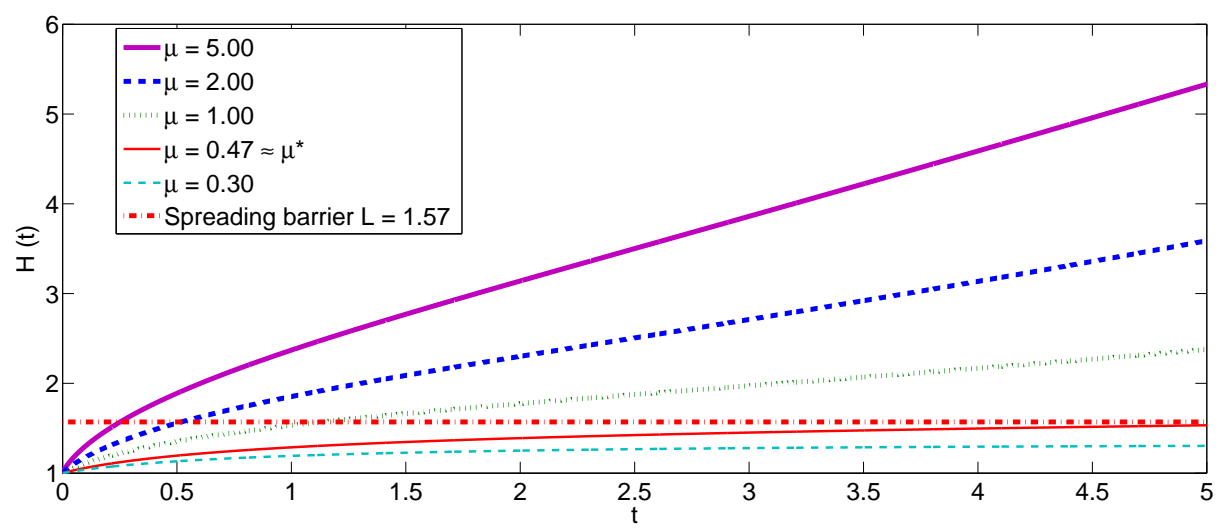

Figure 2.5: Expanding front $H(t)$ of Example 5 for several values of $\mu$.

Example 6. With the same values of the previous example, the speed of the front $d H / d t$ is illustrated in Figure 2.6. In the long term, for the spreading cases, the front speed tends to a nonzero constant value in accordance with [30], section 4, while in the vanishing case it tends to zero.

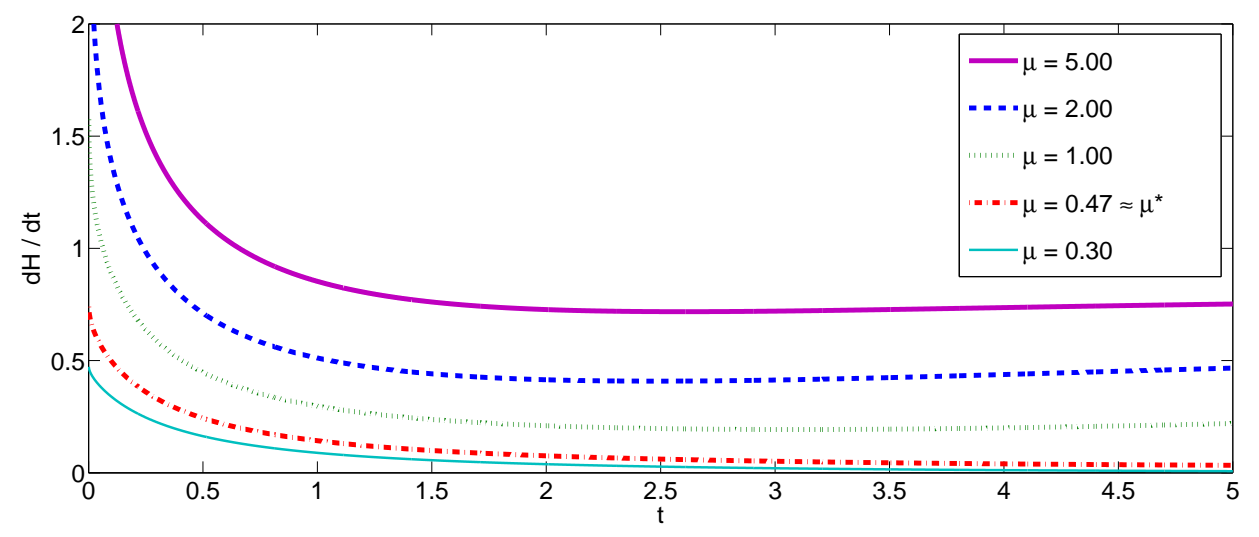

Figure 2.6: Expanding front speed $d H / d t$ of Example 6 for several values of $\mu$.

\subsection{Conclusions}

In this chapter, a front-fixing approach is introduced in such a way that the expanding front becomes a new unknown variable of the transformed problem. It has the advantage of achieving a fixed numerical domain and the availability of computing explicitly the expanding front as well as the approximation of the parameter $\mu *$, 
whose existence is guaranteed in [30] but its value is not known. We provide a careful numerical analysis of theoretical results given in [30]. Results and techniques are potentially applicable to problems in higher dimensions proposed in [28], or to the presence of two fronts in one dimension [30]. 


\section{2 species model for damage by carbonation of reinforced concrete structures}

The carbonation of concrete is a natural phenomenon that affects the performance, serviceability and safety of concrete structures, such as bridges, sewage pipes and seawalls. The diffusion of the environmental carbon dioxide in the dry parts and its reaction in the unsaturated concrete pores, cause a drop in $p H$ of the alkaline components near the steel bars, and the oxide film around the steel surface declines its ability to protect bars from corrosion. Gradually, the process penetrates deeper into de concrete shaping a carbonation front that separates the carbonated zone from the uncarbonated one. Thus, the carbonation process leads to the corrosion of the embedded reinforcing bars, reducing the service life of concrete structures. A good understanding of the evolution of the carbonation process is crucial to predict the life service of concrete structures and save important amounts of money and energy.

The bulk of these changes leads to damaging and destabilization of the concrete itself or of the reinforcement embedded in the concrete. It is well known that in all carbonation scenarios, gaseous carbon dioxide is assumed to be supplied from an 


\section{2 SPECIES MODEL FOR DAMAGE BY CARBONATION OF REINFORCED CONCRETE STRUCTURES}

inexhaustible exterior source to the concrete sample, [2, 41]. Carbon dioxide entering the non-saturated concrete sample through the air parts of the pores dissolves into the pore water and forms carbonic acid. This phenomenon, called concrete carbonation, may reduce the durability of reinforced concrete structures, causing the corrosion of the steel bars. The concrete carbonation level is measured throughout the $\mathrm{CO}_{2}$ mass concentration in air and water phases in the concrete pores, that needs to be calculated.

Empirical evidences of the behaviour of the carbonation front propagation have shown a dependence on time following the so-called $\sqrt{t}$-law, [13, 14, 37, 42, 61, 67, 70, 80]. In the framework of moving-boundary problems, to our knowledge, Tuutti [79] in 1982, was the first appealing to the square root of $t$-law in the problem of concrete carbonation. Such conclusions were based on the Neumann solution of the two-phase Stefan problem, see Section 13.2.2 of [26].

In recent papers [2, 3], the authors studied a one-dimensional free-boundary problem modeling the carbonation process. The unknown $\mathrm{CO}_{2}$ mass concentrations in air and water phases of pores are denoted by $U(t, x)$ and $V(t, x)$ respectively, depending on variables time $t$ and space $x$. The space variable $x$ is measured from the exposed boundary $x=0$ to the unknown carbonation front $x=S(t)$. In the system (3.2)-3.9) it is assumed that $\kappa_{1}$ and $\kappa_{2}$ are positive diffusion constants $\left(\kappa_{1} \gg \kappa_{2}\right)$ and the functions $f(U, V)$ and $\psi(r)$ are defined as

$$
f(U, V)=\beta(\gamma V-U), \beta>0, \gamma>0 .
$$

The continuous model is described by

$$
\begin{gathered}
\frac{\partial U}{\partial t}-\frac{\partial}{\partial x}\left(\kappa_{1} \frac{\partial U}{\partial x}\right)=f(U, V), \quad 0<t<T, \quad 0<x<S(t), \\
\frac{\partial V}{\partial t}-\frac{\partial}{\partial x}\left(\kappa_{2} \frac{\partial V}{\partial x}\right)=-f(U, V), \quad 0<t<T, \quad 0<x<S(t),
\end{gathered}
$$

together with the left boundary conditions

$$
U(t, 0)=G(t), \quad V(t, 0)=H(t), \quad 0 \leq t \leq T .
$$

The propagation front behaviour comes out from the Stefan-like conditions, involving function $\psi(r)$ linked to the chemical reactions:

$$
S^{\prime}(t)=\psi(U(t, S(t))), \quad 0<t<T,
$$




$$
\begin{gathered}
-\kappa_{1} \frac{\partial U}{\partial x}(t, S(t))=\psi(U(t, S(t)))+S^{\prime}(t) U(t, S(t)), \quad 0<t<T, \\
-\kappa_{2} \frac{\partial V}{\partial x}(t, S(t))=S^{\prime}(t) V(t, S(t)), \quad 0<t<T .
\end{gathered}
$$

Function $\psi(r)$ is given by

$$
\psi(r)=\alpha|r|^{p}, \quad r \in \mathbb{R}, \quad \alpha>0, \quad p \geq 1,
$$

where $p$ is the so-called the order of the chemical reaction.

The bounded initial conditions functions are described by

$$
S(0)=S_{0}, \quad U(0, x)=U_{0}(x), \quad V(0, x)=V_{0}(x), \quad 0<x<S_{0},
$$

where the initial concentrations satisfy the requirement $U_{0} \in C\left[0, S_{0}\right], V_{0} \in C\left[0, S_{0}\right]$.

Aiki and Muntean [2, 3] show qualitative properties of the solutions $U(t, x)$ and $V(t, x)$ of (3.2)-(3.9) as positivity and boundedness for fairly well posed initial conditions. Furthermore, they also justify rigorously that the carbonation front $S(t)$ satisfies a long time behaviour of the type $C_{1} \sqrt{t} \leq S(t) \leq C_{2} \sqrt{t}$, when the exposed boundary conditions are constant, $G(t)=G^{*}, H(t)=H^{*}$, and linked by the condition $G^{*}=\gamma H^{*}$. Numerical simulations of the solution of carbonation problems using the finite element method have been performed in [58, 60].

As the exact solution of the model (3.2)-(3.9) is not available and the best model may be wasted with a bad numerical analysis, in this chapter we provide conditionally stable positive numerical solutions, apart from preserving the qualitative properties of the theoretical solution.

In Section 3.1 of this chapter, after a front-fixing transformation approach, the original problem is transformed into another one where the moving boundary becomes a new unknown of the problem, allowing the possibility to compute the expanding front. We propose a coupled finite difference scheme whose unknowns are both $\mathrm{CO}_{2}$ concentrations, in air and water phases of pores, as well as the square power values of the expanding front. In Section 3.2, stability and positivity of the numerical solution is treated. The monotone increase in time behaviour of the expanding front is shown numerically. We also prove for a fixed time the $\mathrm{CO}_{2}$ concentrations are spatially decreasing from the exposed front to the carbonation front. Section 3.3 deals with a numerical conformation of the $\sqrt{t}$-law assumption. 


\section{2 SPECIES MODEL FOR DAMAGE BY CARBONATION OF REINFORCED CONCRETE STRUCTURES}

Numerical experiments illustrating the shown properties are included in the corresponding sections. Consistency of the proposed numerical scheme with the PDE problem is addressed in Section 3.4.

\subsection{Front-fixing transformation and discretization}

Let us begin this section by transforming the moving boundary problem (3.2)-(3.9) into another one with fixed boundary conditions. The Landau transformation, [26, 49], suggests the substitution

$$
L(t)=S^{2}(t), \quad z(t, x)=\frac{x}{\sqrt{L(t)}}, \quad 0 \leq t \leq T, \quad 0<x<\sqrt{L(t)} .
$$

Using substitution (3.10), the problem (3.2)-(3.9) becomes

$$
\begin{aligned}
& L(t) \frac{\partial W}{\partial t}-L^{\prime}(t) \frac{z}{2} \frac{\partial W}{\partial z}-\kappa_{1} \frac{\partial^{2} W}{\partial z^{2}}=L(t) \beta(\gamma Y-W), \quad 0<t<T, \quad 0<z<1, \\
& L(t) \frac{\partial Y}{\partial t}-L^{\prime}(t) \frac{z}{2} \frac{\partial Y}{\partial z}-\kappa_{2} \frac{\partial^{2} Y}{\partial z^{2}}=-L(t) \beta(\gamma Y-W), \quad 0<t<T, \quad 0<z<1,
\end{aligned}
$$

where

$$
W(t, z)=U(t, x), \quad Y(t, z)=V(t, x) .
$$

In addition, the new boundary conditions take the form

$$
W(t, 0)=G(t), \quad Y(t, 0)=H(t), \quad 0 \leq t \leq T .
$$

The Stefan-like conditions (3.5)-(3.7) are transformed into

$$
\begin{gathered}
L^{\prime}(t)=2 \sqrt{L(t)} \alpha[W(t, 1)]^{p}, \quad 0<t<T, \\
-2 \kappa_{1} \frac{\partial W}{\partial z}(t, 1)=L^{\prime}(t)(1+W(t, 1)), \quad 0<t<T, \\
-2 \kappa_{2} \frac{\partial Y}{\partial z}(t, 1)=L^{\prime}(t) Y(t, 1), \quad 0<t<T,
\end{gathered}
$$

while the initial conditions (3.9) become

$L(0)=L_{0} ; W(0, z)=W_{0}(z)=U_{0}\left(z S_{0}\right) ; Y(0, z)=Y_{0}(z)=V_{0}\left(z S_{0}\right), 0<z<1$. 


\subsection{Front-fixing transformation and discretization}

Note that the transformed problem (3.11)-(3.18) is an initial-fixed boundary problem for a system of two nonlinear parabolic partial differential equations in the bounded fixed domain $(0, T) \times(0,1)$. At this point, we proceed to treat numerically the system (3.11)-3.18) making use of finite difference approximations, which is permissible in accordance with the classical nature of the solution. This result can be derived from a lifting regularity argument presented in [59].

In accordance with the notation presented in Section 1.3, let $N$ and $M$ be positive integers and let us consider the step sizes discretizations $k=\Delta t=T / N$, $h=\Delta z=1 / M$ and the mesh points $\left(t^{n}, z_{j}\right)$, with $t^{n}=n k, z_{j}=j h, 0 \leq n \leq N$, $0 \leq j \leq M$. Numerical approximations of the involved variables are denoted by: $w_{j}^{n} \approx W\left(t^{n}, z_{j}\right), y_{j}^{n} \approx Y\left(t^{n}, z_{j}\right), l^{n} \approx L\left(t^{n}\right)$, while we denote $G^{n}=G\left(t^{n}\right)$, $H^{n}=H\left(t^{n}\right)$.

Assuming that the solution $\{W(t, z), Y(t, z), L(t)\}$ satisfies $W, Y \in C^{1,2}(\Omega)$, where $\Omega=\left\{(t, z) \in \mathbb{R}^{2}: t \in[0, T], z \in[0,1]\right\}$, and $L \in C^{1}[0, T]$, partial derivatives at the interior points are approximated using forward in time and centered in space finite difference expressions:

$$
\begin{gathered}
\frac{w_{j}^{n+1}-w_{j}^{n}}{k} \approx \frac{\partial W}{\partial t}\left(t^{n}, z_{j}\right), \quad \frac{y_{j}^{n+1}-y_{j}^{n}}{k} \approx \frac{\partial Y}{\partial t}\left(t^{n}, z_{j}\right), \quad \frac{l^{n+1}-l^{n}}{k} \approx L^{\prime}\left(t^{n}\right), \\
\frac{w_{j+1}^{n}-w_{j-1}^{n}}{2 h} \approx \frac{\partial W}{\partial z}\left(t^{n}, z_{j}\right), \quad \frac{w_{j-1}^{n}-2 w_{j}^{n}+w_{j+1}^{n}}{h^{2}} \approx \frac{\partial^{2} W}{\partial z^{2}}\left(t^{n}, z_{j}\right) \\
\frac{y_{j+1}^{n}-y_{j-1}^{n}}{2 h} \approx \frac{\partial Y}{\partial z}\left(t^{n}, z_{j}\right), \quad \frac{y_{j-1}^{n}-2 y_{j}^{n}+y_{j+1}^{n}}{h^{2}} \approx \frac{\partial^{2} Y}{\partial z^{2}}\left(t^{n}, z_{j}\right) .
\end{gathered}
$$

To preserve the second order accuracy at the right boundary $z=1$, we take left side approximations with three points:

$$
\frac{3 w_{M}^{n}-4 w_{M-1}^{n}+w_{M-2}^{n}}{2 h} \approx \frac{\partial W}{\partial z}\left(t^{n}, 1\right), \quad \frac{3 y_{M}^{n}-4 y_{M-1}^{n}+y_{M-2}^{n}}{2 h} \approx \frac{\partial Y}{\partial z}\left(t^{n}, 1\right)
$$

Using the approximations (3.19)-(3.21), equations (3.11)-(3.12) become discretized at the interior mesh points in the following way 


\section{2 SPECIES MODEL FOR DAMAGE BY CARBONATION OF REINFORCED CONCRETE STRUCTURES}

$$
\begin{aligned}
& l^{n} \frac{w_{j}^{n+1}-w_{j}^{n}}{k}-\frac{z_{j}}{2} \frac{w_{j+1}^{n}-w_{j-1}^{n}}{2 h}\left(\frac{l^{n+1}-l^{n}}{k}\right)-\kappa_{1} \frac{w_{j-1}^{n}-2 w_{j}^{n}+w_{j+1}^{n}}{h^{2}} \\
& =l^{n} \beta\left(\gamma y_{j}^{n}-w_{j}^{n}\right), \quad 0 \leq n \leq N-1, \quad 1 \leq j \leq M-1, \\
& l^{n} \frac{y_{j}^{n+1}-y_{j}^{n}}{k}-\frac{z_{j}}{2} \frac{y_{j+1}^{n}-y_{j-1}^{n}}{2 h}\left(\frac{l^{n+1}-l^{n}}{k}\right)-\kappa_{2} \frac{y_{j-1}^{n}-2 y_{j}^{n}+y_{j+1}^{n}}{h^{2}} \\
& =-l^{n} \beta\left(\gamma y_{j}^{n}-w_{j}^{n}\right), \quad 0 \leq n \leq N-1, \quad 1 \leq j \leq M-1 .
\end{aligned}
$$

Initial conditions given in (3.18) take the discrete form

$$
l^{0}=S^{2}(0)=S_{0}^{2}, \quad w_{j}^{0}=U_{0}\left(z_{j} S_{0}\right), \quad y_{j}^{0}=V_{0}\left(z_{j} S_{0}\right), \quad 1 \leq j \leq M-1 .
$$

Note that the starting values $w_{M}^{0}$ and $y_{M}^{0}$ are not given and need to be obtained.

Left boundary conditions are discretized as

$$
w_{0}^{n}=G^{n}, \quad y_{0}^{n}=H^{n}, \quad 0 \leq n \leq N
$$

The discretization of the Stefan-like conditions (3.15)-(3.17) takes the form

$$
\begin{gathered}
\frac{l^{n+1}-l^{n}}{k}=2 \alpha\left(l^{n}\right)^{\frac{1}{2}}\left(w_{M}^{n}\right)^{p}, \quad 0 \leq n \leq N-1, \\
-\kappa_{1} \frac{3 w_{M}^{n}-4 w_{M-1}^{n}+w_{M-2}^{n}}{h}=\frac{l^{n+1}-l^{n}}{k}\left(1+w_{M}^{n}\right), \quad 0 \leq n \leq N-1, \\
-\kappa_{2} \frac{3 y_{M}^{n}-4 y_{M-1}^{n}+y_{M-2}^{n}}{h}=\frac{l^{n+1}-l^{n}}{k} y_{M}^{n}, \quad 0 \leq n \leq N-1 .
\end{gathered}
$$

For the sake of clarity, we explain here how to transit from the time level $n$ to $n+1$. Firstly, solving (3.27)-(3.29) for $n=0$ one gets the starting unknown values $w_{M}^{0}$ and $y_{M}^{0}$, as well as $l^{1}$. Then, from the values for the time level $n$, $\left\{w_{j}^{n}, y_{j}^{n}, l^{n} ; 0 \leq j \leq M-1\right\}$, one needs to obtain the remaining values $\left\{w_{M}^{n}, y_{M}^{n}\right\}$ and $\left\{w_{j}^{n+1}, y_{j}^{n+1}, l^{n+1} ; 1 \leq j \leq M-1\right\}$. Note that the nonlinear system 3.27)(3.28) is coupled in the unknowns $w_{M}^{n}$ and $l^{n+1}$. From 3.27) one gets

$$
l^{n+1}=l^{n}+2 k \alpha\left(l^{n}\right)^{\frac{1}{2}}\left(w_{M}^{n}\right)^{p}, \quad 0 \leq n \leq N-1 .
$$

By substituting (3.30) in 3.28) the following nonlinear equation in $w_{M}^{n}$ for each step $n$ must be solved

$$
F_{n}\left(w_{M}^{n}\right)=0, \quad 0 \leq n \leq N
$$


where $F_{n}:[0, \infty[\rightarrow \mathbb{R}$, is given by

$$
F_{n}(\xi)=2 \alpha\left(l^{n}\right)^{\frac{1}{2}} \xi^{p+1}+2 \alpha\left(l^{n}\right)^{\frac{1}{2}} \xi^{p}+\frac{3 \kappa_{1}}{h} \xi-\frac{\kappa_{1}}{h}\left(4 w_{M-1}^{n}-w_{M-2}^{n}\right) .
$$

We solve equation (3.31)-(3.32) using Newton iteration method. Once $w_{M}^{n}$ is calculated solving (3.31)-(3.32), the unknown $l^{n+1}$ is given by (3.30) and $y_{M}^{n}$ is computed using (3.29). In this process, positivity of the involved quantities $w_{M}^{n}, l^{n}$ and $y_{M}^{n}$ has to be proved. This positiveness requirement is fulfilled in Section 3.

Finally, equations (3.23)-(3.24) allow to obtain explicitly the solutions at the interior points at time level $n+1$ as follows

$$
\begin{aligned}
& w_{j}^{n+1}=a_{1, j}^{n} w_{j-1}^{n}+b_{1, j}^{n} w_{j}^{n}+c_{1, j}^{n} w_{j+1}^{n}+k \beta \gamma y_{j}^{n}, \quad 0 \leq n \leq N-1, \quad 1 \leq j \leq M-1 \text {, } \\
& y_{j}^{n+1}=a_{2, j}^{n} y_{j-1}^{n}+b_{2, j}^{n} y_{j}^{n}+c_{2, j}^{n} y_{j+1}^{n}+k \beta w_{j}^{n}, \quad 0 \leq n \leq N-1, \quad 1 \leq j \leq M-1 \text {, }
\end{aligned}
$$

where

$$
\begin{aligned}
& a_{i, j}^{n}=\frac{\kappa_{i} k}{h^{2} l^{n}}-\frac{z_{j}}{4 h} \Delta^{n}, \quad c_{i, j}^{n}=\frac{\kappa_{i} k}{h^{2} l^{n}}+\frac{z_{j}}{4 h} \Delta^{n}, \quad i=1,2, \\
& b_{1, j}^{n}=1-k \beta-\frac{2 \kappa_{1} k}{h^{2} l^{n}}, \quad b_{2, j}^{n}=1-k \beta \gamma-\frac{2 \kappa_{2} k}{h^{2} l^{n}},
\end{aligned}
$$

and

$$
\Delta^{n}=\frac{l^{n+1}}{l^{n}}-1
$$

We summarize the construction of the numerical solution in the procedure exposed in Algorithm 1 .

\subsection{Positivity, stability and monotonicity of the numer- ical solution}

Dealing with concentrations, the positivity of the computed values is not an alternative but a necessity that needs to be guaranteed. We use an inductive method where, under the assumption that values $\left\{w_{j}^{n}, y_{j}^{n}, l^{n} ; 1 \leq j \leq M-1\right\}$ are positive, we show the positivity of the elements of $\left\{w_{j}^{n+1}, y_{j}^{n+1}, l^{n+1} ; 1 \leq j \leq M-1\right\}$, as well as the positivity of the remaining values on the right boundary of the discrete domain, $\left\{w_{M}^{n}, y_{M}^{n}\right\}$. 


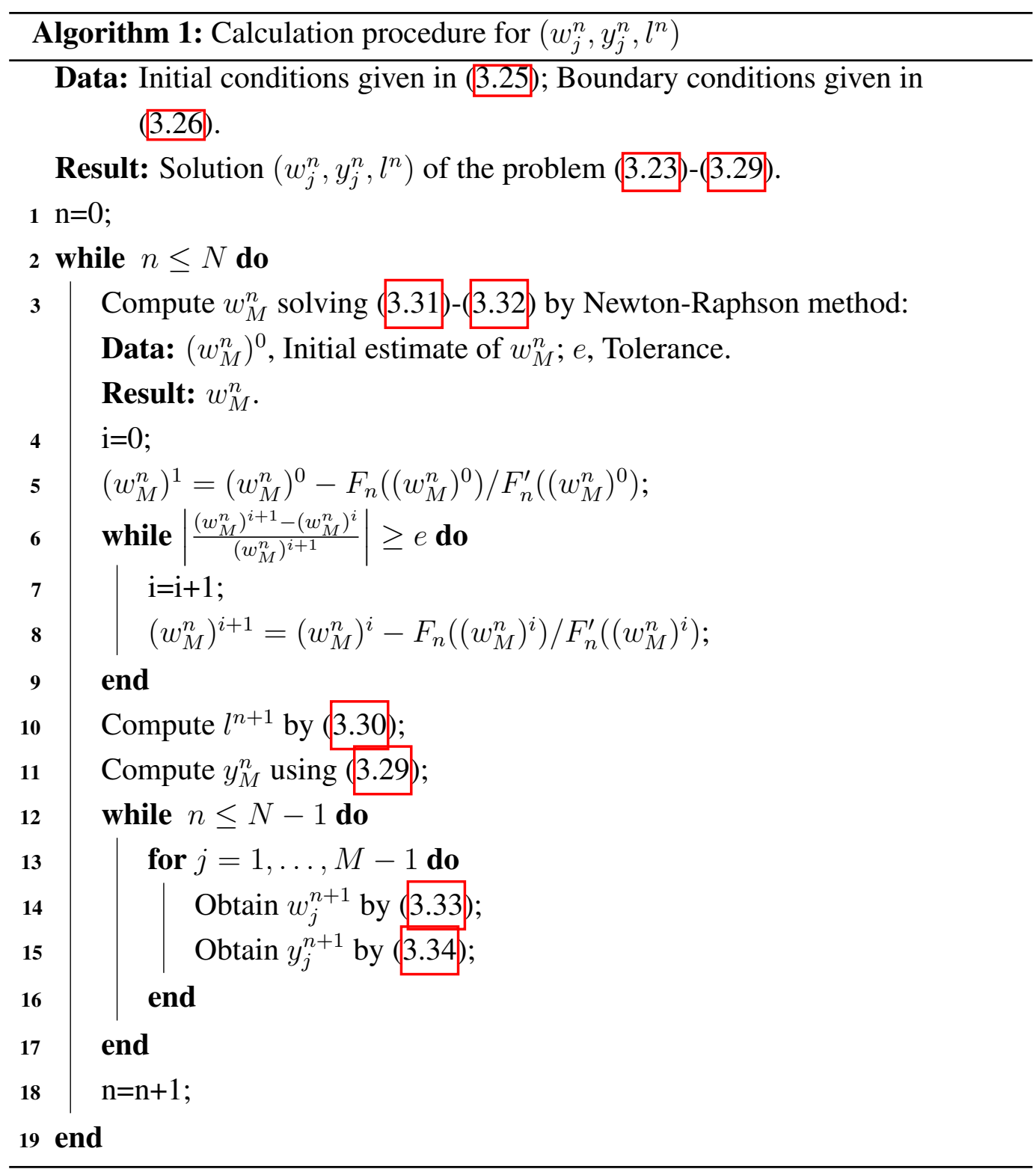


Let us start showing the positivity of the solution $w_{M}^{n}$ of equation 3.31)-(3.32) paying attention to the last term of equation (3.32). Note that from Taylor's theorem one has

$$
w_{M-2}^{n}=w_{M-1}^{n}-h \frac{\partial W}{\partial z}\left(t^{n}, \xi\right) ; \quad(M-2) h<\xi<(M-1) h .
$$

Let $E_{n}$ be defined by

$$
E_{n}=\max \left|\frac{\partial W}{\partial z}\left(t^{n}, z\right)\right|, \quad 0 \leq z \leq 1,
$$

from (3.37) and 3.38

$$
\left|w_{M-2}^{n}-w_{M-1}^{n}\right|<E_{n} h .
$$

Hence, taking $h<3 w_{M-1} / E_{n}$, one gets

$$
4 w_{M-1}^{n}-w_{M-2}^{n}=3 w_{M-1}^{n}+\left(w_{M-1}^{n}-w_{M-2}^{n}\right)>3 w_{M-2}^{n}-E_{n} h>0 .
$$

Let us denote $\delta^{n}=\left(4 w_{M-1}^{n}-w_{M-2}^{n}\right) / 3>0$ and note that $F_{n}(\xi)$ defined by (3.32) is a continuous strictly increasing function satisfying $F_{n}(0)=\left(-3 \kappa_{1} / h\right) \delta^{n}<$ 0 and $F_{n}\left(\delta^{n}\right)>0$, for each $n \geq 0$. Thus, there exists a unique point $\xi_{*}^{n}$ such that $0<\xi_{*}^{n}<\delta^{n}$ and $F_{n}\left(\xi_{*}^{n}\right)=0$. This unique solution of (3.31)- 3.32) is the required value $w_{M}^{n}=\xi_{*}^{n}>0$.

From (3.30) and induction principle, it follows that

$$
0<l^{n}<l^{n+1}, \quad 0 \leq n \leq N-1 \text {. }
$$

From 3.29, one gets

$$
y_{M}^{n}=k\left[\frac{\kappa_{2}\left(4 y_{M-1}^{n}-y_{M-2}^{n}\right)}{3 k \kappa_{2}+h\left(l^{n+1}-l^{n}\right)}\right], 0 \leq n \leq N-1 .
$$

Positivity of $\left(4 y_{M-1}^{n}-y_{M-2}^{n}\right)$ for small enough values of $h$, is obtained in analogous way to the proof of the same result for $\left(4 w_{M-1}^{n}-w_{M-2}^{n}\right)$. Using (3.41) and (3.42) we have that $y_{M}^{n}>0$.

Regarding to the positivity of the remaining $w_{j}^{n+1}$ and $y_{j}^{n+1}, 1 \leq j \leq M-1$, let us study the nonnegativity of the coefficients (3.35) of the scheme (3.33)-(3.34). From 3.35 and 3.41 one gets that $c_{i, j}^{n}>0, i=1,2$, and

$$
b_{1, j}^{n} \geq 1-k \beta-\frac{2 \kappa_{1} k}{h^{2} l^{0}}, \quad b_{2, j}^{n} \geq 1-k \beta \gamma-\frac{2 \kappa_{2} k}{h^{2} l^{0}} .
$$




\section{2 SPECIES MODEL FOR DAMAGE BY CARBONATION OF REINFORCED CONCRETE STRUCTURES}

Then, coefficients $b_{i, j}^{n}, i=1,2$, are positive under the condition

$$
k<k_{0}=\min \left\{k_{1}, k_{2}\right\},
$$

where

$$
k_{1}=\frac{h^{2} l_{0}}{2 \kappa_{1}+h^{2} \beta l_{0}}, \quad k_{2}=\frac{h^{2} l_{0}}{2 \kappa_{2}+h^{2} \beta \gamma l_{0}} .
$$

Now we address the nonnegativity of the coefficients $a_{1, j}^{n}$, whose sign depends on the difference $l^{n+1}-l^{n}$ and, from the discretization of the Stefan-like condition (3.28, this difference involves $w_{M}$ and $\left(4 w_{M-1}-w_{M-2}\right)$.

In order to simplify these relationships, let us consider the right-hand side approximation of the spatial partial derivative of $W(t, z)$ at $\left(t^{n}, 1\right)$

$$
\frac{-3 w_{M}^{n}+4 w_{M+1}^{n}-w_{M+2}^{n}}{2 h}=\frac{\partial W}{\partial z}\left(t^{n}, 1\right)+\mathcal{O}\left(h^{2}\right),
$$

where the artificial values $w_{M+1}^{n}$ and $w_{M+2}^{n}$ vanish because they are outside of the real carbonated region.

In agreement with (3.22), left-hand side backward approximation of the spatial partial derivative of $W(t, z)$ at $\left(t^{n}, 1\right)$ satisfies

$$
\frac{3 w_{M}^{n}-4 w_{M-1}^{n}+w_{M-2}^{n}}{2 h}=\frac{\partial W}{\partial z}\left(t^{n}, 1\right)+\mathcal{O}\left(h^{2}\right) .
$$

From (3.46)-(3.47), one gets

$$
6 w_{M}^{n}=4 w_{M-1}^{n}-w_{M-2}^{n}+\mathcal{O}\left(h^{3}\right) .
$$

Using (3.28) and (3.48), it follows that

$$
l^{n+1}-l^{n}=\frac{k \kappa_{1}}{h} \frac{3 w_{M}^{n}}{1+w_{M}^{n}}+\mathcal{O}\left(k h^{2}\right),
$$

and from 3.35) and (3.49), using that $0<z_{j} \leq 1$,

$$
a_{1, j}^{n} \geq \frac{\kappa_{1} k}{h^{2} l^{n}}\left(\frac{4+w_{M}^{n}}{4\left(1+w_{M}^{n}\right)}+\mathcal{O}\left(h^{3}\right)\right) .
$$

Hence, for small enough values of $k$ and $h$, coefficients $a_{1, j}^{n}$ are positive. Finally, from the expression 3.35 for $a_{2, j}^{n}$, managing the values of $y_{M}^{n}$ and $\left(4 y_{M-1}^{n}-y_{M-2}^{n}\right)$ in analogous way to the previous study with $w_{j}^{n}$, one gets

$$
6 y_{M}^{n}=4 y_{M-1}^{n}-y_{M-2}^{n}+\mathcal{O}\left(h^{3}\right),
$$




$$
l^{n+1}-l^{n}=\frac{3 k \kappa_{2}}{h}+\mathcal{O}\left(k h^{2}\right),
$$

and

$$
a_{2, j}^{n} \geq \frac{\kappa_{2} k}{h^{2} l^{n}}\left(\frac{1}{4}+\mathcal{O}\left(h^{3}\right)\right)
$$

Summarizing, the following result has been established:

Theorem 4. With previous notation, for small enough values of the step sizes $h$ and $k$ linked by the condition (3.44)-(3.45), the following conclusions hold true:

i) Concentration solutions of the scheme (3.33)-(3.34) $w_{j}^{n}$ and $y_{j}^{n}$ are positive for $1 \leq j \leq M-1,1 \leq n \leq N$.

ii) Concentrations at the right boundary $w_{M}^{n}$ and $y_{M}^{n}$ are positive.

iii) The moving carbonation front is positive and increasing, $0<l^{0}<l^{1}$ $<\ldots<l^{N}$.

The positivity guaranteed by Theorem 4 is not unconditional and the following example shows that this condition can not be removed.

Example 1. In accordance with [60], consider the carbonation model (3.2)(3.9) with parameters listed in the Table 3.1 and time horizon $T=15$ years. For $h=0.05$, one gets $k_{1}=0.0062$ and $k_{2}=0.0625$. Taking $k=0.0075$, the positivity condition is broken. Figure 3.1 shows that positivity does not hold. Units in x-axe are taken in $\mathrm{cm}$ and $\mathrm{y}$-axe in $10^{-6} \mathrm{~g} \mathrm{~cm}^{-3}$.

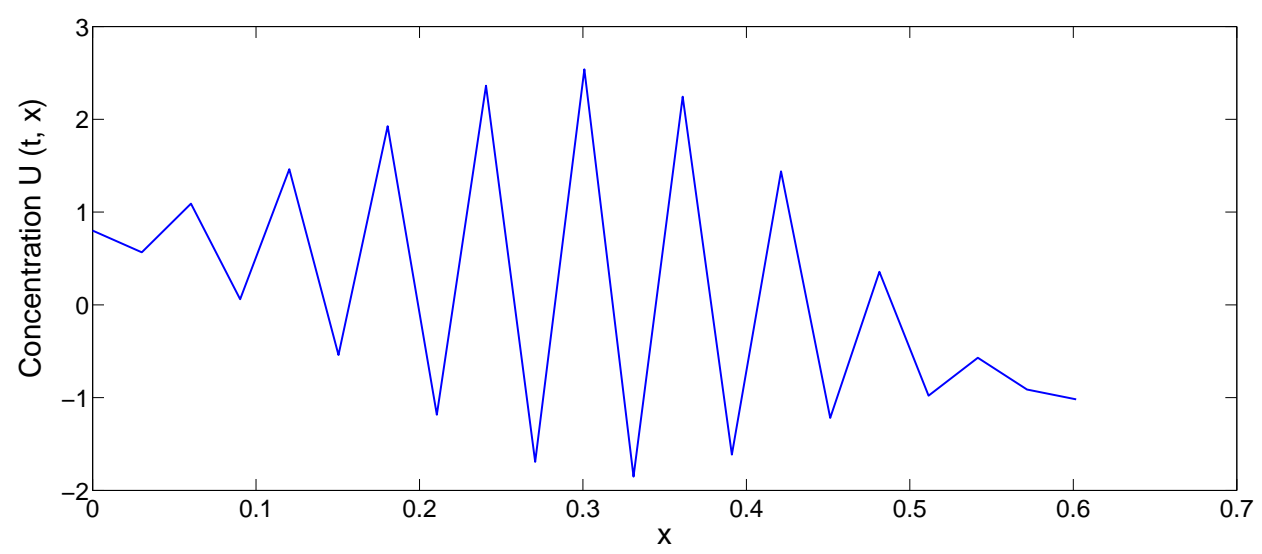

Figure 3.1: Numerical solution of Example 1 for $t=0.375$ years, when positivity condition is broken. 


\section{2 SPECIES MODEL FOR DAMAGE BY CARBONATION OF REINFORCED CONCRETE STRUCTURES}

\begin{tabular}{ll}
\hline \hline Magnitude & Value \\
Initial concentrations $\left(10^{-6} \times \mathrm{g} \mathrm{cm}^{-3}\right)$ & \\
$U_{0}(x), 0<x<S_{0}$ & $0.80-1.50 x$ \\
$V_{0}(x), 0<x<S_{0}$ & $200000-398000 x$ \\
\hline Exposed boundary concentrations $\left(10^{-6} \times \mathrm{g} \mathrm{cm}^{-3}\right)$ & \\
$G(t)$ & 0.80 \\
$H(t)$ & 200000 \\
\hline Diffusion constants $\left(10^{8} \times \mathrm{cm}^{2}\right.$ year $\left.^{-1}\right)$ & \\
$\kappa_{1}$ & 0.05 \\
$\kappa_{2}$ & 0.005 \\
\hline Model parameters & \\
$\alpha$ & 1.00 \\
$\beta \quad$ year & \\
$\gamma$ & 0.001 \\
$p$ & $5 \times 10^{-6}$ \\
\hline Initial position of carbonation front $(\mathrm{cm})$ & 2.00 \\
$S_{0}$ & \\
\hline \hline
\end{tabular}

Table 3.1: Data for numerical examples.

Apart from the positivity of the numerical solution, a crucial requirement is the stability of such numerical solution. For the sake of clarity in the presentation and because of the existence in the literature of several definitions of stability, the notion (1.18) presented in Chapter 1 is applied here. As the stability is related to the boundedness of the numerical solution, we will use the so-called supremum norm of a vector $x=\left(x_{1}, x_{2}, \ldots, x_{n}\right)^{T}$ in $\mathbb{R}^{n}$, defined as $\|x\|_{\infty}=\max \left(\left|x_{1}\right|,\left|x_{2}\right|, \ldots,\left|x_{n}\right|\right)$.

Definition 2. With previous notation, let us denote the vectors of $\mathrm{CO}_{2}$ concentrations $w^{n}=\left[w_{0}^{n}, w_{1}^{n}, \ldots, w_{M}^{n}\right]^{T}$ and $y^{n}=\left[y_{0}^{n}, y_{1}^{n}, \ldots, y_{M}^{n}\right]^{T}$. In accordance with (1.18), we say that the numerical solution $\left\{w^{n}, y^{n}, \quad 0 \leq n \leq N\right\}$ is $\|\cdot\|_{\infty}$-stable if there exist positive constants $C_{1}$ and $C_{2}$ independent of $n, k$ and $h$, such that

$$
\left\|w^{n}\right\|_{\infty} \leq C_{1}, \quad\left\|y^{n}\right\|_{\infty} \leq C_{2}, \quad 0 \leq n \leq N
$$

Now we show that under the positivity conditions of Theorem 4 the numerical 
solution $\left\{w_{j}^{n}, y_{j}^{n}\right\}$ is $\|\cdot\|_{\infty}$-stable. Let $G(t)$ and $H(t)$ be the left boundary conditions given by (3.4) and let $U_{0}(x)$ and $V_{0}(x)$ be the initial conditions given by (3.9). Let $\tilde{G}$ and $\tilde{H}$ be positive upper bounds such that

$$
\left.\begin{array}{l}
G(t) \leq \tilde{G}, \quad H(t) \leq \tilde{H}, \quad 0 \leq t \leq T, \\
U_{0}\left(z_{j} S_{0}\right)=w_{j}^{0} \leq \tilde{G}, \quad V_{0}\left(z_{j} S_{0}\right)=y_{j}^{0} \leq \tilde{H}, \quad 1 \leq j \leq M-1, \\
\tilde{G}=\gamma \tilde{H} .
\end{array}\right\}
$$

From (3.48) and (3.51) evaluated at $n=0$ it follows

$$
w_{M}^{0}<\frac{2}{3} w_{M-1}^{0} \leq \tilde{G}, \quad y_{M}^{0}<\frac{2}{3} y_{M-1}^{0} \leq \tilde{H} .
$$

Once we have the bound at the time level $n=0$, we prove using the induction principle the boundedness of the numerical solution for all the time levels. Let us assume the induction hypothesis

$$
w_{j}^{n} \leq \tilde{G}, \quad y_{j}^{n} \leq \tilde{H}, \quad 1 \leq j \leq M, \quad \tilde{G}=\gamma \tilde{H} .
$$

From (3.33)-(3.34) and (3.35) under the conditions of Theorem 4 one gets, for $1 \leq j \leq M-1$,

$$
\begin{aligned}
& w_{j}^{n+1} \leq\left(a_{1, j}^{n}+b_{1, j}^{n}+c_{1, j}^{n}\right) \tilde{G}+k \beta \gamma \tilde{H}=(1-k \beta) \tilde{G}+k \beta \gamma \tilde{G}=\tilde{G}, \\
& y_{j}^{n+1} \leq\left(a_{2, j}^{n}+b_{2, j}^{n}+c_{2, j}^{n}\right) \tilde{H}+k \beta \tilde{G}=(1-k \beta \gamma) \tilde{H}+k \beta \tilde{G}=\tilde{H} .
\end{aligned}
$$

Furthermore, using (3.48) and (3.51), together with (3.58)-(3.59) for $j=M-1$, it follows that $w_{M}^{n+1} \leq \tilde{G}$ and $y_{M}^{n+1} \leq \tilde{H}$.

Summarizing the following conditional stability result has been established:

Theorem 5. With previous notation, for small enough values of $h$ and $k$ satisfying the positivity step size condition (3.44)-(3.45), the numerical solution of scheme (3.33)-(3.34) is $\|\cdot\|_{\infty}$-stable.

As it has been shown in Example 1, where the positivity does not occur when the positivity condition (3.44)-(3.45) was broken, in the following example we show that when the positivity condition is satisfied, then we have both positivity and $\|\cdot\|_{\infty}$-stability.

Example 2. With the notation and the parameters of Example 1, and step sizes $h=0.05$ and $k=0.005$, the stability of the solutions $U(t, x)$ and $V(t, x)$ is 


\section{2 SPECIES MODEL FOR DAMAGE BY CARBONATION OF REINFORCED CONCRETE STRUCTURES}

guaranteed as it is shown in Figure 3.2. Units in $\mathrm{x}$-axe are taken in $\mathrm{cm}$ and $\mathrm{y}$-axe in $10^{-6} \mathrm{~g} \mathrm{~cm}^{-3}$.
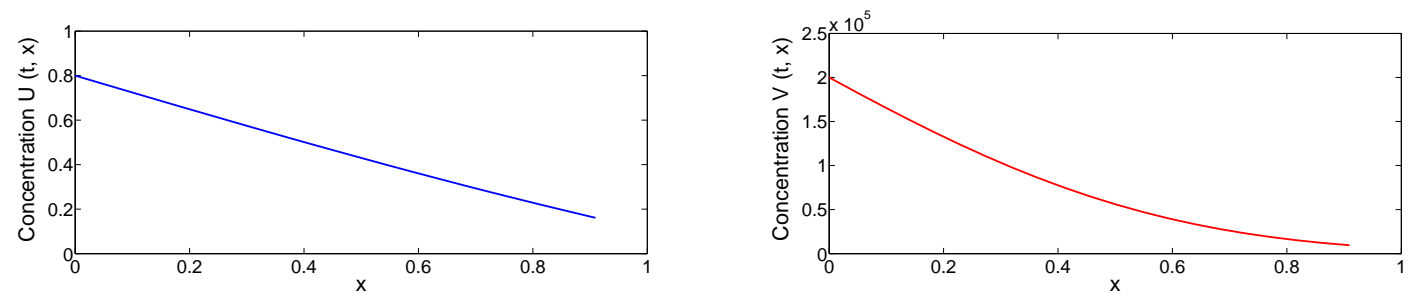

Figure 3.2: Numerical solution of $U(t, x)$ and $V(t, x)$ in Example 2 for $t=13$ years, under stability condition.

An important property of the numerical solution is its monotone decreasing behaviour with respect to the space from the exposed boundary to the carbonation front at each time level under the positivity step size conditions. For the sake of clarity in the presentation and the concept of monotone schemes has been used earlier in the literature [35], we introduce the following definition:

Definition 3. With previous notation, we say that the numerical scheme (3.33)(3.34) is spatial monotone preserving, if assuming that the numerical solution is spatial monotone decreasing at time level $n, 0 \leq n \leq N-1$, i. e.,:

$$
w_{j+1}^{n} \leq w_{j}^{n}, \quad y_{j+1}^{n} \leq y_{j}^{n}, \quad 0 \leq j \leq M-1,
$$

then, one satisfies

$$
w_{j+1}^{n+1} \leq w_{j}^{n+1}, \quad y_{j+1}^{n+1} \leq y_{j}^{n+1}, \quad 0 \leq j \leq M-1 .
$$

Now we state that, under the positivity constraints (3.44)-(3.45) on the coefficients (3.35) together with an additional condition for concentrations at the exposed boundary, the numerical scheme is spatial monotone preserving. This condition imposes that $G(t)$ and $H(t)$ are time monotone non decreasing functions satisfying

$$
G(t)=\gamma H(t), \quad 0 \leq t \leq T \text {. }
$$

From equation (3.33), the positivity of coefficients $a_{1, j}^{n}, b_{1, j}^{n}, c_{1, j}^{n}$ and the induction hypothesis of Definition 3 , it follows that $w_{j-1}^{n} \geq w_{j}^{n}, w_{j}^{n} \geq w_{j+1}^{n}$, 


$$
\begin{aligned}
& w_{j}^{n+1} \geq a_{1, j}^{n} w_{j}^{n}+b_{1, j}^{n} w_{j}^{n}+c_{1, j}^{n} w_{j}^{n}+k \beta \gamma y_{j}^{n} \\
& =\left(1-k \beta-\frac{\kappa_{1} k}{h^{2} l^{n}}-\frac{j}{4} \Delta^{n}\right) w_{j}^{n}+c_{1, j}^{n} w_{j}^{n}+k \beta \gamma y_{j}^{n}, \quad 1 \leq j \leq M-1,
\end{aligned}
$$

and

$$
\begin{aligned}
& w_{j+1}^{n+1} \leq a_{1, j+1}^{n} w_{j+1}^{n}+b_{1, j}^{n} w_{j+1}^{n}+c_{1, j+1}^{n} w_{j+1}^{n}+k \beta \gamma y_{j+1}^{n} \\
= & \left(1-k \beta-\frac{\kappa_{1} k}{h^{2} l^{n}}+\frac{j+1}{4} \Delta^{n}\right) w_{j+1}^{n}+a_{1, j+1}^{n} w_{j+1}^{n}+k \beta \gamma y_{j+1}^{n}, \quad 1 \leq j \leq M-2 .
\end{aligned}
$$

From (3.63) and (3.64) and the hypothesis condition of Definition 3 it follows that

$$
w_{j+1}^{n+1}-w_{j}^{n+1} \leq\left(b_{1, j}^{n}+\frac{1}{4} \Delta^{n}\right)\left(w_{j+1}^{n}-w_{j}^{n}\right)+k \beta \gamma\left(y_{j+1}^{n}-y_{j}^{n}\right) \leq 0, \quad 1 \leq j \leq M-2 .
$$

Furthermore $w_{M}^{n+1}<w_{M-1}^{n+1}$ due to 3.56. Using equation 3.33 for $j=1$, the hypothesis of Definition 3 and assuming that $G(t)$ and $H(t)$ are monotone non decreasing functions satisfying (3.62), it follows

$$
\begin{gathered}
w_{1}^{n+1} \leq\left(a_{1,1}^{n}+b_{1,1}^{n}+c_{1,1}^{n}\right) w_{0}^{n}+k \beta \gamma y_{0}^{n} \\
\leq(1-k \beta) G^{n}+k \beta \gamma H^{n}=G^{n} \leq G^{n+1}=w_{0}^{n+1} .
\end{gathered}
$$

The monotonicity of $\left\{y_{j}^{n+1}\right\}$ can be shown in an analogous way as for $\left\{w_{j}^{n+1}\right\}$. The following result has been established.

Theorem 6. With previous notation, under the positivity conditions (3.44-(3.45), assuming that the concentrations at the exposed boundary are monotone non decreasing functions such that $G(t)=\gamma H(t)$, then the numerical scheme (3.33)(3.34) is spatial monotone preserving.

Next Example 3 illustrates the decreasing monotonicity behaviour of both concentrations $U(t, x)$ and $V(t, x)$ in the space variable when the time is fixed.

Example 3. With data of Table 3.1 and taking step sizes $h=0.05$ and $k=$ 0.005 satisfying the monotonicity requirements of Theorem 6 Figures 3.3 and 3.4 


\section{2 SPECIES MODEL FOR DAMAGE BY CARBONATION OF REINFORCED CONCRETE STRUCTURES}

show the monotone behaviour of the functions $U\left(t_{i}, x\right)$ and $V\left(t_{i}, x\right)$ corresponding to the mass concentrations of $\mathrm{CO}_{2}$ in air and water, respectively. Here $t_{i}, 1 \leq i \leq$ 5 , represent four equidistant fixed values of time. Note also that the carbonation zone is increasing with time, according to the spreading of the propagation front $S(t)$, see Theorem 4 - iii).

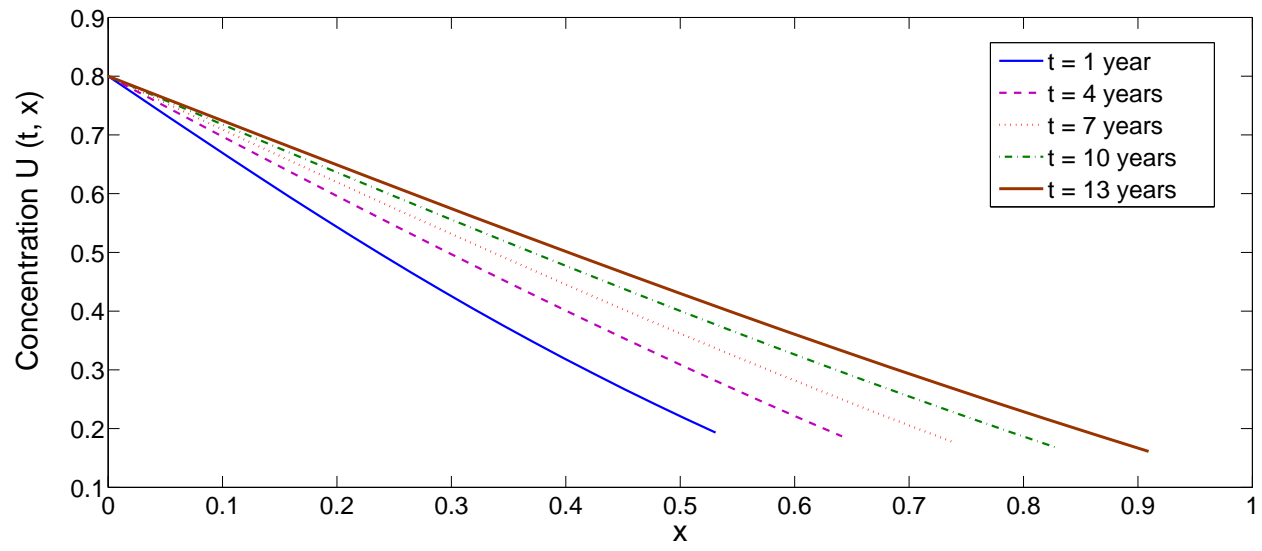

Figure 3.3: Numerical solution $U(t, x)$ of Example 3 for several equidistant times $t_{i}$.

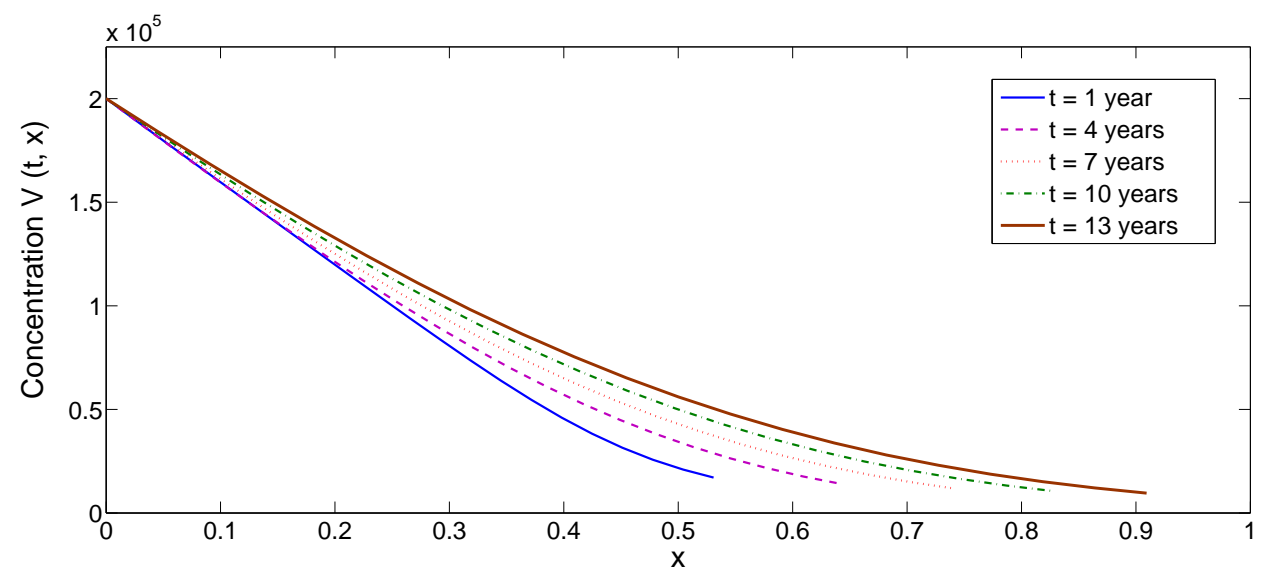

Figure 3.4: Numerical solution $V(t, x)$ of Example 3 for several equidistant times $t_{i}$.

\subsection{Numerical evidence of the $\sqrt{t}$-law of propagation}

In this Section we confirm numerically, under appropriated positivity conditions, that the proposed numerical solution behaves as the theoretical solution suggested. 
In fact, in the next example, according to data from [60], we match the numerical solution of the carbonation front as a function of the type $C \sqrt{t}$.

Example 4. With the same parameters and step size values of Example 2, Table 3.2 shows long time values of the carbonation front $S(t)$ on of time, with a time horizon of $T=35$ years. These points $\left(t_{i}, S\left(t_{i}\right)\right)$ have been fitted to a curve with two parameters of the type $S(t)=a t^{b}$. The best fit (in the least square sense) is matched by $a=0.2715$ and $b=0.4568$, and the coefficient of determination $R^{2}=0.9999$. This numerical experiment illustrates the agreement with the behaviour of the theoretical solution.

\begin{tabular}{llllll}
\hline \hline$t_{i}$ (years) & 33.00 & 33.50 & 34.00 & 34.50 & 35.00 \\
\hline$S\left(t_{i}\right)(\mathbf{c m})$ & 1.3407 & 1.3499 & 1.3591 & 1.3682 & 1.3772 \\
\hline \hline
\end{tabular}

Table 3.2: Carbonation depth for several times.

\subsection{Consistency}

As it has been recalled in Sections 1.3 and 2.2, consistency of a numerical scheme with a PDE problem signifies that the theoretical solution approximates well the numerical scheme as the grid spacings go to zero. Accordingly, a numerical scheme can be consistent with an equation and not with another one, see [72], Chapter 2, and [76], Chapter 1.

Problem (3.11)-(3.17) can be written in vector form as $\mathcal{L}(W, Y, L)$ $=\left(\mathcal{L}_{1}(W, Y, L), \mathcal{L}_{2}(W, Y, L), \mathcal{L}_{3}(W, L), \mathcal{L}_{4}(W, L), \mathcal{L}_{5}(Y, L)\right)=0$, where

$$
\begin{aligned}
& \mathcal{L}_{1}(W, Y, L)=L(t) \frac{\partial W}{\partial t}-L^{\prime}(t) \frac{z}{2} \frac{\partial W}{\partial z}-\kappa_{1} \frac{\partial^{2} W}{\partial z^{2}} \\
& -L(t) \beta(\gamma Y-W)=0, \quad t>0,0<z<1, \\
& \mathcal{L}_{2}(W, Y, L)=L(t) \frac{\partial Y}{\partial t}-L^{\prime}(t) \frac{z}{2} \frac{\partial Y}{\partial z}-\kappa_{2} \frac{\partial^{2} Y}{\partial z^{2}} \\
& +L(t) \beta(\gamma Y-W)=0, \quad t>0,0<z<1, \\
& \mathcal{L}_{3}(W, L)=L^{\prime}(t)-2(L(t))^{\frac{1}{2}} \alpha[W(t, 1)]^{p}=0, \quad t>0,
\end{aligned}
$$




$$
\begin{gathered}
\mathcal{L}_{4}(W, L)=L^{\prime}(t)(1+W(t, 1))+2 \kappa_{1} \frac{\partial W}{\partial z}(t, 1)=0, \quad t>0 \\
\mathcal{L}_{5}(Y, L)=L^{\prime}(t) Y(t, 1)+2 \kappa_{2} \frac{\partial Y}{\partial z}(t, 1)=0, \quad t>0
\end{gathered}
$$

and the finite difference scheme (3.23)-3.29), also can be written in a compact way as $\ell(w, y, l)=\left(\ell_{1}(w, y, l), \ell_{2}(w, y, l), \ell_{3}(w, l), \ell_{4}(w, l), \ell_{5}(y, l)\right)$, where:

$$
\begin{aligned}
& \ell_{1}(w, y, l)=l^{n} \frac{w_{j}^{n+1}-w_{j}^{n}}{k}-\frac{z_{j}}{2} \frac{w_{j+1}^{n}-w_{j-1}^{n}}{2 h}\left(\frac{l^{n+1}-l^{n}}{k}\right)- \\
& \kappa_{1} \frac{w_{j-1}^{n}-2 w_{j}^{n}+w_{j+1}^{n}}{h^{2}}-l^{n} \beta\left(\gamma y_{j}^{n}-w_{j}^{n}\right)=0, \quad 0 \leq n \leq N-1, \quad 1 \leq j \leq M-1,
\end{aligned}
$$

$$
\begin{aligned}
& \ell_{2}(w, y, l)=l^{n} \frac{y_{j}^{n+1}-y_{j}^{n}}{k}-\frac{z_{j}}{2} \frac{y_{j+1}^{n}-y_{j-1}^{n}}{2 h}\left(\frac{l^{n+1}-l^{n}}{k}\right)- \\
& \kappa_{2} \frac{y_{j-1}^{n}-2 y_{j}^{n}+y_{j+1}^{n}}{h^{2}}+l^{n} \beta\left(\gamma y_{j}^{n}-w_{j}^{n}\right)=0, \quad 0 \leq n \leq N-1,1 \leq j \leq M-1,
\end{aligned}
$$

$$
\begin{gathered}
\ell_{3}(w, l)=\frac{l^{n+1}-l^{n}}{k}-2 \alpha\left(l^{n}\right)^{\frac{1}{2}}\left(w_{M}^{n}\right)^{p}=0, \quad 0 \leq n \leq N-1 \\
\ell_{4}(w, l)=\kappa_{1} \frac{3 w_{M}^{n}-4 w_{M-1}^{n}+w_{M-2}^{n}}{h}+\frac{l^{n+1}-l^{n}}{k}\left(1+w_{M}^{n}\right)=0, \quad 0 \leq n \leq N-1, \\
\ell_{5}(y, l)=\kappa_{2} \frac{3 y_{M}^{n}-4 y_{M-1}^{n}+y_{M-2}^{n}}{h}+\frac{l^{n+1}-l^{n}}{k} y_{M}^{n}=0, \quad 0 \leq n \leq N-1
\end{gathered}
$$

In accordance with [72], scheme $\ell(w, y, l)$ is said to be consistent with problem $\mathcal{L}(W, Y, L)$ if the local truncation error $T_{j}^{n}(W, Y, L)=\left(T(1)_{j}^{n}, T(2)_{j}^{n}, T(3)_{j}^{n}, T(4)_{j}^{n}\right.$, $\left.T(5)_{j}^{n}\right)$,

$$
\begin{gathered}
T(1)_{j}^{n}(W, Y, L)=\ell_{1}\left(W_{j}^{n}, Y_{j}^{n}, L^{n}\right)-\mathcal{L}_{1}\left(W_{j}^{n}, Y_{j}^{n}, L^{n}\right), \\
T(2)_{j}^{n}(W, Y, L)=\ell_{2}\left(W_{n}^{j}, Y_{j}^{n}, L^{n}\right)-\mathcal{L}_{2}\left(W_{j}^{n}, Y_{j}^{n}, L^{n}\right), \\
T(3)_{j}^{n}(W, L)=\ell_{3}\left(W_{j}^{n}, L^{n}\right)-\mathcal{L}_{3}\left(W_{j}^{n}, L^{n}\right), \\
T(4)_{j}^{n}(W, L)=\ell_{4}\left(W_{j}^{n}, L^{n}\right)-\mathcal{L}_{4}\left(W_{j}^{n}, L^{n}\right), \\
T(5)_{j}^{n}(Y, L)=\ell_{5}\left(Y_{j}^{n}, L^{n}\right)-\mathcal{L}_{5}\left(Y_{j}^{n}, L^{n}\right),
\end{gathered}
$$


tends to zero as $k \rightarrow 0, h \rightarrow 0$, where $W_{j}^{n}=W\left(t^{n}, z_{j}\right), Y_{j}^{n}=Y\left(t^{n}, z_{j}\right)$ and $L^{n}=L\left(t^{n}\right)$ are the values of the exact solution of problem 3.11$)-(3.17)$ of both the PDE and the free boundary respectively at the point $\left(t^{n}, z_{j}\right)$. We assume that the exact solution $\{W(t, z), Y(t, z), L(t)\}$ satisfies the property $W, Y \in C^{2,4}(\Omega)$, and $L \in C^{2}[0, T]$.

Let us first consider the components $T(1)_{j}^{n}$ and $T(2)_{j}^{n}$ of the local truncation error. By using Taylor's expansion about $\left(t^{n}, z_{j}\right)$ one gets:

$$
\begin{aligned}
& T(1)_{j}^{n}(W, Y, L)=L^{n} E_{j}^{n}(2) k-\frac{z_{j}}{2} \frac{\partial W}{\partial z}\left(t^{n}, z_{j}\right) k E^{n}(1) \\
& -\frac{z_{j}}{2} L^{\prime}\left(t^{n}\right) E_{j}^{n}(3)-\frac{z_{j}}{2} E^{n}(1) E_{j}^{n}(3) k h^{2}-\kappa_{1} E_{j}^{n}(4) h^{2}, \\
& T(2)_{j}^{n}(W, Y, L)=L^{n} E_{j}^{n}(5) k-\frac{z_{j}}{2} \frac{\partial Y}{\partial z}\left(t^{n}, z_{j}\right) k E^{n}(1) \\
& -\frac{z_{j}}{2} L^{\prime}\left(t^{n}\right) E_{j}^{n}(6)-\frac{z_{j}}{2} E^{n}(1) E_{j}^{n}(6) k h^{2}-\kappa_{2} E_{j}^{n}(7) h^{2},
\end{aligned}
$$

where:

$$
\begin{gathered}
E^{n}(1)=\frac{1}{2} \frac{d^{2} L}{d t^{2}}\left(\tau^{1}\right), \quad E_{j}^{n}(2)=\frac{1}{2} \frac{\partial^{2} W}{\partial t^{2}}\left(\tau^{2}, z_{j}\right), \quad E_{j}^{n}(5)=\frac{1}{2} \frac{\partial^{2} Y}{\partial t^{2}}\left(\tau^{3}, z_{j}\right), \\
t^{n}<\tau^{i}<t^{n+1}, \quad i=1,2,3, \\
E_{j}^{n}(3)=\frac{1}{6} \frac{\partial^{3} W}{\partial z^{3}}\left(t^{n}, \xi_{1}\right), \quad E_{j}^{n}(4)=\frac{1}{12} \frac{\partial^{4} W}{\partial z^{4}}\left(t^{n}, \xi_{2}\right), \quad E_{j}^{n}(6)=\frac{1}{6} \frac{\partial^{3} Y}{\partial z^{3}}\left(t^{n}, \xi_{3}\right), \\
E_{j}^{n}(7)=\frac{1}{12} \frac{\partial^{4} Y}{\partial z^{4}}\left(t^{n}, \xi_{4}\right), \quad z_{j-1}<\xi_{i}<z_{j+1}, \quad i=1,2,3,4, \\
E_{j}^{n}(8)=\frac{1}{3} \frac{\partial^{3} W}{\partial z^{3}}\left(t^{n}, \xi_{5}\right), \quad E_{j}^{n}(9)=\frac{1}{3} \frac{\partial^{3} Y}{\partial z^{3}}\left(t^{n}, \xi_{6}\right), \quad z_{M-2}<\xi_{i}<z_{M}, \quad i=5,6.8
\end{gathered}
$$

Hence, $T(1)_{j}^{n}(W, Y, L)$ and $T(2)_{j}^{n}(W, Y, L)$ satisfy

$$
\begin{aligned}
& T(1)_{j}^{n}(W, Y, L)=\mathcal{O}(k)+\mathcal{O}\left(h^{2}\right), \\
& T(2)_{j}^{n}(W, Y, L)=\mathcal{O}(k)+\mathcal{O}\left(h^{2}\right) .
\end{aligned}
$$

The remaining components of the truncation error are

$$
T(3)_{j}^{n}(W, L)=k E^{n}(1),
$$




\section{2 SPECIES MODEL FOR DAMAGE BY CARBONATION OF REINFORCED CONCRETE STRUCTURES}

$$
\begin{gathered}
T(4)_{j}^{n}(W, L)=k E^{n}(1)\left(1+W\left(t^{n}, z_{M}\right)\right)-2 h^{2} \kappa_{1} E_{j}^{n}(8), \\
T(5)_{j}^{n}(Y, L)=k E^{n}(1) Y\left(t^{n}, z_{M}\right)-2 h^{2} \kappa_{2} E_{j}^{n}(9) .
\end{gathered}
$$

Thus, it holds

$$
\begin{gathered}
T(3)_{j}^{n}(W, L)=\mathcal{O}(k), \\
T(4)_{j}^{n}(W, L)=\mathcal{O}(k)+\mathcal{O}\left(h^{2}\right), \\
T(5)_{j}^{n}(Y, L)=\mathcal{O}(k)+\mathcal{O}\left(h^{2}\right) .
\end{gathered}
$$

Summarizing, the following result has been established:

Theorem 7. With previous notation, the scheme $\ell(w, y, l)$ is consistent with the problem $\mathcal{L}(W, Y, L)$ and the local truncation error behaves as:

$$
T_{j}^{n}(W, Y, L)=\mathcal{O}(k)+\mathcal{O}\left(h^{2}\right) .
$$

Remark 1. Note that the coefficients of (3.35), carbonation front $l^{n+1}$ and the $\mathrm{CO}_{2}$ concentration in water $y_{M}^{n}$ at the propagation front, are distorted by the computation of the $\mathrm{CO}_{2}$ concentration in air $w_{M}^{n}$ at the propagation front, using NewtonRaphson method to solve equation (3.31)-(3.32). This means that above we studied the consistency of the scheme resulting of replacing in (3.23)-(3.29) the theoretical value $w_{M}^{n}$ by the numerical one $\tilde{w}_{M}^{n}$ obtained by the Newton-Raphson method. Standard analysis based on the mean value theorem shows that the error of order $p$ motivated by equation (3.30) is linearly transmitted to the other variables adding a local error of order $\mathcal{O}\left(\left|w_{M}^{n}-\tilde{w}_{M}^{n}\right|^{p}\right)$ to the expression (3.95).

Remark 2. The real initial deterioration state of the concrete in the carbonation process is measured in terms of the value $L(0)=S^{2}(0)=S_{0}^{2}$. This value has influence in the step size conditions (3.44)-(3.45) in order to guarantee positivity, stability and monotonicity, that cannot be removed as Example 1 shows. However, this fact does not mean a limitation in our study and its applications, when $S(0)$ is small enough, because starting from any initial position of the carbonation front, using the substitution

$$
\tau=\frac{\kappa_{1} t}{L(0)}, \quad \lambda(\tau)=\frac{L(t)}{L(0)}
$$

the problem becomes independent of this situation, as well as of the quality of diffusion given by the coefficient $\kappa_{1}$. 


\section{6 species model for damage by carbonation of reinforced concrete structures}

As explained in the previous chapter, the effect of concrete carbonation in buildings and civil works is a relevant issue in civil engineering construction and architecture due to the deterioration of building materials and its potential incidence in the citizens safety. This matter is also related to environment, public health and ecology because the carbonation effect is in part due to the influence of traffic and industries. This problem may be regarded as a particular case inside of the wide area of materials corrosion. Apart from the concrete carbonation, other damaging processes are those related to the effects of sulfuric acid attack, [33], or chloride penetration in concrete structures, [64, 85].

The annual carbon dioxide concentration growth rate has increased from 1.4 parts per million (ppm) per year during the period 1960-2005 to 1.9 ppm per year during the period 1995-2005. Following the current tendency, studies estimate that atmospheric $\mathrm{CO}_{2}$ concentration could increase from 379 ppm in 2005 over 1000 ppm by the year 2100, resulting in an increase of corrosion risk, see [8, 73]. 


\section{6 SPECIES MODEL FOR DAMAGE BY CARBONATION OF REINFORCED CONCRETE STRUCTURES}

The presence of carbon dioxide under normal atmospheric conditions and also emitted as industrial output, enters the gaseous phase of the pores. Carbon dioxide is transported by diffusion and dissolved in the aqueous phase, where it is further transported towards the place where carbonation reaction takes place. The other reactant, calcium hydroxide, is initially in the solid matrix of cement. By means of a dissolution process, it arrives in the aqueous phase of the pores and reacts with $\mathrm{CO}_{2}$ present in the aquaeus phase. The reaction products are water together with calcium carbonate. Several experimental studies have addressed the problem of concrete carbonation, studying the behaviour of the position of the interface that separates the carbonated and non carbonated part, for large times, [14, 41, 42, 61, 79. 80].

The concrete carbonation depth, as discussed in Chapter 3, needs to be estimated and there is need of models capable to predict the depth of $\mathrm{CO}_{2}$ penetration in concrete structures accurately, see [58]. In [58, 59, 60], the authors propose a moving interface model to forecast the position of the carbonation front and the profiles of the active concentrations, showing qualitative properties of the mass concentration solutions, such as positivity and upper boundedness. Numerical simulations of the solution of the model using the finite element method are performed in [58, 60]. The model involves the unknown moving boundary and the concentrations of involved species by means of the solution of a coupled nonlinear system of parabolic reaction-diffusion equations. As the best model may be wasted with a disregarded computation, its numerical analysis is necessary.

Other recent different model of concrete carbonation phenomenon is exposed in [45], where an initial boundary value problem is used to study carbon dioxide transport in a bounded domain of $\mathbb{R}^{3}$.

The concrete element is supposed to be a sample with thickness equal to $L$. The space variable $x$ is measured from the exposed boundary $x=0$ to the sealed boundary $x=L$, and the unknown carbonation front or moving boundary is denoted by $x=S(t)$, satisfying $0<S(t)<L$. The whole domain $[0, L]$ is divided in two subdomains, the so-called carbonated zone $\Omega_{1}(t)=[0, S(t)[$ and uncarbonated zone $\left.\left.\Omega_{2}(t)=\right] S(t), L\right]$. The chemical species present in $\Omega_{1}(t)$ are $\mathrm{CO}_{2}(\mathrm{aq})$, $\mathrm{CO}_{2}(\mathrm{~g}), \mathrm{CaCO}_{3}(\mathrm{aq})$ and $\mathrm{H}_{2} \mathrm{O}$, and the species in $\Omega_{2}(t)$ are $\mathrm{Ca}(\mathrm{OH})_{2}(\mathrm{aq})$ and $\mathrm{H}_{2} \mathrm{O}$. 
Here, (aq) and (g) refer to species in aqueous and gaseous environments, respectively. $\mathrm{CaCO}_{3}(\mathrm{aq})$ is created in the carbonation front according to the reaction

$$
\mathrm{CO}_{2}(\mathrm{aq})+\mathrm{Ca}(\mathrm{OH})_{2}(\mathrm{aq}) \longrightarrow \mathrm{CaCO}_{3}(\mathrm{aq})+\mathrm{H}_{2} \mathrm{O}
$$

and at any time $t$ is uniformly distributed in $\Omega_{1}(t)=[0, S(t)[$, [60].

The mass concentrations of the species are represented by the following variables, where time takes values in the interval $0 \leq t \leq T$,

$$
\begin{aligned}
& \bar{U}_{1}(x, t)=\left[\mathrm{CO}_{2}(a q)\right], \quad \bar{U}_{2}(x, t)=\left[\mathrm{CO}_{2}(g)\right], \quad \bar{U}_{5}(x, t)=\left[\mathrm{H}_{2} \mathrm{O}\right], \quad 0 \leq x \leq S(t), \\
& \bar{U}_{3}(x, t)=\left[\mathrm{Ca}(\mathrm{OH})_{2}(a q)\right], \quad \bar{U}_{6}(x, t)=\left[\mathrm{H}_{2} \mathrm{O}\right], \quad S(t) \leq x \leq L, \\
& \bar{U}_{4}(t)=\left[\mathrm{CaCO}_{3}(a q)\right] \text {, }
\end{aligned}
$$

where $T>0$ is the time horizon.

Throughout this chapter, we will use the notation $\bar{U}_{i}=\bar{U}_{i}(x, t), 1 \leq i \leq 6$, $i \neq 4$, and $\bar{U}_{4}=\bar{U}_{4}(t)$ for the unknown concentrations. Note that the unknown $\bar{U}_{4}$ depends only of the time because it refers to the concentration of $\mathrm{CaCO}_{3}(\mathrm{aq})$ at the unknown carbonation front $S(t)$.

The porosity of the concrete sample is given by the parameter $\phi$, while air and water fractions of the pores are denoted by $\phi_{\omega}$ and $\phi_{a}$, respectively. According to [27], it is assumed that the exchange of $\mathrm{CO}_{2}$ between the gas and liquid phases is modeled by linear Henry laws of the type

$$
f_{1, \text { Henry }}=-P_{1}\left(\phi \phi_{\omega} \bar{U}_{1}-Q_{1} \phi \phi_{a} \bar{U}_{2}\right), \quad f_{2, \text { Henry }}=P_{2}\left(\phi \phi_{\omega} \bar{U}_{1}-Q_{2} \phi \phi_{a} \bar{U}_{2}\right),
$$

where $P_{1}, P_{2}$ are mass transfer parameters through the interface air-water and $Q_{1}$, $Q_{2}$ are the positive Henry-like constants.

The dissolution rate for $\mathrm{Ca}(\mathrm{OH})_{2}(\mathrm{aq})$ is given by

$$
f_{\text {Diss }}=-S_{3, \text { diss }}\left(\bar{U}_{3}-U_{3, \text { eq }}\right)
$$

where $S_{3, \text { diss }}$ is a mass transfer coefficient and $U_{3, \mathrm{eq}}=U_{3, \mathrm{eq}}(t)$ is an equilibrium concentration, see [53, 59, 60].

The production rates of $\mathrm{CaCO}_{3}(\mathrm{aq})$ and $\mathrm{H}_{2} \mathrm{O}$ at the carbonation front are modeled by the function

$$
f_{\text {Reac } \Gamma}=\left[\kappa \phi \phi_{\omega}\left(\bar{U}_{1}\right)^{p}\left(\bar{U}_{3}\right)^{q}\right]_{\Gamma(t)},
$$




\section{6 SPECIES MODEL FOR DAMAGE BY CARBONATION OF REINFORCED CONCRETE STRUCTURES}

where

$$
\Gamma(t)=(S(t), t),
$$

and the notation $[f]_{(x, t)}=f(x, t)$ has been used. Parameter $\kappa>0$ is the reaction constant and $p \geq 1, q \geq 1$, denote the partial reaction orders. In addition, the precipitation rate of $\mathrm{CaCO}_{3}(\mathrm{aq})$ is not considered in the model, that is

$$
f_{\text {Prec }}=0 \text {. }
$$

Let $D_{i} i, \in\{1,2,3\}$ represent diffusion coefficients for each species $\bar{U}_{i}$, and let $\phi D_{5}, \phi D_{6}$ stand for transport coefficients of water content in the carbonated and uncarbonated zone, respectively. It is assumed that each $D_{i}, 1 \leq i \leq 6, i \neq 4$, is a positive constant ([59]).

The continuous model proposed in [59, 60] is described by a coupled system of five partial differential equations (PDE) and one ordinary differential equation (ODE) (balance equations); together with the initial, boundary and transmission conditions and the velocity law of the moving front. The five PDEs and the ODE involving the unknown concentrations are presented as follows:

Carbon dioxide mass concentration in water phase, $\bar{U}_{1}=\left[\mathrm{CO}_{2}(a q)\right]$ :

$$
\frac{\partial}{\partial t}\left(\phi \phi_{\omega} \bar{U}_{1}\right)-\frac{\partial}{\partial x}\left(D_{1} \phi \phi_{\omega} \frac{\partial \bar{U}_{1}}{\partial x}\right)=f_{1, \text { Henry }}, \quad 0 \leq x<S(t), \quad 0<t<T .
$$

Carbon dioxide mass concentration in air phase, $\bar{U}_{2}=\left[\mathrm{CO}_{2}(g)\right]$ :

$$
\frac{\partial}{\partial t}\left(\phi \phi_{a} \bar{U}_{2}\right)-\frac{\partial}{\partial x}\left(D_{2} \phi \phi_{a} \frac{\partial \bar{U}_{2}}{\partial x}\right)=f_{2, \text { Henry }}, \quad 0 \leq x<S(t), \quad 0<t<T .
$$

Calcium hydroxide present in the cement, $\bar{U}_{3}=\left[\mathrm{Ca}(\mathrm{OH})_{2}(a q)\right]$ :

$$
\frac{\partial}{\partial t}\left(\phi \phi_{\omega} \bar{U}_{3}\right)-\frac{\partial}{\partial x}\left(D_{3} \phi \phi_{\omega} \frac{\partial \bar{U}_{3}}{\partial x}\right)=f_{\text {Diss }}, \quad S(t)<x \leq L, \quad 0<t<T \text {. }
$$

The calcium carbonate creation in the carbonation front $\bar{U}_{4}=\left[\mathrm{CaCO}_{3}(a q)\right]$ is given by

$$
\left[\frac{d}{d t}\left(\phi \phi_{\omega} \bar{U}_{4}\right)\right]_{\Gamma(t)}=f_{\text {Prec }}+f_{\text {Reac } \Gamma}, 0<t<T
$$


Water content in the carbonated zone, $\bar{U}_{5}=\left[\mathrm{H}_{2} \mathrm{O}\right]$,

$$
\frac{\partial}{\partial t}\left(\phi \bar{U}_{5}\right)-\frac{\partial}{\partial x}\left(D_{5} \phi \frac{\partial \bar{U}_{5}}{\partial x}\right)=0, \quad 0 \leq x<S(t), \quad 0<t<T .
$$

The last balance equation for water content in the uncarbonated zone, $\bar{U}_{6}=$ $\left[\mathrm{H}_{2} \mathrm{O}\right]$, is given by

$$
\frac{\partial}{\partial t}\left(\phi \bar{U}_{6}\right)-\frac{\partial}{\partial x}\left(D_{6} \phi \frac{\partial \bar{U}_{6}}{\partial x}\right)=0, \quad S(t)<x \leq L, \quad 0<t<T .
$$

Initial position of the front is $S(0)=S_{0}>0$ and initial concentrations in their respective domains take the form

$$
\begin{gathered}
\bar{U}_{i}(x, 0)=\bar{U}_{i 0}(x), \quad 0<x<S_{0}, \quad i \in\{1,2,5\}, \\
\bar{U}_{4}(0)=\bar{U}_{40}, \\
\bar{U}_{i}(x, 0)=\bar{U}_{i 0}(x), \quad S_{0}<x<L, \quad i \in\{3,6\} .
\end{gathered}
$$

With respect to the boundary conditions at the exposed boundary $x=0$ and the sealed boundary $x=L$, one gets

$$
\begin{gathered}
\phi \phi_{\omega} \bar{U}_{1}(0, t)=\Lambda_{1}(t), \phi \phi_{a} \bar{U}_{2}(0, t)=\Lambda_{2}(t), \phi \bar{U}_{5}(0, t)=\Lambda_{5}(t), \quad 0<t<T \\
\frac{\partial \bar{U}_{3}}{\partial x}(L, t)=0, \quad \frac{\partial \bar{U}_{6}}{\partial x}(L, t)=0, \quad 0<t<T
\end{gathered}
$$

The transmission conditions at the free boundary $x=S(t), 0<t<T$, are given by

$$
\left(-\delta_{1 i}+\delta_{3 i}\right)\left[\frac{\partial}{\partial x}\left(D_{i} \phi \phi_{\omega} \bar{U}_{i}\right)\right]_{\Gamma(t)}=-\eta_{\Gamma}\left(\bar{U}_{1}, \bar{U}_{3}\right)+S^{\prime}(t)\left[\phi \phi_{\omega} \bar{U}_{i}\right]_{\Gamma(t)}, \quad i \in\{1,3\}
$$




\section{6 SPECIES MODEL FOR DAMAGE BY CARBONATION OF} REINFORCED CONCRETE STRUCTURES

$$
\begin{gathered}
-\left[\frac{\partial}{\partial x}\left(D_{2} \phi \phi_{a} \bar{U}_{2}\right)\right]_{\Gamma(t)}=S^{\prime}(t)\left[\phi \phi_{a} \bar{U}_{2}\right]_{\Gamma(t)}, \\
\left(-\delta_{5 i}+\delta_{6 i}\right)\left[\frac{\partial}{\partial x}\left(D_{i} \phi \bar{U}_{i}\right)\right]_{\Gamma(t)}=\delta_{5 i} \frac{1}{\phi_{\omega}} \eta_{\Gamma}\left(\bar{U}_{1}, \bar{U}_{3}\right)+S^{\prime}(t)\left[\phi \bar{U}_{i}\right]_{\Gamma(t)}, \quad i \in\{5,6\} .
\end{gathered}
$$

Finally, the velocity law for propagation of the carbonation front takes the form

$$
S^{\prime}(t)=\left[\alpha \kappa\left(\bar{U}_{1}\right)^{p}\left(\bar{U}_{3}\right)^{q-1}\right]_{\Gamma(t)},
$$

where $\alpha>1$ ([60], pp. 544), and the function $\eta_{\Gamma}$ is defined for a couple of functions $V_{1}(x, t)$ and $V_{3}(x, t)$ as

$$
\eta_{\Gamma}\left(V_{1}, V_{3}\right)=\kappa \phi \phi_{\omega}\left(V_{1}(S(t), t)\right)^{p}\left(V_{3}(S(t), t)\right)^{q}, \quad 0<t<T,
$$

so that $\eta_{\Gamma}\left(\bar{U}_{i}, \bar{U}_{i}\right)=f_{\text {Reac } \Gamma \text {, see } 4.5 \text {. }}$.

As the active concentrations are small, the constant porosity assumption is valid (see [9, 51, 78]), thus in this work the parameters porosity $\phi>0$ as well as water and air fractions $\phi_{\omega}>0$ and $\phi_{a}>0$ are constants.

Let us consider the suitable transformation of variables:

$$
\begin{gathered}
\hat{U}_{i}(x, t)=\phi \phi_{\omega} \bar{U}_{i}(x, t), \quad i \in\{1,3\}, \\
\hat{U}_{2}(x, t)=\phi \phi_{a} \bar{U}_{2}(x, t), \quad \hat{U}_{4}(t)=\phi \phi_{\omega} \bar{U}_{4}(t), \\
\hat{U}_{i}(x, t)=\phi \bar{U}_{i}(x, t), \quad i \in\{5,6\} .
\end{gathered}
$$

Under the new variables $\hat{U}_{i}(x, t), i \in\{1,2,3,5,6\}, \hat{U}_{4}(t)$ and using Kronecker's symbol $\delta_{i j}$, problem (4.8)-4.22 can be written in a compact form

$$
\begin{gathered}
\left(\delta_{1 i}+\delta_{2 i}+\delta_{5 i}\right) \frac{\partial \hat{U}_{i}}{\partial t}-D_{i} \frac{\partial^{2} \hat{U}_{i}}{\partial x^{2}}=\left(1-\delta_{5 i}\right)(-1)^{i} P_{i}\left(\hat{U}_{1}-Q_{i} \hat{U}_{2}\right) \\
0 \leq x<S(t), \quad 0<t<T, \quad i \in\{1,2,5\} \\
\frac{\partial \hat{U}_{i}}{\partial t}-D_{i} \frac{\partial^{2} \hat{U}_{i}}{\partial x^{2}}=\delta_{3 i}\left(-S_{3, \text { diss }}\left(\hat{U}_{3}-U_{3, \mathrm{eq}}\right)\right), \quad S(t)<x \leq L, \quad 0<t<T, \quad i \in\{3,6\},
\end{gathered}
$$




$$
\frac{d \hat{U}_{4}}{d t}=\kappa\left(\phi \phi_{\omega}\right)^{1-p-q}\left[\left(\hat{U}_{1}\right)^{p}\left(\hat{U}_{3}\right)^{q}\right]_{\Gamma(t)}, \quad 0<t<T
$$

The transformed initial conditions become $S(0)=S_{0}>0$ and

$$
\begin{gathered}
\hat{U}_{1}(x, 0)=\hat{U}_{10}(x)=\phi \phi_{\omega} \bar{U}_{10}(x), \quad \hat{U}_{2}(x, 0)=\hat{U}_{20}(x)=\phi \phi_{a} \bar{U}_{20}(x), \\
\hat{U}_{5}(x, 0)=\hat{U}_{50}(x)=\phi \bar{U}_{50}(x), 0<x<S_{0}, \\
\hat{U}_{4}(0)=\hat{U}_{40}=\phi \phi_{\omega} \bar{U}_{40}, \\
\hat{U}_{3}(x, 0)=\hat{U}_{30}(x)=\phi \phi_{\omega} \bar{U}_{30}(x), \hat{U}_{6}(x, 0)=\hat{U}_{60}(x)=\phi \bar{U}_{60}(x), \quad S_{0}<x<L,
\end{gathered}
$$

and the transformed boundary conditions are

$$
\begin{gathered}
\hat{U}_{i}(0, t)=\Lambda_{i}(t), \quad 0<t<T, \quad i \in\{1,2,5\}, \\
\frac{\partial \hat{U}_{i}}{\partial x}(L, t)=0, \quad 0<t<T, \quad i \in\{3,6\} .
\end{gathered}
$$

Finally, the transformed interface conditions for $x=S(t), 0<t<T$, become

$$
-\left[D_{i} \frac{\partial \hat{U}_{i}}{\partial x}\right]_{\Gamma(t)}=\left(\delta_{5 i}-\delta_{1 i}\right)\left(\phi \phi_{\omega}\right)^{-p-q} \eta_{\Gamma}\left(\hat{U}_{1}, \hat{U}_{3}\right)+S^{\prime}(t)\left[\hat{U}_{i}\right]_{\Gamma(t)}, \quad i \in\{1,2,5\},
$$

$$
\left[D_{i} \frac{\partial \hat{U}_{i}}{\partial x}\right]_{\Gamma(t)}=-\left(\delta_{3 i}\right)\left(\phi \phi_{\omega}\right)^{-p-q} \eta_{\Gamma}\left(\hat{U}_{1}, \hat{U}_{3}\right)+S^{\prime}(t)\left[\hat{U}_{i}\right]_{\Gamma(t)}, \quad i \in\{3,6\}
$$

and the velocity law is

$$
S^{\prime}(t)=\alpha \kappa\left(\phi \phi_{\omega}\right)^{1-p-q}\left[\left(\hat{U}_{1}\right)^{p}\left(\hat{U}_{3}\right)^{q-1}\right]_{\Gamma(t)} .
$$

In this chapter, a finite difference method to solve the moving boundary problem 4.8 -4.22 is proposed, obtaining sufficient step sizes conditions to guarantee positivity and stability of the numerical solution. The chapter is organized as 


\section{6 SPECIES MODEL FOR DAMAGE BY CARBONATION OF REINFORCED CONCRETE STRUCTURES}

follows. In Section 4.1, after a front-fixing transformation, the original problem is transformed into another one in a fixed domain, where the moving boundary becomes a new unknown of the problem. In Section 4.2, we propose a coupled finite difference scheme whose unknowns are $\left[\mathrm{CO}_{2}(\mathrm{aq})\right],\left[\mathrm{CO}_{2}(\mathrm{~g})\right],\left[\mathrm{CaCO}_{3}(\mathrm{aq})\right]$ and $\left[\mathrm{H}_{2} \mathrm{O}\right]$ in the carbonated zone, $\left[\mathrm{Ca}(\mathrm{OH})_{2}(\mathrm{aq})\right]$ and $\left[\mathrm{H}_{2} \mathrm{O}\right]$ in the uncarbonated zone, together with the square power values of the expanding front. In Section 4.3, positivity, boundedness and stability of the numerical solution is treated. Spatial monotonicity properties of the solution is addressed in Section 4.4. Experiments illustrating the qualitative properties of the numerical solution are included in the corresponding sections. The main conclusions are presented in Section 4.5.

\subsection{Front-fixing transformation}

For the sake of simplicity, and taking advance of the fact that $\left(S^{2}(t)\right)^{\prime}=2 S(t) S^{\prime}(t)$, in the following we will consider as unknown the square of the free boundary $R(t)$ instead of the free boundary itself $S(t)$ in order to obtain a more simplified PDE system, i. e.

$$
R(t)=S^{2}(t)
$$

In order to transform the PDE problem with moving domain into a fixed domain one, let us consider the following change of spatial variable inspired by the well known Landau transformation, ([26, 49]):

$$
z(x, t)= \begin{cases}(x / \sqrt{R(t)})-1, & 0 \leq x<\sqrt{R(t)}, 0 \leq t \leq T \\ 0, & x=\sqrt{R(t)}, 0 \leq t \leq T \\ (x-\sqrt{R(t)}) /(L-\sqrt{R(t)}), & \sqrt{R(t)}<x \leq L, \quad 0 \leq t \leq T .\end{cases}
$$

The new unknown variables of the problem are

$$
\begin{aligned}
& U_{i}(z, t)=\hat{U}_{i}(x, t), \quad i \in\{1,2,5\},-1 \leq z \leq 0, \\
& U_{4}(t)=\hat{U}_{4}(t), \\
& U_{i}(z, t)=\hat{U}_{i}(x, t), \quad i \in\{3,6\}, 0 \leq z \leq 1 .
\end{aligned}
$$


In a similar way as in (4.6), let us denote

$$
\gamma(t)=(0, t)
$$

Then, the new PDE system posed in the fixed domain $|z| \leq 1$ is

$$
\begin{aligned}
& \left(\delta_{1 i}+\delta_{2 i}+\delta_{5 i}\right)\left(R(t) \frac{\partial U_{i}}{\partial t}-R^{\prime}(t) \frac{z+1}{2} \frac{\partial U_{i}}{\partial z}\right)-D_{i} \frac{\partial^{2} U_{i}}{\partial z^{2}} \\
& =\left(1-\delta_{5 i}\right)(-1)^{i} P_{i}\left(U_{1}-Q_{i} U_{2}\right) R(t), \quad-1 \leq z<0, \quad 0<t<T, \quad i \in\{1,2,5\},
\end{aligned}
$$

$$
\begin{gathered}
\left(R(t)+L^{2}-2 L \sqrt{R(t)}\right) \frac{\partial U_{i}}{\partial t}+\left(\frac{R^{\prime}(t)}{2}\left(\frac{L}{\sqrt{R(t)}}-1\right)\right)(z-1) \frac{\partial U_{i}}{\partial z} \\
-D_{i} \frac{\partial^{2} U_{i}}{\partial z^{2}}=-\delta_{3 i}\left(R(t)+L^{2}-2 L \sqrt{R(t)}\right) S_{3, \text { Diss }}\left(U_{3}-U_{3, \mathrm{eq}}\right) \\
0<z \leq 1, \quad 0<t<T, \quad i \in\{3,6\} \\
\frac{d U_{4}(t)}{d t}=\kappa\left(\phi \phi_{\omega}\right)^{1-p-q}\left[\left(U_{1}\right)^{p}\left(U_{3}\right)^{q}\right]_{\gamma(t)}, \quad 0<t<T
\end{gathered}
$$

together with the initial conditions

$$
\begin{gathered}
U_{1}(z, 0)=U_{10}(z)=\phi \phi_{\omega} \bar{U}_{10}\left((z+1) S_{0}\right), \quad U_{2}(z, 0)=U_{20}(z)=\phi \phi_{a} \bar{U}_{20}\left((z+1) S_{0}\right), \\
U_{5}(z, 0)=U_{50}(z)=\phi \bar{U}_{50}\left((z+1) S_{0}\right),-1<z<0 \\
U_{4}(0)=U_{40}=\phi \phi_{\omega} \bar{U}_{40} \\
U_{3}(z, 0)=U_{30}(z)=\phi \phi_{\omega} \bar{U}_{30}\left(z\left(L-S_{0}\right)+S_{0}\right) \\
U_{6}(z, 0)=U_{60}(z)=\phi \bar{U}_{60}\left(z\left(L-S_{0}\right)+S_{0}\right), \quad 0<z<1
\end{gathered}
$$

the boundary conditions

$$
\begin{gathered}
U_{i}(-1, t)=\Lambda_{i}(t), \quad 0<t<T, \quad i \in\{1,2,5\}, \\
\frac{\partial U_{i}}{\partial z}(1, t)=0, \quad 0<t<T, \quad i \in\{3,6\},
\end{gathered}
$$




\section{6 SPECIES MODEL FOR DAMAGE BY CARBONATION OF REINFORCED CONCRETE STRUCTURES}

and the interface conditions at $z=0,0<t<T$,

$$
\begin{aligned}
& -\left[D_{i} \frac{\partial U_{i}}{\partial z}\right]_{\gamma(t)}=\left(\delta_{5 i}-\delta_{1 i}\right)\left(\phi \phi_{\omega}\right)^{-p-q} \eta_{\gamma}\left(U_{1}, U_{3}\right) \sqrt{R(t)} \\
& +\frac{1}{2} R^{\prime}(t)\left[U_{i}\right]_{\gamma(t)}, \quad i \in\{1,2,5\} \\
& {\left[D_{i} \frac{\partial U_{i}}{\partial z}\right]_{\gamma(t)}=-\left(\delta_{3 i}\right)\left(\phi \phi_{\omega}\right)^{-p-q} \eta_{\gamma}\left(U_{1}, U_{3}\right)(L-\sqrt{R(t)})} \\
& +\left(\frac{R^{\prime}(t)}{2}\left(\frac{L}{\sqrt{R(t)}}-1\right)\right)\left[U_{i}\right]_{\gamma(t)}, \quad i \in\{3,6\} \\
& R^{\prime}(t)=2 \alpha \kappa\left(\phi \phi_{\omega}\right)^{1-p-q} \sqrt{R(t)}\left[\left(U_{1}\right)^{p}\left(U_{3}\right)^{q-1}\right]_{\gamma(t)},
\end{aligned}
$$

where the function $\eta_{\gamma}$ is defined for a couple of functions $V_{1}(z, t)$ and $V_{3}(z, t)$ analogously to 4.23 .

$$
\eta_{\gamma}\left(V_{1}, V_{3}\right)=\kappa \phi \phi_{\omega}\left(V_{1}(0, t)\right)^{p}\left(V_{3}(0, t)\right)^{q}, \quad 0<t<T
$$

From (4.51) for $i=3$ and (4.52), one gets

$$
\left[D_{3} \frac{\partial U_{3}}{\partial z}\right]_{\gamma(t)}=(\alpha-1) \kappa\left(\phi \phi_{\omega}\right)^{1-p-q}\left[\left(U_{1}\right)^{p}\left(U_{3}\right)^{q}\right]_{\gamma(t)}(L-\sqrt{R(t)}) .
$$

Equation 4.54 shows that $\left[\partial U_{3} / \partial z\right]_{\gamma(t)}$ is positive. This fact will be used in Section 3 dealing with the numerical solution.

\subsection{Discretization and numerical scheme construction}

In this section we construct a finite difference scheme for solving numerically the coupled system (4.42)-(4.52). Let $M$ and $N$ be positive integers, so that the domain $[-1,1] \times[0, T]$ is partitioned in $(2 M+1) \times(N+1)$ mesh points denoted by $\left(z_{j}, t^{n}\right)$, where $z_{j}=j h,-M \leq j \leq M$ and $t^{n}=n k, 0 \leq n \leq N$. Here the step sizes 
discretizations $h$ and $k$ verify $h M=1$ and $k N=T$, respectively. Numerical approximations of the involved variables, using expressions established in Section 1.3 , are denoted by $u_{i, j}^{n} \approx U_{i}\left(z_{j}, t^{n}\right), i \in\{1,2,3,5,6\}, u_{4}^{n} \approx U_{4}\left(t^{n}\right), r^{n} \approx R\left(t^{n}\right)$, while we denote $\lambda_{i}^{n}=\Lambda_{i}\left(t^{n}\right), i \in\{1,2,5\}$.

On the assumption of the following properties

$U_{i} \in C^{2,1}([-1,0] \times[0, T]), \quad i \in\{1,2,5\} ; \quad U_{i} \in C^{2,1}([0,1] \times[0, T]), \quad i \in\{3,6\}$, $U_{4} \in C^{1}[0, T], \quad R \in C^{1}[0, T]$,

partial derivatives at the interior points are approximated using forward in time and centered in space finite difference expressions:

$$
\begin{gathered}
\frac{u_{i, j}^{n+1}-u_{i, j}^{n}}{k} \approx \frac{\partial U_{i}}{\partial t}\left(z_{j}, t^{n}\right), \quad, \frac{u_{i, j+1}^{n}-u_{i, j-1}^{n}}{2 h} \approx \frac{\partial U_{i}}{\partial z}\left(z_{j}, t^{n}\right), \\
\frac{u_{i, j+1}^{n}-2 u_{i, j}^{n}+u_{i, j-1}^{n}}{h^{2}} \approx \frac{\partial^{2} U_{i}}{\partial z^{2}}\left(z_{j}, t^{n}\right), \quad i \in\{1,2,3,5,6\}, \\
\frac{u_{4}^{n+1}-u_{4}^{n}}{k} \approx \frac{d U_{4}}{d t}\left(t^{n}\right), \frac{r^{n+1}-r^{n}}{k} \approx R^{\prime}\left(t^{n}\right) .
\end{gathered}
$$

With respect to the discretization of the first derivatives of the transformed transmission conditions at the carbonation front $z=0$, we use one side second order finite difference approximations. We take left hand side approximations with three points for the discretization at the carbonated zone:

$$
\frac{3 u_{i, 0}^{n}-4 u_{i,-1}^{n}+u_{i,-2}^{n}}{2 h} \approx \frac{\partial U_{i}}{\partial z}\left(0, t^{n}\right), \quad i \in\{1,2,5\},
$$

and the right side approximations for the discretization at the uncarbonated zone

$$
\frac{-3 u_{i, 0}^{n}+4 u_{i, 1}^{n}-u_{i, 2}^{n}}{2 h} \approx \frac{\partial U_{i}}{\partial z}\left(0, t^{n}\right), \quad i \in\{3,6\} .
$$

Using the approximations (4.56)-(4.59), equations 4.42)-(4.44) become discretized at the interior mesh points in the following way

$$
\begin{aligned}
& \left(\delta_{1 i}+\delta_{2 i}+\delta_{5 i}\right) r^{n} \frac{u_{i, j}^{n+1}-u_{i, j}^{n}}{k}-\frac{1+z_{j}}{2}\left(\frac{r^{n+1}-r^{n}}{k}\right) \frac{u_{i, j+1}^{n}-u_{i, j-1}^{n}}{2 h} \\
& -D_{i} \frac{u_{i, j+1}^{n}-2 u_{i, j}^{n}+u_{i, j-1}^{n}}{h^{2}}=\left(1-\delta_{5 i}\right)(-1)^{i} r^{n} P_{i}\left(u_{1, j}^{n}-Q_{i} u_{2, j}^{n}\right), \\
& -M+1 \leq j \leq-1, \quad 0 \leq n \leq N-1, \quad i \in\{1,2,5\},
\end{aligned}
$$




\section{6 SPECIES MODEL FOR DAMAGE BY CARBONATION OF REINFORCED CONCRETE STRUCTURES}

$$
\begin{aligned}
& \left(r^{n}+L^{2}-2 L \sqrt{r^{n}}\right) \frac{u_{i, j}^{n+1}-u_{i, j}^{n}}{k}+\frac{z_{j}-1}{2}\left(\frac{r^{n+1}-r^{n}}{k}\right)\left(\frac{L}{\sqrt{r^{n}}}-1\right) \frac{u_{i, j+1}^{n}-u_{i, j-1}^{n}}{2 h} \\
& -D_{i} \frac{u_{i, j+1}^{n}-2 u_{i, j}^{n}+u_{i, j-1}^{n}}{h^{2}}=-\delta_{3 i}\left(r^{n}+L^{2}-2 L \sqrt{r^{n}}\right) S_{3, \mathrm{diss}}\left(u_{3, j}^{n}-u_{3, \mathrm{eq}}^{n}\right),
\end{aligned}
$$

$1 \leq j \leq M, \quad 0 \leq n \leq N-1, \quad i \in\{3,6\}$.

In equation 4.61, for $j=M$, it appears the concentrations $u_{i, M+1}^{n}, i \in\{3,6\}$, corresponding to the mesh point $z_{M+1}=(M+1) h$ out of the numerical domain. To find the value of $u_{i, M+1}^{n}$, the finite difference approximations of the boundary conditions at the sealed surface are used as follows, see 4.49,

$$
\frac{u_{i, M+1}^{n}-u_{i, M-1}^{n}}{2 h}=0, \quad 0 \leq n \leq N, \quad i \in\{3,6\}
$$

obtaining $u_{i, M+1}^{n}=u_{i, M-1}^{n}$.

The discretization of the ODE (4.44) takes the form

$$
\frac{u_{4}^{n+1}-u_{4}^{n}}{k}=\kappa\left(\phi \phi_{\omega}\right)^{1-p-q}\left(u_{1,0}^{n}\right)^{p}\left(u_{3,0}^{n}\right)^{q}, \quad 0 \leq n \leq N-1 .
$$

Initial concentrations given in 4.45)-4.47) take the discrete form

$$
\begin{gathered}
u_{1, j}^{0}=U_{10}\left(z_{j}\right)=\phi \phi_{\omega} \bar{U}_{10}\left(z_{j} S_{0}\right), \quad u_{2, j}^{0}=U_{20}\left(z_{j}\right)=\phi \phi_{a} \bar{U}_{20}\left(z_{j} S_{0}\right) \\
u_{5, j}^{0}=U_{50}\left(z_{j}\right)=\phi \bar{U}_{50}\left(z_{j} S_{0}\right), \quad-M+1 \leq j \leq-1, \\
u_{4}^{0}=U_{40}=\phi \phi_{\omega} \bar{U}_{40}, \\
\left.u_{3, j}^{0}=U_{30}\left(z_{j}\right)=\phi \phi_{\omega} \bar{U}_{30}\left(z_{j} S_{0}\right), \quad u_{6, j}^{0}=U_{60}\left(z_{j}\right)=\phi \bar{U}_{60}\left(z_{j} S_{0}\right), \quad 1 \leq j \leq M .64\right)
\end{gathered}
$$

Boundary conditions at the exposed surface take the values

$$
u_{i,-M}^{n}=\lambda_{i}^{n}, \quad 0 \leq n \leq N, \quad i \in\{1,2,5\} .
$$

The discretization of the Stefan-like conditions 4.50-4.52) takes the form

$$
\begin{aligned}
& -D_{i} \frac{3 u_{i, 0}^{n}-4 u_{i,-1}^{n}+u_{i,-2}^{n}}{2 h}=\left(\delta_{5 i}-\delta_{1 i}\right) \kappa\left(\phi \phi_{\omega}\right)^{1-p-q} \sqrt{r^{n}}\left(u_{1,0}^{n}\right)^{p}\left(u_{3,0}^{n}\right)^{q} \\
& +\frac{1}{2} \frac{r^{n+1}-r^{n}}{k} u_{i, 0}^{n}, \quad 0 \leq n \leq N-1, \quad i \in\{1,2,5\}
\end{aligned}
$$




$$
\begin{aligned}
& -D_{i} \frac{3 u_{i, 0}^{n}-4 u_{i, 1}^{n}+u_{i, 2}^{n}}{2 h}=-\delta_{3 i} \kappa\left(\phi \phi_{\omega}\right)^{1-p-q}\left(L-\sqrt{r^{n}}\right)\left(u_{1,0}^{n}\right)^{p}\left(u_{3,0}^{n}\right)^{q} \\
& +\frac{1}{2} \frac{r^{n+1}-r^{n}}{k}\left(\frac{L}{\sqrt{r^{n}}}-1\right) u_{i, 0}^{n}, \quad 0 \leq n \leq N-1, \quad i \in\{3,6\}
\end{aligned}
$$

and the discretization of the propagation velocity of the carbonation front

$$
\frac{r^{n+1}-r^{n}}{k}=2 \alpha \kappa\left(\phi \phi_{\omega}\right)^{1-p-q} \sqrt{r^{n}}\left(u_{1,0}^{n}\right)^{p}\left(u_{3,0}^{n}\right)^{q-1}, \quad 0 \leq n \leq N-1 .
$$

Equations (4.60) and (4.61) provide the numerical solution at the interior points. The values at the natural boundaries (exposed $z=-1$ and sealed $z=1$ ) are given by (4.67) and (4.62), respectively.

With respect to the unknown concentrations at the interface boundary $z=0$ at each temporal step $n, u_{i, 0}^{n}, i \in\{1,2,3,5,6\}$, the driving equations take the form (4.68), 4.69) and (4.70), building a nonlinear system involving also the discretized transformed interface boundary $r^{n+1}$. Note that, from (4.68) and 4.69, the unknown $u_{i, 0}^{n}, 1 \leq i \leq 6, i \neq 4$ depend on three unknown $r^{n+1}, u_{1,0}^{n}$ and $u_{3,0}^{n}$, i. e. $u_{i, 0}^{n}=\xi_{i}\left(r^{n+1}, u_{1,0}^{n}, u_{3,0}^{n}\right)$. Also, from 4.70, $r^{n+1}$ depends on $u_{1,0}^{n}$ and $u_{3,0}^{n}$. Thus, $r^{n+1}=\psi\left(u_{1,0}^{n}, u_{3,0}^{n}\right)$. Hence, let us consider the subsystem of three equations (4.68) fixing $i=1$, 4.69) fixing $i=3$ and (4.70), whose unknows are $u_{1,0}^{n}, u_{3,0}^{n}$ and $r^{n+1}$. After straightforward manipulations of these equations, one gets firstly $u_{3,0}^{n}$ from

$$
\begin{aligned}
& h^{\frac{1}{p}} \sqrt{r^{n}}\left(\left(L-\sqrt{r^{n}}\right)(\alpha-1)\right)^{\frac{1}{p}} \frac{D_{3}}{2}\left(-3 u_{3,0}^{n}+4 u_{3,1}^{n}-u_{3,2}^{n}\right)\left(u_{3,0}^{n}\right)^{\frac{p+q}{p}} \\
& +h^{\frac{1}{p}}\left(\left(L-\sqrt{r^{n}}\right)(\alpha-1)\right)^{\frac{p+1}{p}} \frac{D_{1}}{2}\left(4 u_{1,-1}^{n}-u_{1,-2}^{n}\right)\left(u_{3,0}^{n}\right)^{\frac{p+q}{p}} \\
& -\left(L-\sqrt{r^{n}}\right)(\alpha-1) \kappa^{-\frac{1}{p}}\left(\phi \phi_{\omega}\right)^{\frac{p+q-1}{p}} \frac{3 D_{1}}{2}\left(\frac{D_{3}}{2}\left(-3 u_{3,0}^{n}+4 u_{3,1}^{n}-u_{3,2}^{n}\right)\right)^{\frac{1}{p}} u_{3,0}^{n} \\
& -\sqrt{r^{n}} \alpha \kappa^{-\frac{1}{p}}\left(\phi \phi_{\omega}\right)^{\frac{p+q-1}{p}}\left(\frac{D_{3}}{2}\left(-3 u_{3,0}^{n}+4 u_{3,1}^{n}-u_{3,2}^{n}\right)\right)^{\frac{p+1}{p}}=0, \quad 0 \leq n \leq N-1 .
\end{aligned}
$$

Note that expression (4.71) is well defined when $u_{3,0}^{n}$ is positive and the basis of the powers arising in (4.71) are also positive. Since $\alpha>1$ and the carbonation 


\section{6 SPECIES MODEL FOR DAMAGE BY CARBONATION OF REINFORCED CONCRETE STRUCTURES}

front $\sqrt{r^{n}}$ is on the left of the sealed boundary $z=1$, one gets that coefficient $\left(L-\sqrt{r^{n}}\right)(\alpha-1)$ is positive. Otherwise, from 4.59 for $i=3$, and positivity of the partial derivative $\left[\partial U_{3} / \partial z\right]_{\gamma(t)}$ in $(4.54)$, it holds that $\left(-3 u_{3,0}^{n}+4 u_{3,1}^{n}-u_{3,2}^{n}\right)$ is positive for small enough values of $h$.

The discussion of existence and uniqueness of solution of (4.71) will be treated at the end of this section.

Once the value $u_{3,0}^{n}$ is found, $u_{1,0}^{n}$ is calculated using (4.68) for $i=1$ and 4.70 by

$$
\begin{aligned}
& u_{1,0}^{n}=\left\{D_{3} \frac{-3 u_{3,0}^{n}+4 u_{3,1}^{n}-u_{3,2}^{n}}{2 h}\left(\kappa\left(\phi \phi_{\omega}\right)^{1-p-q}\left(u_{3,0}^{n}\right)^{q}\left(L-\sqrt{r^{n}}\right)(\alpha-1)\right)^{-1}\right\}^{\frac{1}{p}}, \\
& 0 \leq n \leq N-1,
\end{aligned}
$$

and for the discretized free boundary value $r^{n+1}$ one gets

$$
r^{n+1}=r^{n}+2 k \alpha \kappa\left(\phi \phi_{\omega}\right)^{1-p-q} \sqrt{r^{n}}\left(u_{1,0}^{n}\right)^{p}\left(u_{3,0}^{n}\right)^{q-1}, \quad 0 \leq n \leq N-1 .
$$

Note that carbonation front $r^{n}$ is time increasing while $u_{3,0}^{n}$ be positive, because in such situation $u_{1,0}^{n}$ will be also positive.

Then, values $u_{1,0}^{n}, u_{3,0}^{n}$ and $r^{n+1}$ are obtained, the solution of system 4.68(4.69) provides the values of the remaining unknowns $u_{i, 0}^{n}, i=2,5,6$. From (4.68), for $i=2$

$$
u_{2,0}^{n}=\frac{D_{2}}{2 h}\left(4 u_{2,-1}^{n}-u_{2,-2}^{n}\right)\left(\frac{1}{2} \frac{r^{n+1}-r^{n}}{k}+\frac{3 D_{2}}{2 h}\right)^{-1}, 0 \leq n \leq N-1 .
$$

From (4.68), for $i=5$

$$
\begin{aligned}
& u_{5,0}^{n}=\left(\frac{D_{5}}{2 h}\left(4 u_{5,-1}^{n}-u_{5,-2}^{n}\right)-\kappa\left(\phi \phi_{\omega}\right)^{1-p-q} \sqrt{r^{n}}\left(u_{1,0}^{n}\right)^{p}\left(u_{3,0}^{n}\right)^{q}\right) \\
& \left(\frac{1}{2} \frac{r^{n+1}-r^{n}}{k}+\frac{3 D_{5}}{2 h}\right)^{-1}, 0 \leq n \leq N-1 .
\end{aligned}
$$

And finally, from (4.69), for $i=6$

$$
u_{6,0}^{n}=\frac{D_{6}}{2 h}\left(4 u_{6,1}^{n}-u_{6,2}^{n}\right)\left(\frac{1}{2} \frac{r^{n+1}-r^{n}}{k}\left(\frac{L}{\sqrt{r^{n}}}-1\right)+\frac{3 D_{6}}{2 h}\right)^{-1}, 0 \leq n \leq N-1 .
$$


From equations (4.60)-4.61) the solutions at the interior points at time level $n+1$ are given by:

$$
\begin{aligned}
& u_{i, j}^{n+1}=a_{i, j}^{n} u_{i, j-1}^{n}+b_{i, j}^{n} u_{i, j}^{n}+c_{i, j}^{n} u_{i, j+1}^{n}+\delta_{1 i} k P_{1} Q_{1} u_{2, j}^{n}+\delta_{2 i} k P_{2} u_{1, j}^{n}, \\
& -M+1 \leq j \leq-1, \quad 0 \leq n \leq N-1, \quad i \in\{1,2,5\}, \\
& u_{i, j}^{n+1}=a_{i, j}^{n} u_{i, j-1}^{n}+b_{i, j}^{n} u_{i, j}^{n}+c_{i, j}^{n} u_{i, j+1}^{n}+\delta_{3 i} k S_{3, \text { diss }} u_{3, \mathrm{eq}}^{n}, \\
& 1 \leq j \leq M, \quad 0 \leq n \leq N-1, \quad i \in\{3,6\},
\end{aligned}
$$

where

$$
\begin{gathered}
a_{i, j}^{n}= \begin{cases}\frac{D_{i} k}{h^{2} r^{n}}-\frac{1+z_{j}}{4 h} \Delta_{1}^{n}, & i \in\{1,2,5\}, \\
\frac{D_{i} k}{h^{2} \Delta_{3}^{n}}+\frac{z_{j}-1}{4 h} r^{n} \frac{\Delta_{1}^{n} \Delta_{2}^{n}}{\Delta_{3}^{n}}, & i \in\{3,6\},\end{cases} \\
b_{i, j}^{n}= \begin{cases}1-\frac{2 D_{i} k}{h^{2} r^{n}}-\delta_{1 i} k P_{1}-\delta_{2 i} k P_{2} Q_{2}, & i \in\{1,2,5\}, \\
1-\frac{2 D_{i} k}{h^{2} \Delta_{3}^{n}}-\delta_{3 i} k S_{3, \text { diss }}, & i \in\{3,6\},\end{cases} \\
c_{i, j}^{n}= \begin{cases}\frac{D_{i} k}{h^{2} r^{n}}+\frac{1+z_{j}}{4 h} \Delta_{1}^{n}, & i \in\{1,2,5\}, \\
\frac{D_{i} k}{h^{2} \Delta_{3}^{n}}+\frac{1-z_{j}}{4 h} r^{n} \frac{\Delta_{1}^{n} \Delta_{2}^{n}}{\Delta_{3}^{n}}, & i \in\{3,6\},\end{cases}
\end{gathered}
$$

and

$$
\Delta_{1}^{n}=\frac{r^{n+1}}{r^{n}}-1, \quad \Delta_{2}^{n}=\frac{L}{\sqrt{r^{n}}}-1, \quad \Delta_{3}^{n}=\left(L-\sqrt{r^{n}}\right)^{2}, \quad 0 \leq n \leq N-1 .
$$

Finally, from 4.63), the concentration of $\mathrm{CaCO}_{3}(\mathrm{aq})$ in the carbonation front at the step $n+1$ is given by

$$
u_{4}^{n+1}=u_{4}^{n}+k \kappa\left(\phi \phi_{\omega}\right)^{1-p-q}\left(u_{1,0}^{n}\right)^{p}\left(u_{3,0}^{n}\right)^{q}, \quad 0 \leq n \leq N-1 .
$$

We conclude this Section with the solvability for $u_{3,0}^{n}$ of the nonlinear equation (4.71). Note that this equation (4.71) can be rewritten in the compact form

$$
F_{n}(\xi)=A_{n}\left(B_{n}-3 \xi\right) \xi^{\frac{p+q}{p}}+C_{n} \xi^{\frac{p+q}{p}}-D_{n}\left(B_{n}-3 \xi\right)^{\frac{1}{p}} \xi-E_{n}\left(B_{n}-3 \xi\right)^{\frac{p+1}{p}}=0 .
$$




\section{6 SPECIES MODEL FOR DAMAGE BY CARBONATION OF REINFORCED CONCRETE STRUCTURES}

Here, coefficients of equation (4.84) are given by

$$
\begin{aligned}
& K_{1, n}=\left(L-\sqrt{r^{n}}\right)(\alpha-1), \quad K_{2, n}=4 u_{3,1}^{n}-u_{3,2}^{n}, \quad K_{3, n}=4 u_{1,-1}^{n}-u_{1,-2}^{n}, \\
& A_{n}=h^{\frac{1}{p}} \sqrt{r^{n}}\left(K_{1, n}\right)^{\frac{1}{p}} \frac{D_{3}}{2}, \quad B_{n}=K_{2, n}, \quad C_{n}=h^{\frac{1}{p}}\left(K_{1, n}\right)^{\frac{p+1}{p}} K_{3, n} \frac{D_{1}}{2} \\
& D_{n}=K_{1, n} \kappa^{-\frac{1}{p}}\left(\phi \phi_{\omega}\right)^{\frac{p+q-1}{p}} \frac{3 D_{1}}{2}\left(\frac{D_{3}}{2}\right)^{\frac{1}{p}}, \quad E_{n}=\alpha \sqrt{r^{n}} \kappa^{-\frac{1}{p}}\left(\phi \phi_{\omega}\right)^{\frac{p+q-1}{p}}\left(\frac{D_{3}}{2}\right)^{\frac{p+1}{p}} .
\end{aligned}
$$

From the positivity of the involved coefficients of the expression of $E_{n}$ given in (4.85), it is clear that $E_{n}>0$. Since $\alpha>1$ and the carbonation front $\sqrt{r^{n}}$ is on the left of the sealed boundary $x=L$, one gets that coefficient $K_{1, n}$ is positive. Hence, coefficients $A_{n}$ and $D_{n}$ are also positive. Positivity of coefficients $K_{2, n}$ and $K_{3, n}$ can be proved in a similar way; for the sake of brevity we will state just that $K_{2, n}>0$ for small enough values of $h$. In fact, from the continuous spatial differenciability of the theoretical exact solution $U_{3}(z, t)$ ([59], Theorem 3.6) and the mean value theorem, one gets

$$
u_{3,2}^{n}=u_{3,1}^{n}+h \frac{\partial U_{3}}{\partial z}\left(\xi, t^{n}\right) ; \quad h<\xi<2 h .
$$

Let $e_{n}$ be defined by

$$
e_{n}=\max \left|\frac{\partial U_{3}}{\partial z}\left(z, t^{n}\right)\right|, \quad 0 \leq z \leq 1
$$

from 4.86) and 4.87)

$$
\left|u_{3,2}^{n}-u_{3,1}^{n}\right|<e_{n} h
$$

Hence, taking $h<3 u_{3,1} / e_{n}$, one gets

$$
K_{2, n}=4 u_{3,1}^{n}-u_{3,2}^{n}=3 u_{3,1}^{n}+\left(u_{3,1}^{n}-u_{3,2}^{n}\right)>3 u_{3,2}^{n}-e_{n} h>0 .
$$

The positivity of $K_{3, n}$ for small enough values of $h$ can be stated in an analogous way.

Hence, the remaining coefficients $B_{n}$ and $C_{n}$ become also positive for small enough values of $h$. The function $F_{n}(\xi)$ given by 4.84 is well defined, continuous 
and differentiable as a function of $\xi$ in the interval $0 \leq \xi \leq B_{n} / 3$ and its sign changes in both extremes:

$$
F_{n}(0)=-E_{n}\left(B_{n}\right)^{\frac{p+1}{p}}<0, \quad F_{n}\left(B_{n} / 3\right)=C_{n}\left(B_{n} / 3\right)^{\frac{p+q}{p}}>0 .
$$

Thus, the equation (4.84) admits a solution and now we will prove that the solution is unique under appropriate conditions. Taking derivatives in the expression of $F_{n}(\xi)$ and using that $0 \leq \xi \leq B_{n} / 3$ one gets

$$
\begin{aligned}
& F_{n}^{\prime}(\xi)=-3 A_{n}\left(\frac{2 p+q}{p}\right) \xi^{\frac{p+q}{p}}+\left(A_{n} B_{n}+C_{n}\right)\left(\frac{p+q}{p}\right) \xi^{\frac{q}{p}} \\
& +\left(3 E_{n}\left(\frac{p+1}{p}\right)-D_{n}\right)\left(B_{n}-3 \xi\right)^{\frac{1}{p}}+\frac{3 D_{n}}{p} \xi\left(B_{n}-3 \xi\right)^{\frac{1-p}{p}} \\
& \geq-3 A_{n}\left(\frac{2 p+q}{p}\right) \frac{B_{n}}{3} \xi^{\frac{q}{p}}+\left(A_{n} B_{n}+C_{n}\right)\left(\frac{p+q}{p}\right) \xi^{\frac{q}{p}} \\
& +\left(3 E_{n}\left(\frac{p+1}{p}\right)-D_{n}\right)\left(B_{n}-3 \xi\right)^{\frac{1}{p}}+\frac{3 D_{n}}{p} \xi\left(B_{n}-3 \xi\right)^{\frac{1-p}{p}} \\
& =\left(-A_{n} B_{n}\left(\frac{2 p+q}{p}\right)+\left(A_{n} B_{n}+C_{n}\right)\left(\frac{p+q}{p}\right)\right) \xi^{\frac{q}{p}} \\
& +\left(3 E_{n}\left(\frac{p+1}{p}\right)-D_{n}\right)\left(B_{n}-3 \xi\right)^{\frac{1}{p}}+\frac{3 D_{n}}{p} \xi\left(B_{n}-3 \xi\right)^{\frac{1-p}{p}} .
\end{aligned}
$$

Note that if

$$
\left(A_{n} B_{n}+C_{n}\right)\left(\frac{p+q}{p}\right) \geq A_{n} B_{n}\left(\frac{2 p+q}{p}\right), 3 E_{n}\left(\frac{p+1}{p}\right) \geq D_{n}
$$

then the nonnegativity of the derivative $F_{n}^{\prime}(\xi)$ is guaranteed.

Taking into account (4.85), note that conditions (4.92) hold true when

$$
\rho_{1} \leq \frac{\sqrt{r^{n}}}{L} \leq \rho_{2, n}
$$

where

$$
\rho_{1}=1-\frac{\alpha}{(\alpha-1)} \frac{D_{3}}{D_{1}}\left(\frac{p+1}{p}\right), \rho_{2, n}=1-\frac{1}{(\alpha-1)} \frac{D_{3}}{D_{1}}\left(\frac{p}{p+q}\right) \frac{K_{2, n}}{K_{3, n}} .
$$

Note that this means that $u_{3,0}^{n}$ is uniquely determined and positive while the carbonation front $\sqrt{r^{n}}$ satisfies condition 4.93 , i. e., when the carbonation front lies in the set $\left[\rho_{1} L, \rho_{2, n} L\right] \cap[0, L]$. 


\section{6 SPECIES MODEL FOR DAMAGE BY CARBONATION OF REINFORCED CONCRETE STRUCTURES}

As we wrote above, see (4.73), the positivity of $u_{3,0}^{n}$ guarantees that the carbonation front is time increasing, as it is expected.

We can summarize the construction of the numerical solution in the procedure exposed in Algorithm 2.

\subsection{Numerical analysis: stability and positivity}

Dealing with concentrations, the numerical solution of the scheme 4.77)-4.83 together with the values at the boundaries have to be positive. Apart from the positivity, it is convenient to study the stability of the numerical solution. Thus, the numerical solution of our scheme will preserve the qualitative properties of the theoretical solution proved in [59], Theorem 3.3.

In this section, we assume the hypothesis of the continuous model about the bounds of initial and boundary concentrations of $\mathrm{CO}_{2}(\mathrm{aq})$ and $\mathrm{CO}_{2}(\mathrm{~g})$ relationships,

$$
Q_{1} \tilde{G}_{2} \leq \tilde{G}_{1}, \quad \tilde{G}_{1} \leq Q_{2} \tilde{G}_{2}
$$

where $\tilde{G}_{1}$ is the upper bound of carbon dioxide mass concentration in water phase, and $\tilde{G}_{2}$ is the upper bound of carbon dioxide mass concentration in air phase, for both at the exposed boundary in any time and in the carbonated zone at the initial time, together with the condition on the equilibrium concentration of calcium hydroxide,

$$
u_{3, \mathrm{eq}}^{n} \leq \tilde{G}_{3},
$$

see [59], Section 3, pp. 240. Here $Q_{1}$ and $Q_{2}$ are introduced in expression (4.3).

In addition, we will assume the existence of an upper bound $\tilde{G}_{5}$ for the water content for both at the exposed boundary in any time and in the carbonated region at the initial time. Regarding the uncarbonated zone, we will suppose that mass concentration of $\mathrm{Ca}(\mathrm{OH})_{2}(\mathrm{aq})$ and water content are upper-bounded by $\tilde{G}_{3}$ and $\tilde{G}_{6}$, respectively, at the initial time, see [59], Section 3, pp. 239-240.

Let $U_{i 0}\left(z_{j}\right), 1 \leq i \leq 6, i \neq 4$, be the initial conditions given by (4.64)-(4.66) and let $\lambda_{i}^{n}, i \in\{1,2,5\}$, be the exposed boundary conditions given by (4.67). Then, according to the above hypotheses, one can write

$$
\lambda_{i}^{n} \leq \tilde{G}_{i}, \quad i \in\{1,2,5\}, \quad 0 \leq n \leq N,
$$




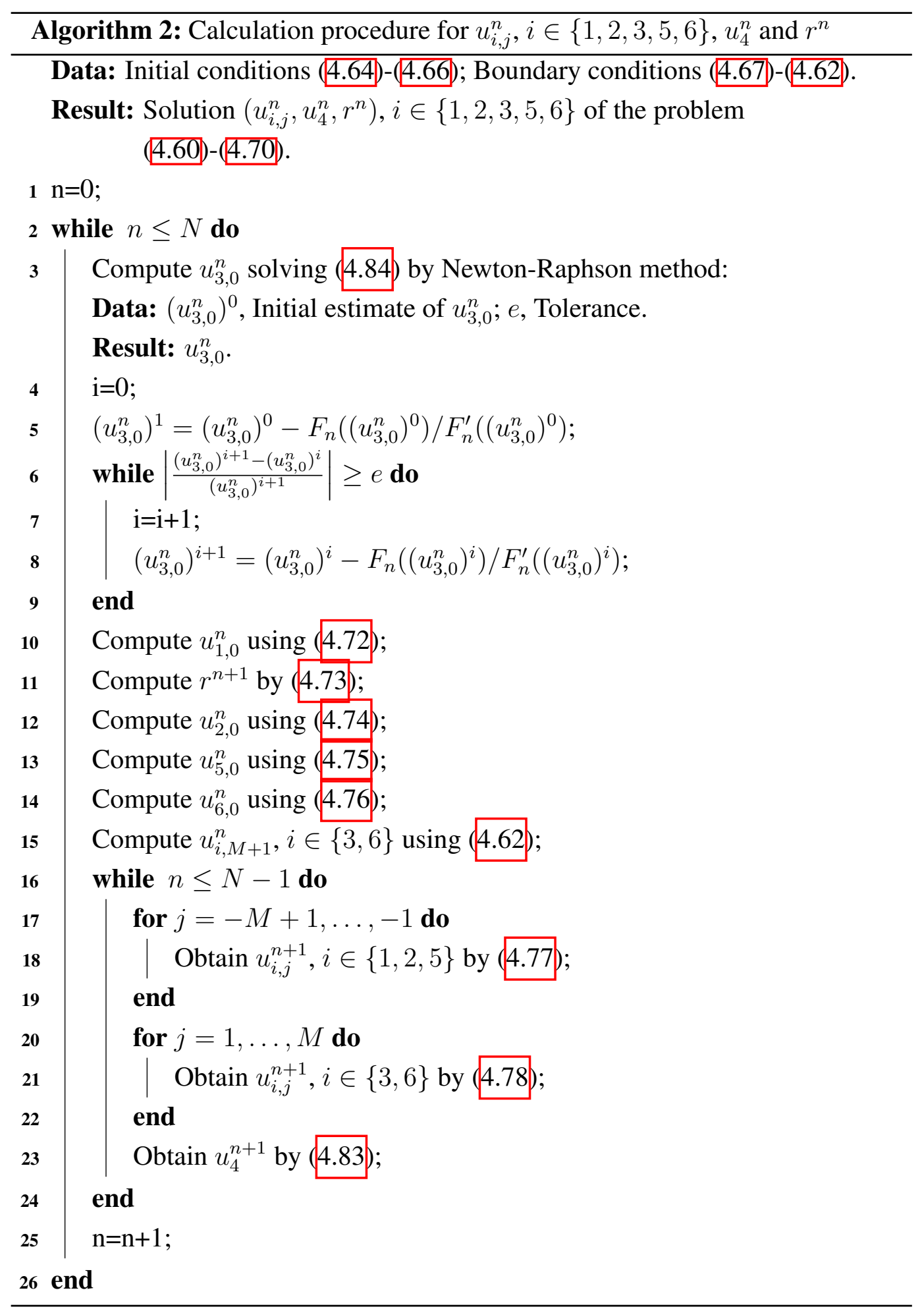




\section{6 SPECIES MODEL FOR DAMAGE BY CARBONATION OF REINFORCED CONCRETE STRUCTURES}

and

$$
\begin{aligned}
& u_{i, j}^{0}=U_{i 0}\left(z_{j}\right) \leq \tilde{G}_{i}, \quad-M+1 \leq j \leq-1, \quad, i \in\{1,2,5\}, \\
& u_{i, j}^{0}=U_{i 0}\left(z_{j}\right) \leq \tilde{G}_{i}, \quad 1 \leq j \leq M, \quad i \in\{3,6\} .
\end{aligned}
$$

We will find sufficient conditions on the discretization step sizes $h$ and $k$ under which the numerical solution $\left\{u_{i, j}^{n}\right\}$ and $\left\{u_{4}^{n}\right\}$ of the scheme 4.77-4.83 is positive and bounded. The results are obtained using the induction principle on the temporal index $n$, uniformly on the spatial index $j$.

Firstly, let us derive some numerical results based on the approximation of the spatial partial derivatives of the concentrations at the carbonation front. The righthand side approximation of the spatial partial derivative of the concentrations of chemical species in the carbonated zone $U_{i}(z, t)$ at $\left(0, t^{n}\right)$ can be written in the form

$$
\frac{-3 u_{i, 0}^{n}+4 u_{i, 1}^{n}-u_{i, 2}^{n}}{2 h}=\frac{\partial U_{i}}{\partial z}\left(0, t^{n}\right)+\mathcal{O}\left(h^{2}\right), \quad i \in\{1,2,5\},
$$

where the artificial values $u_{i, 1}^{n}$ and $u_{i, 2}^{n}$ vanish for $0 \leq n \leq N$ and $i \in\{1,2,5\}$, because they are outside of the real carbonated region.

Otherwise, the left-hand side backward approximation of the spatial partial derivative behaves, according to 4.58,

$$
\frac{3 u_{i, 0}^{n}-4 u_{i,-1}^{n}+u_{i,-2}^{n}}{2 h}=\frac{\partial U_{i}}{\partial z}\left(0, t^{n}\right)+\mathcal{O}\left(h^{2}\right), \quad i \in\{1,2,5\} .
$$

From (4.99)-4.100), one gets

$$
6 u_{i, 0}^{n}=4 u_{i,-1}^{n}-u_{i,-2}^{n}+\mathcal{O}\left(h^{3}\right), \quad i \in\{1,2,5\} .
$$

On the other hand, let us consider the left-hand side approximation of the spatial partial derivative of $U_{i}(z, t)$ at $\left(0, t^{n}\right)$

$$
\frac{3 u_{i, 0}^{n}-4 u_{i,-1}^{n}+u_{i,-2}^{n}}{2 h}=\frac{\partial U_{i}}{\partial z}\left(0, t^{n}\right)+\mathcal{O}\left(h^{2}\right), \quad i \in\{3,6\}
$$

where the artificial values $u_{i,-1}^{n}$ and $u_{i,-2}^{n}$ vanish.

The right-hand side backward approximation of the spatial partial derivative is, according to (4.59)

$$
\frac{-3 u_{i, 0}^{n}+4 u_{i, 1}^{n}-u_{i, 2}^{n}}{2 h}=\frac{\partial U_{i}}{\partial z}\left(0, t^{n}\right)+\mathcal{O}\left(h^{2}\right), \quad i \in\{3,6\},
$$




\subsection{Numerical analysis: stability and positivity}

and from (4.102)-(4.103), it follows that

$$
6 u_{i, 0}^{n}=4 u_{i, 1}^{n}-u_{i, 2}^{n}+\mathcal{O}\left(h^{3}\right), \quad i \in\{3,6\} .
$$

Equations 4.101-4.104 will be used in the study of the boundedness of $\left\{u_{i, 0}^{n}\right\}$ for both indexes 0 and $n$.

For $n=0$, initial concentrations $u_{i, j}^{0}, 1 \leq i \leq 6, i \neq 4,-M \leq j \leq M, j \neq 0$, and $u_{4}^{0} \geq 0$ are given and non negative. From the results of Section 3 , one gets the positivity of $u_{i, 0}^{0}, 1 \leq i \leq 6, i \neq 4$. Using 4.73 for $n=0$, the transformed carbonation front verifies $r^{1}>r^{0}>0$. Let $\tilde{G}_{i}$ be the positive bounds, such that

$$
0 \leq u_{i, j}^{0} \leq \tilde{G}_{i}, \quad 1 \leq i \leq 6, \quad i \neq 4
$$

Using (4.101, 4.104) and (4.105), one gets

$$
\begin{aligned}
& u_{i, 0}^{0} \leq \frac{2}{3} u_{i,-1}^{0} \leq \tilde{G}_{i}, \quad i \in\{1,2,5\}, \\
& u_{i, 0}^{0} \leq \frac{2}{3} u_{i, 1}^{0} \leq \tilde{G}_{i}, \quad i \in\{3,6\} .
\end{aligned}
$$

Let us assume the induction hypothesis, i. e., concentrations $u_{i, j}^{n}$ satisfy

$$
0 \leq u_{i, j}^{n} \leq \tilde{G}_{i}, \quad-M \leq j \leq M, \quad 1 \leq i \leq 6, \quad i \neq 4
$$

The behaviour of concentration $u_{4}^{n}$ is different and will be treated later and separately. Note that from 4.77)-4.78, the numerical solution $u_{i, j}^{n+1}$ at the points $-M+1 \leq j \leq M$ is guaranteed to be non negative if coefficients $a_{i, j}^{n}, b_{i, j}^{n}$ and $c_{i, j}^{n}$ are non negative.

The coefficients $c_{i, j}^{n}$ in equations (4.77)-(4.78) and (4.81) are unconditionally positive. The nonnegativity of the coefficients $a_{i, j}^{n}, i \in\{1,2,5\}$, in equations (4.77)-(4.78) and 4.79) will be proved using the value of the difference $r^{n+1}-r^{n}$ that appears in the transmission conditions (4.68). Using the induction principle, positivity of $u_{3,0}^{n}$ and $u_{1,0}^{n}$ and equations 4.72 - 4.73, one gets that $r^{n+1}>r^{n}>$ $r^{0}>0$.

Using (4.68) and (4.101) for $i=1$, one gets

$$
r^{n+1}-r^{n}=3 D_{1} \frac{k}{h}+2 k \kappa\left(\phi \phi_{\omega}\right)^{1-p-q} \sqrt{r^{n}}\left(u_{1,0}^{n}\right)^{p-1}\left(u_{3,0}^{n}\right)^{q}+\mathcal{O}\left(k h^{2}\right),
$$




\section{6 SPECIES MODEL FOR DAMAGE BY CARBONATION OF REINFORCED CONCRETE STRUCTURES}

and from (4.77) and (4.82), taking into account that $-1<z_{j}<0$, one gets

$$
a_{1, j}^{n}>\frac{k}{h^{2}}\left(\frac{D_{1}}{4 r^{n}}-\frac{h}{2} \kappa\left(\phi \phi_{\omega}\right)^{1-p-q} \frac{\left(u_{1,0}^{n}\right)^{p-1}\left(u_{3,0}^{n}\right)^{q}}{\sqrt{r^{n}}}+\mathcal{O}\left(h^{3}\right)\right) .
$$

Note that the time horizon $T$ is chosen so that the carbonation front does not reach the sealed boundary, $L-\sqrt{r^{n}}>0, T=N k, 0 \leq n \leq N$. At any time $0 \leq t^{n} \leq T, 0 \leq n \leq N$, the carbonation front takes the value $\sqrt{r^{n}}$ between the initial and the final position. This value $\sqrt{r^{n}}$ does not reach the sealed boundary. Thus, there exists a positive number $\beta$ such that $\sqrt{r^{0}} / L<\beta<1$, verifying

$$
0<\sqrt{r^{0}}<\sqrt{r^{n}}<\sqrt{r^{N}} \leq \beta L<L
$$

Hence, using the induction hypothesis 4.107) for $i=1, i=3$ and $j=0$, and expression 4.110, coefficients $a_{1, j}^{n}$ in equation 4.109) are positive for $-M+1 \leq$ $j \leq-1$, with the condition on the spatial step size

$$
h_{1}<\frac{D_{1}}{2 \beta L \kappa\left(\phi \phi_{\omega}\right)^{1-p-q}\left(\tilde{G}_{1}\right)^{p-1}\left(\tilde{G}_{3}\right)^{q}} .
$$

For $i=2$, using (4.68) and (4.101), it follows that

$$
r^{n+1}-r^{n}=3 D_{2} \frac{k}{h}+\mathcal{O}\left(k h^{2}\right),
$$

and from (4.77) and (4.82), using that $-1<z_{j}<0$,

$$
a_{2, j}^{n}>\frac{k}{h^{2}}\left(\frac{D_{2}}{4 r^{n}}+\mathcal{O}\left(h^{3}\right)\right),
$$

and coefficients $a_{2, j}^{n}$ are positive for $-M+1 \leq j \leq-1$.

And finally, for $i=5$, using (4.68) and (4.101), it follows that

$$
r^{n+1}-r^{n}=3 D_{5} \frac{k}{h}-2 k \kappa\left(\phi \phi_{\omega}\right)^{1-p-q} \sqrt{r^{n}} \frac{\left(u_{1,0}^{n}\right)^{p}\left(u_{3,0}^{n}\right)^{q}}{u_{5,0}^{n}}+\mathcal{O}\left(k h^{2}\right),
$$

and from (4.77) and (4.82), using that $-1<z_{j}<0$,

$$
a_{5, j}^{n}>\frac{k}{h^{2}}\left(\frac{D_{5}}{4 r^{n}}+\frac{h}{2} \kappa\left(\phi \phi_{\omega}\right)^{1-p-q} \frac{\left(u_{1,0}^{n}\right)^{p}\left(u_{3,0}^{n}\right)^{q}}{\sqrt{r^{n}} u_{5,0}^{n}}+\mathcal{O}\left(h^{3}\right)\right) .
$$




\subsection{Numerical analysis: stability and positivity}

Hence, coefficients $a_{5, j}^{n}$ are positive for $-M+1 \leq j \leq-1$.

Now, regarding the concentrations of the chemical species in the uncarbonated zone, the positivity of the coefficients $a_{i, j}^{n}, i \in\{3,6\}$, in equations 4.77)-(4.78) and 4.79 will be probed in analogous way using the value of the difference $r^{n+1}-r^{n}$ that appears in the transmission conditions (4.69).

For $i=3$, using (4.69) and (4.104), it follows that

$$
r^{n+1}-r^{n}=3 D_{3} \frac{k}{h} \frac{\sqrt{r^{n}}}{L-\sqrt{r^{n}}}+2 k \kappa\left(\phi \phi_{\omega}\right)^{1-p-q} \sqrt{r^{n}}\left(u_{1,0}^{n}\right)^{p}\left(u_{3,0}^{n}\right)^{q-1}+\mathcal{O}\left(k h^{2}\right),
$$

and from 4.78) and 4.82, using that $0<z_{j} \leq 1$,

$$
a_{3, j}^{n}>\frac{k}{h^{2}}\left(\frac{D_{3}}{4\left(L-\sqrt{r^{n}}\right)^{2}}-\frac{h}{2} \kappa\left(\phi \phi_{\omega}\right)^{1-p-q} \frac{\left(u_{1,0}^{n}\right)^{p}\left(u_{3,0}^{n}\right)^{q-1}}{\left(L-\sqrt{r^{n}}\right)}+\mathcal{O}\left(h^{3}\right)\right) .
$$

Note that the last expression 4.117), in an analogous way to the equation 4.109, presents a negative term, and positivity of 4.117), for $1 \leq j \leq M$, is guaranteed using the same arguments regarding the boundedness of $u_{1,0}^{n}$ and $u_{3,0}^{n}$, that was proved above for each time level $n$, with the following condition on the spatial step size

$$
h_{3}<\frac{D_{3}}{2\left(L-\sqrt{r^{0}}\right) \kappa\left(\phi \phi_{\omega}\right)^{1-p-q}\left(\tilde{G}_{1}\right)^{p}\left(\tilde{G}_{3}\right)^{q-1}} .
$$

Finally, for $i=6$, taking into account (4.69) and (4.104), it follows that

$$
r^{n+1}-r^{n}=3 D_{6} \frac{k}{h} \frac{\sqrt{r^{n}}}{L-\sqrt{r^{n}}}+\mathcal{O}\left(k h^{2}\right),
$$

and from 4.78) and (4.82, using that $0<z_{j} \leq 1$,

$$
a_{6, j}^{n}>\frac{k}{h^{2}}\left(\frac{D_{6}}{4\left(L-\sqrt{r^{n}}\right)^{2}}+\mathcal{O}\left(h^{3}\right)\right),
$$

and coefficients $a_{6, j}^{n}$ are positive for $1 \leq j \leq M$.

In conclusion, from (4.111) and (4.118), coefficients $a_{i, j}^{n}, 1 \leq i \leq 6, i \neq 4$, are positive under the condition on the spatial step size

$$
h<h_{0}=\min \left\{h_{1}, h_{3}\right\}
$$




\section{6 SPECIES MODEL FOR DAMAGE BY CARBONATION OF REINFORCED CONCRETE STRUCTURES}

Since $0<r^{0}<r^{n}$, the nonnegativity of the coefficients $b_{i, j}^{n}, i \in\{1,2,5\}$, see (4.80), of the explicit scheme 4.77) is guaranteed, independently of the value of $n$, under the following respective conditions between the step sizes $h$ and $k$

$$
k_{1} \leq \frac{h^{2} r^{0}}{2 D_{1}+h^{2} r^{0} P_{1}}, \quad k_{2} \leq \frac{h^{2} r^{0}}{2 D_{2}+h^{2} r^{0} P_{2} Q_{2}}, \quad k_{5} \leq \frac{h^{2} r^{0}}{2 D_{5}}, \quad i \in\{1,2,5\} .
$$

The coefficients related to the uncarbonated zone $b_{i, j}^{n}, i \in\{3,6\}$, see 4.80$\}$, of the explicit scheme (4.78) are non negative, independently of the value of $n$, under the following conditions between $h$ and $k$

$$
k_{3} \leq \frac{h^{2} L^{2}(1-\beta)^{2}}{2 D_{3}+h^{2} L^{2}(1-\beta)^{2} S_{3, \mathrm{diss}}}, \quad k_{6} \leq \frac{h^{2} L^{2}(1-\beta)^{2}}{2 D_{6}}, \quad i \in\{3,6\} .
$$

Then, coefficients $b_{i, j}^{n}, 1 \leq i \leq 6, i \neq 4$, are positive under the condition

$$
k<k_{0}=\min \left\{k_{i}\right\}, \quad 1 \leq i \leq 6, \quad i \neq 4
$$

Consequently, from previous comments and induction argument, the numerical solution at time level $n+1$ is non negative, $u_{i, j}^{n+1} \geq 0, i \neq 4,-M \leq j \leq M$, under conditions (4.121) and (4.124). Now, let us study the boundedness of the numerical solution.

Using (4.101) and 4.104, it holds

$$
\begin{aligned}
& u_{i, 0}^{n}<\frac{2}{3} u_{i,-1}^{n} \leq \tilde{G}_{i}, \quad i \in\{1,2,5\}, \\
& u_{i, 0}^{n}<\frac{2}{3} u_{i, 1}^{n} \leq \tilde{G}_{i}, \quad i \in\{3,6\} .
\end{aligned}
$$

Hence, from (4.77)-(4.78) and 4.79)-4.81), together with assumptions (4.95) and 4.96, one gets

$$
\begin{aligned}
& u_{1, j}^{n+1} \leq\left(a_{1, j}^{n}+b_{1, j}^{n}+c_{1, j}^{n}\right) \tilde{G}_{1}+k P_{1} Q_{1} \tilde{G}_{2}=\left(1-k P_{1}\right) \tilde{G}_{1}+k P_{1} Q_{1} \tilde{G}_{2} \\
& \leq \tilde{G}_{1}, \quad-M+1 \leq j \leq-1, \\
& u_{2, j}^{n+1} \leq\left(a_{2, j}^{n}+b_{2, j}^{n}+c_{2, j}^{n}\right) \tilde{G}_{2}+k P_{2} \tilde{G}_{1}=\left(1-k P_{2} Q_{2}\right) \tilde{G}_{2}+k P_{2} \tilde{G}_{1} \\
& \leq \tilde{G}_{2}, \quad-M+1 \leq j \leq-1,
\end{aligned}
$$




$$
\begin{gathered}
u_{3, j}^{n+1} \leq\left(a_{3, j}^{n}+b_{3, j}^{n}+c_{3, j}^{n}\right) \tilde{G}_{1}+k S_{3, \mathrm{diss}} u_{3, \mathrm{eq}}^{n} \\
=\left(1-k S_{3, \mathrm{diss}}\right) \tilde{G}_{3}+k S_{3, \mathrm{diss}} u_{3, \mathrm{eq}}^{n} \leq \tilde{G}_{3}, \quad 1 \leq j \leq M, \\
u_{5, j}^{n+1} \leq\left(a_{5, j}^{n}+b_{5, j}^{n}+c_{5, j}^{n}\right) \tilde{G}_{5}=\tilde{G}_{5}, \quad-M+1 \leq j \leq-1, \\
u_{6, j}^{n+1} \leq\left(a_{6, j}^{n}+b_{6, j}^{n}+c_{6, j}^{n}\right) \tilde{G}_{6}=\tilde{G}_{6}, \quad 1 \leq j \leq M .
\end{gathered}
$$

From the induction proof for $u_{1,0}^{n}$ and $u_{3,0}^{n}$, and their bounds, we have

$$
0<u_{1,0}^{n}<\frac{2}{3} \tilde{G}_{1}, \quad 0<u_{3,0}^{n}<\frac{2}{3} \tilde{G}_{3} .
$$

From 4.83 and 4.131, taking into account the initial value $u_{4}^{0}=U_{40}$,

$$
u_{4}^{n}>u_{4}^{n-1} ; \quad u_{4}^{n} \leq U_{40}+\kappa T\left(\phi \phi_{\omega}\right)^{1-p-q}\left(\frac{2}{3} \tilde{G}_{1}\right)^{p}\left(\frac{2}{3} \tilde{G}_{3}\right)^{q}, \quad 1 \leq n \leq N .
$$

Using a mathematical induction argument and summarizing, under hypotheses (4.95)-(4.98), the following theorem shows that the numerical solution of problem (4.8)-4.22), obtained from the scheme 4.77)-(4.78) and (4.83), preserves the qualitative properties satisfied by the theoretical solution obtained in Section 3 of [59]:

Theorem 8. With previous notation, under hypotheses (4.95)-(4.98), for small enough values of the step size $h$, verifying (4.111), (4.118) and (4.121), together with the step sizes conditions (4.122)-(4.124), the following conclusions hold true at the mesh points of the numerical domain:

i) Approximate concentrations $u_{i, j}^{n}, i \in\{1,2,5\}$ of the scheme (4.77) in the carbonated zone are positive and uniformly bounded,

$$
0 \leq u_{i, j}^{n} \leq \tilde{G}_{i}, \quad-M \leq j \leq-1, \quad 0 \leq n \leq N
$$

ii) Approximate concentrations $u_{i, j}^{n}, i \in\{3,6\}$ of the scheme (4.78) in the uncarbonated region and uniformly bounded are positive,

$$
0 \leq u_{i, j}^{n} \leq \tilde{G}_{i}, \quad 1 \leq j \leq M, \quad 0 \leq n \leq N
$$




\section{6 SPECIES MODEL FOR DAMAGE BY CARBONATION OF REINFORCED CONCRETE STRUCTURES}

iii) The solution $u_{4}^{n}$ of the scheme (4.83) for the calcium carbonate concentration is positive, increasing and bounded,

$$
u_{4}^{n} \leq U_{40}+\kappa T\left(\phi \phi_{\omega}\right)^{1-p-q}\left(\frac{2}{3} \tilde{G}_{1}\right)^{p}\left(\frac{2}{3} \tilde{G}_{3}\right)^{q}, \quad 0 \leq n \leq N .
$$

iv) Approximate concentrations $u_{i, 0}^{n}, 1 \leq i \leq 6, i \neq 4$ at the carbonation front are positive and uniformly bounded for $0 \leq n \leq N$.

v) The carbonation front is positive and increasing, $0<r^{0}<r^{1}<\ldots<r^{N}$.

As a consequence of the boundedness of the mass concentrations, the stability of the numerical solution is also proved. Let us denote the supremum norm of a vector $x=\left(x_{1}, x_{2}, \ldots, x_{n}\right)^{T}$ in $\mathbb{R}^{n}$ as $\|x\|_{\infty}=\max \left(\left|x_{1}\right|,\left|x_{2}\right|, \ldots,\left|x_{n}\right|\right)$.

According to the notion of stability (1.18) presented in Chapter 1, we will adopt the following definition:

Definition 4. With previous notation, let us denote the vectors of concentrations $u_{i}^{n}=\left[u_{i,-M}^{n}, u_{i,-M+1}^{n}, \ldots, u_{i, 0}^{n}\right]^{T}, i \in\{1,2,5\}$, and $u_{i}^{n}=\left[u_{i, 0}^{n}, u_{i, 1}^{n}, \ldots, u_{i, M}^{n}\right]^{T}$, $i \in\{3,6\}$. We say that the numerical solution $\left\{u_{i, j}^{n}, 1 \leq i \leq 6, i \neq 4, u_{4}^{n}, 0 \leq\right.$ $n \leq N\}$ is $\|\cdot\|_{\infty}$-stable if there exist positive constants $C_{i}, 1 \leq i \leq 6$, independent of $n, k$ and $h$, such that

$$
\left\|u_{i}^{n}\right\|_{\infty} \leq C_{i}, \quad 1 \leq i \leq 6, \quad 0 \leq n \leq N
$$

Thus, scheme (4.77)-4.83) is $\|\cdot\|_{\infty}$-stable under assumptions 4.95)-(4.98), with the step sizes conditions (4.111), (4.118) and (4.121), together with 4.122)(4.124), by taking

$$
C_{i}=\tilde{G}_{i}, \quad 1 \leq i \leq 6, \quad i \neq 4
$$

and

$$
C_{4}=U_{40}+\kappa T\left(\phi \phi_{\omega}\right)^{1-p-q}\left(\frac{2}{3} \tilde{G}_{1}\right)^{p}\left(\frac{2}{3} \tilde{G}_{3}\right)^{q} .
$$

Note that the results of Theorem 8 are conditioned to the step sizes restrictions stated there. The following example illustrates that these conditions can not be removed.

Example 1. Consider the carbonation model (4.8)-(4.22) with parameters listed in the Table 4.1, see [60], with partial reaction orders $p=1.0, q=1.0$ and time 
horizon $T=1$ year, we obtain $h_{0}=0.0041$ and $k_{0}=0.0089$. Taking step sizes with values $h=0.05$ and $k=0.0106$, the positivity condition is broken. Figure 4.1 shows that positivity does not hold. Units in $\mathrm{x}$-axis are taken in $\mathrm{cm}$ and $\mathrm{y}$-axis in $\mathrm{g} \mathrm{cm}^{-3}$.

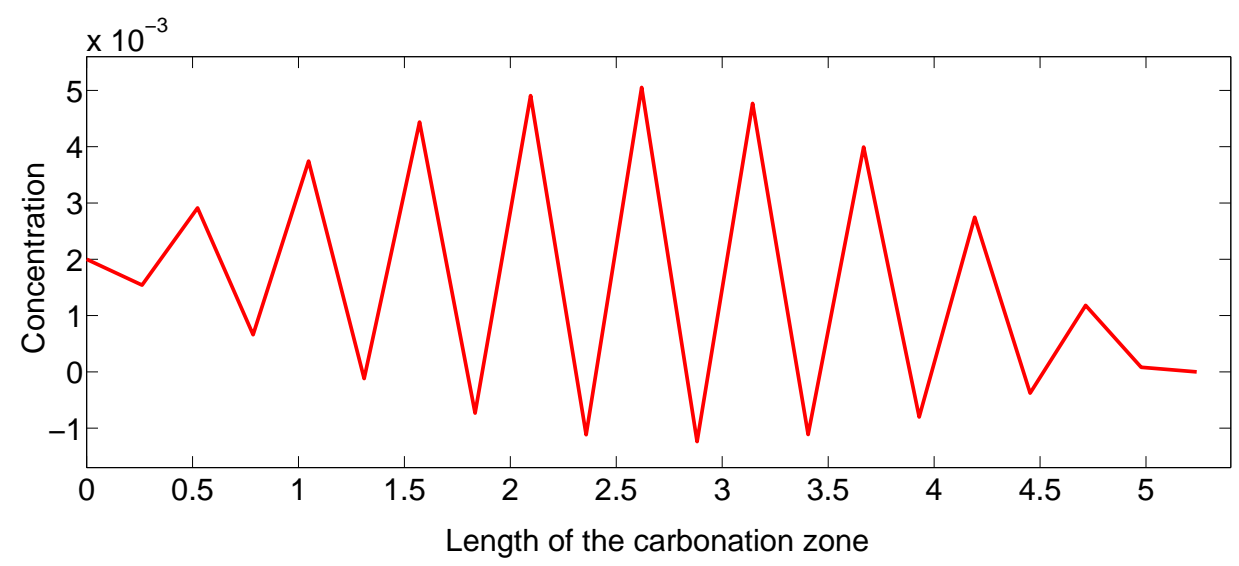

Figure 4.1: Numerical solution $\bar{U}_{1}(x, t)$ of Example 1 for $t=0.95$ years, when positivity condition is broken.

Next example illustrates the positivity and $\|\cdot\|_{\infty}$-stability of the solutions when conditions 4.111, (4.118) and 4.121), together with 4.122)-4.124) are satisfied.

Example 2. With notation and model parameters listed in the Table 4.1, with $p=1.5, q=1.0$ and time horizon $T=10$ years, we get $h_{0}=0.0917$ and $k_{0}=0.0089$. Choosing step sizes $h=0.05$ and $k=0.005$, the positivity and stability of the solutions are guaranteed by Theorem 8 , as Figure 4.2 shows. Units in $\mathrm{x}$-axis are taken in $\mathrm{cm}$ and $\mathrm{y}$-axis in $\mathrm{g} \mathrm{cm}^{-3}$. Furthermore, taking these step size values, the inequality (4.93) is satisfied, since $L=12 \mathrm{~cm}, \rho_{1}=-1.3120$ and the numerical carbonation front $\sqrt{r^{n}}$ together with $\rho_{2, n}$ take the values shown in Figure 4.3. Thus, nonlinear equation (4.71) for $u_{3,0}^{n}$ is solvable and Algorithm 2 can be initiated. Units in x-axis are taken in years and y-axis in cm. Table 4.2 shows CPU time taken in calculations for $\mathrm{h}=0.05$ and several values of $\mathrm{k}$, using MATLAB R2017b on processor Intel(R) Core(TM) i3-3110M CPU $2.40 \mathrm{GHz}$. 


\section{6 SPECIES MODEL FOR DAMAGE BY CARBONATION OF REINFORCED CONCRETE STRUCTURES}
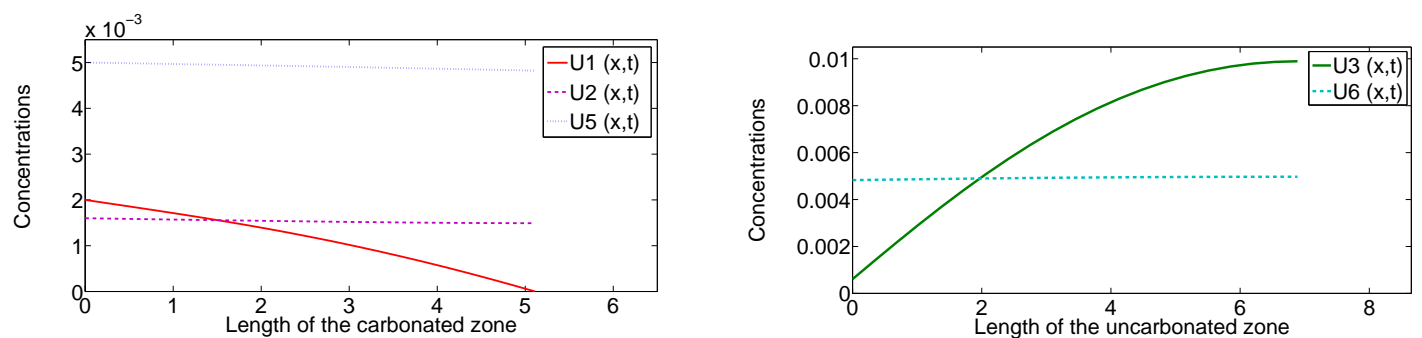

Figure 4.2: Numerical concentrations $\bar{U}_{i}(x, t), i \in\{1,2,5\}$, and $\bar{U}_{i}(x, t), i \in\{3,6\}$, in Example 2 for $t=9$ years, under stability condition.
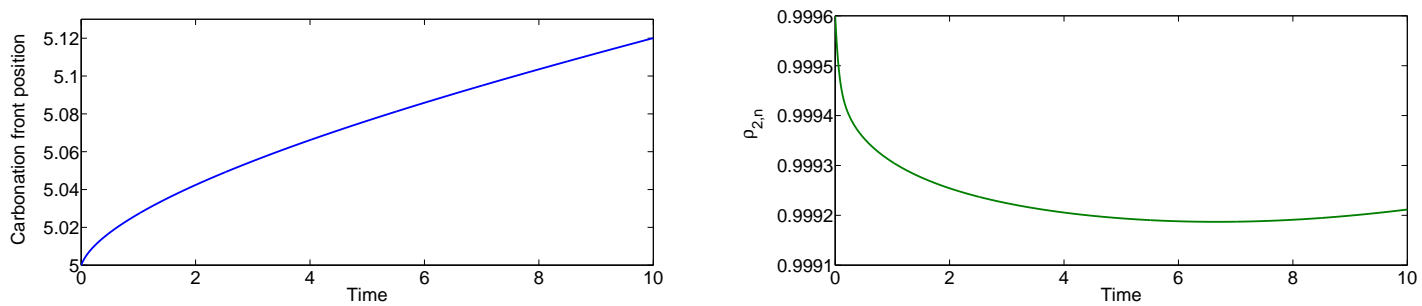

Figure 4.3: Position of the numerical carbonation front $\sqrt{r^{n}}$, and values of $\rho_{2, n}$, in Example 2, as a function of time.

\begin{tabular}{lcccc}
\hline \hline$k$ & 0.008 & 0.006 & 0.004 & 0.002 \\
\hline$\sqrt{r^{N}}(\mathrm{~cm})$ & 5.1200 & 5.1200 & 5.1201 & 5.1201 \\
\hline CPU time (s) & 1.081 & 1.676 & 2.683 & 6.899 \\
\hline \hline
\end{tabular}

Table 4.2: Dependence of process time on the temporal step size.

\subsection{Monotonicity of the numerical solution}

In this section, we present monotone properties of the numerical solution of the scheme (4.77)-(4.78), according to the following definition, see [35]:

Definition 5. Let $F\left(w_{j}^{n}\right)=0$ be a numerical scheme, where index $n$ refers to the time and $j$ to the space, $j \in \mathcal{J}, n \in \mathcal{N}$. We say that the numerical scheme 
$F\left(w_{j}^{n}\right)=0$ is spatial monotone time preserving if, assuming that $w^{n}$ is a spatial monotone sequence at time level $n$, then so is $w^{n+1}$ for all time index $n$.

We will state that the scheme (4.77)-(4.78) preserves the monotone decreasing spatial behaviour of the numerical concentrations $u_{i}^{n}, i \in\{1,2,5\}$, in the carbonated zone, while the concentrations $u_{i}^{n}, i \in\{3,6\}$, in the uncarbonated zone are monotone increasing in space, for all time level $n$. We will prove this property using the induction principle on the temporal index $n$.

Firstly, we state that, under the positivity constraints (4.111), (4.118) and (4.121), together with (4.122)-(4.124), and assuming

$$
u_{i, j+1}^{n} \leq u_{i, j}^{n}, \quad-M+1 \leq j \leq-2, \quad i \in\{1,2,5\},
$$

then, the numerical solution at time level $n+1$ satisfies

$$
u_{i, j+1}^{n+1} \leq u_{i, j}^{n+1}, \quad-M+1 \leq j \leq-2, \quad i \in\{1,2,5\} .
$$

Let us start by considering $i=1$. From (4.77) and (4.139) one gets

$$
\begin{aligned}
& u_{1, j}^{n+1} \geq a_{1, j}^{n} u_{1, j}^{n}+b_{1, j}^{n} u_{1, j}^{n}+c_{1, j}^{n} u_{1, j+1}^{n}+k P_{1} Q_{1} u_{2, j}^{n} \\
& =\left(1-\frac{D_{1} k}{h^{2} r^{n}}-\left(\frac{1+z_{j}}{4 h}\right) \Delta_{1}^{n}-k P_{1}\right) u_{1, j}^{n}+\left(\frac{D_{1} k}{h^{2} r^{n}}+\left(\frac{1+z_{j}}{4 h}\right) \Delta_{1}^{n}\right) u_{1, j+1}^{n} \\
& +k P_{1} Q_{1} u_{2, j}^{n}, \quad-M+1 \leq j \leq-2
\end{aligned}
$$

and

$$
\begin{aligned}
& u_{1, j+1}^{n+1} \leq a_{1, j+1}^{n} u_{1, j}^{n}+b_{1, j+1}^{n} u_{1, j+1}^{n}+c_{1, j+1}^{n} u_{1, j+1}^{n}+k P_{1} Q_{1} u_{2, j+1}^{n} \\
& =\left(\frac{D_{1} k}{h^{2} r^{n}}-\left(\frac{1+z_{j+1}}{4 h}\right) \Delta_{1}^{n}\right) u_{1, j}^{n}+\left(1-\frac{D_{1} k}{h^{2} r^{n}}+\left(\frac{1+z_{j+1}}{4 h}\right) \Delta_{1}^{n}-k P_{1}\right) u_{1, j+1}^{n} \\
& +k P_{1} Q_{1} u_{2, j+1}^{n}, \quad-M+1 \leq j \leq-2
\end{aligned}
$$

Using the positivity of coefficient $b_{1, j}^{n}$ and $\Delta_{1}^{n}$ shown in Theorem 2 from 4.80) for $i=1$, 4.139) and (4.141)-4.142), it follows that

$$
\begin{aligned}
& u_{1, j+1}^{n+1}-u_{1, j}^{n+1} \leq\left(b_{1, j}^{n}+\frac{1}{4} \Delta_{1}^{n}\right)\left(u_{1, j+1}^{n}-u_{1, j}^{n}\right) \\
& +k P_{1} Q_{1}\left(u_{2, j+1}^{n}-u_{2, j}^{n}\right) \leq 0, \quad-M+1 \leq j \leq-2,
\end{aligned}
$$




\section{6 SPECIES MODEL FOR DAMAGE BY CARBONATION OF REINFORCED CONCRETE STRUCTURES}

and then

$$
u_{1, j+1}^{n+1} \leq u_{1, j}^{n+1} .
$$

For the sake of brevity and because of the analogy of the cases $i=2$ and $i=5$, we omit the proofs of the decreasing monotone behaviour of $w_{2}^{n}$, and $w_{5}^{n}$,

Regarding the concentrations in the uncarbonated zone $u_{i, j}^{n}, i \in\{3,6\}$, we will prove that spatial increasing monotonicity requires some additional restrictions on the step sizes discretizations. Thus, under these additional restrictions, and the positivity conditions (4.111), 4.118) and (4.121), together with 4.122)-4.124), if we assume

$$
u_{i, j+1}^{n} \geq u_{i, j}^{n}, \quad 1 \leq j \leq M-1, \quad i \in\{3,6\}
$$

then, the numerical solution at time level $n+1$ satisfies

$$
u_{i, j+1}^{n+1} \geq u_{i, j}^{n+1}, \quad 1 \leq j \leq M-1, \quad i \in\{3,6\}
$$

Let us start by considering the case $i=3$. From (4.78) and (4.145), one gets

$$
\begin{aligned}
& u_{3, j}^{n+1} \leq a_{3, j}^{n} u_{3, j}^{n}+b_{3, j}^{n} u_{3, j}^{n}+c_{3, j}^{n} u_{3, j+1}^{n}+k S_{3, \mathrm{diss}} u_{3, \mathrm{eq}}^{n} \\
& =\left(1-\frac{D_{3} k}{h^{2} \Delta_{3}^{n}}+\left(\frac{z_{j}-1}{4 h}\right) r^{n} \frac{\Delta_{1}^{n} \Delta_{2}^{n}}{\Delta_{3}^{n}}-k S_{3, \mathrm{diss}}\right) u_{3, j}^{n} \\
& +\left(\frac{D_{3} k}{h^{2} \Delta_{3}^{n}}+\left(\frac{1-z_{j}}{4 h}\right) r^{n} \frac{\Delta_{1}^{n} \Delta_{2}^{n}}{\Delta_{3}^{n}}\right) u_{3, j+1}^{n}+k S_{3, \mathrm{diss}} u_{3, \mathrm{eq}}^{n}, \quad 1 \leq j \leq M-1,
\end{aligned}
$$

and

$$
\begin{aligned}
& u_{3, j+1}^{n+1} \geq a_{3, j+1}^{n} u_{3, j}^{n}+b_{3, j+1}^{n} u_{3, j+1}^{n}+c_{3, j+1}^{n} u_{3, j+1}^{n}+k S_{3, \mathrm{diss}} u_{3, \mathrm{eq}}^{n} \\
& =\left(\frac{D_{3} k}{h^{2} \Delta_{3}^{n}}+\left(\frac{z_{j+1}-1}{4 h}\right) r^{n} \frac{\Delta_{1}^{n} \Delta_{2}^{n}}{\Delta_{3}^{n}}\right) u_{3, j}^{n}+k S_{3, \mathrm{diss}} u_{3, \mathrm{eq}}^{n} \\
& +\left(1-\frac{D_{3} k}{h^{2} \Delta_{3}^{n}}+\left(\frac{1-z_{j+1}}{4 h}\right) r^{n} \frac{\Delta_{1}^{n} \Delta_{2}^{n}}{\Delta_{3}^{n}}-k S_{3, \mathrm{diss}}\right) u_{3, j+1}^{n}, \quad 1 \leq j \leq M-1 .
\end{aligned}
$$


Using the positivity of $b_{3, j}^{n}$ and $\Delta_{1}^{n}$ shown in Theorem from f.80 for $i=3$, (4.139) and (4.141)-(4.142), it follows that

$$
u_{3, j+1}^{n+1}-u_{3, j}^{n+1} \geq\left(b_{3, j}^{n}-\frac{r^{n}}{4} \frac{\Delta_{1}^{n} \Delta_{2}^{n}}{\Delta_{3}^{n}}\right)\left(u_{3, j+1}^{n}-u_{3, j}^{n}\right) \geq 0, \quad 1 \leq j \leq M-1 .
$$

Note that the left hand side of expression (4.149) is positive if the bracket coefficient is also positive. From (4.73), (4.82) and 4.110), one gets

$$
\frac{r^{n}}{4} \frac{\Delta_{1}^{n} \Delta_{2}^{n}}{\Delta_{3}^{n}}=\frac{r^{n+1}-r^{n}}{4 \sqrt{r^{n}}\left(L-\sqrt{r^{n}}\right)} \leq \frac{k \alpha \kappa\left(\phi \phi_{\omega}\right)^{1-p-q} \tilde{G}_{1}^{p} \tilde{G}_{3}^{q-1}}{2 L(1-\beta)} .
$$

Thus, from 4.80 for $i=3$, 4.149 and 4.150, the difference $u_{3, j+1}^{n+1}-u_{3, j}^{n+1}$ is non negative under the following requirement linking spatial and temporal step sizes:

$$
k \leq k_{3}^{*}=\frac{2 h^{2} L^{2}(1-\beta)^{2}}{4 D_{3}+2 h^{2} L^{2}(1-\beta)^{2} S_{3, \text { diss }}+h^{2} L(1-\beta) \alpha \kappa\left(\phi \phi_{\omega}\right)^{1-p-q} \tilde{G}_{1}^{p} \tilde{G}_{3}^{q-1}} .
$$

Finally, for the case $i=6$, using similar arguments, it is easy to show that $u_{6}^{n}$, presents a spatial increasing monotone behaviour under the additional condition on the relation between spatial and temporal step sizes:

$$
k \leq k_{6}^{*}=\frac{2 h^{2} L^{2}(1-\beta)^{2}}{4 D_{6}+h^{2} L(1-\beta) \alpha \kappa\left(\phi \phi_{\omega}\right)^{1-p-q} \tilde{G}_{1}^{p} \tilde{G}_{3}^{q-1}} .
$$

Summarizing, the following result can be established:

Theorem 9. With previous notation, under hypotheses (4.95)-(4.98), assuming the positivity conditions (4.121) and (4.124), and the temporal step size conditions 4.151) and (4.152), the numerical scheme (4.77)-(4.83) is spatial monotone preserving in the sense of 5

Consequently, starting with $u_{i}^{0}$ usually spatial monotone decreasing sequences, $i \in\{1,2,5\}$, the numerical solution remains monotone decreasing for all $1 \leq$ $n \leq N$. On the other hand, starting with $u_{i}^{0}$, usual spatially monotone increasing sequences, $i \in\{3,6\}$, the numerical solution remains monotone increasing for all $n$. Next Example 3 illustrates these facts. 


\section{6 SPECIES MODEL FOR DAMAGE BY CARBONATION OF REINFORCED CONCRETE STRUCTURES}

Example 3. With data of Table 4.1, taking reaction orders $p=1.5, q=1$ and time horizon $T=10$ years, with previous notation, we obtain $h_{0}=0.0917$, $k_{0}=0.0089, k_{3}^{*}=0.0059$ and $k_{6}^{*}=0.0056$. Choosing step sizes $h=0.05$ and $k=0.005$ satisfying the monotonicity requirements of Theorem 9 , Figures 4.4 and 4.5 show the monotone behaviour of the numerical solutions of $\bar{U}_{1}(x, t)$ and $\bar{U}_{3}(x, t)$ for several equidistant fixed values of time.

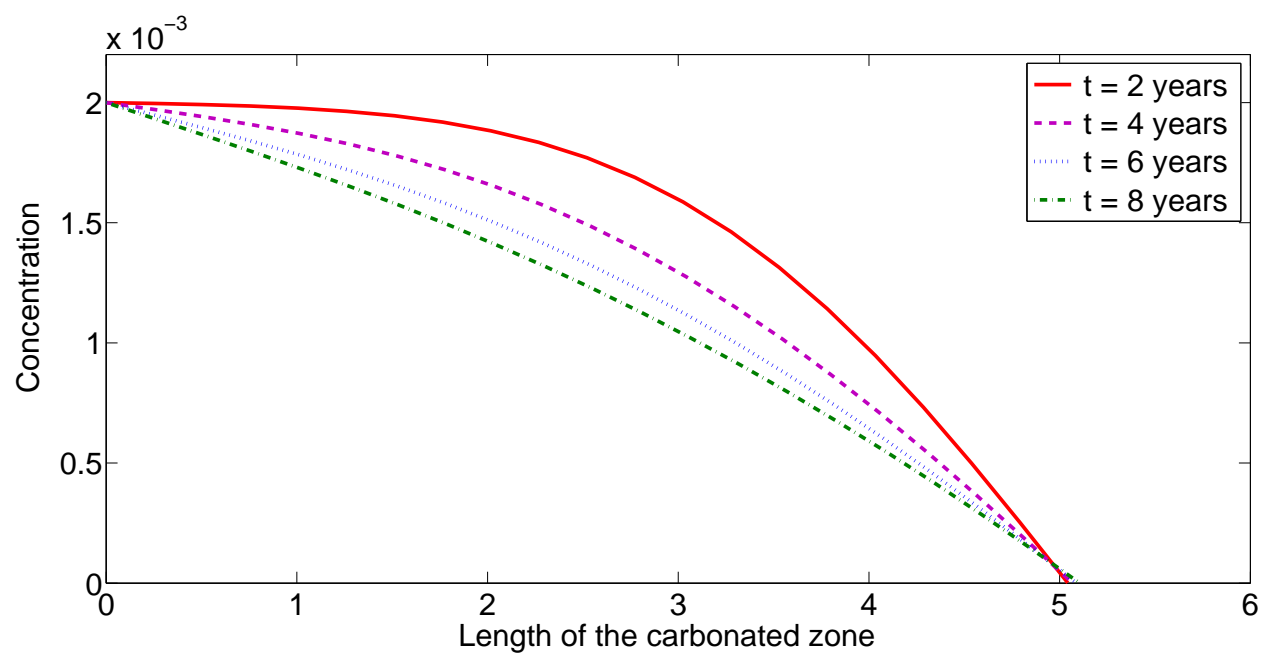

Figure 4.4: Numerical solution $\bar{U}_{1}(x, t)$ of Example 3, for several equidistant times. 


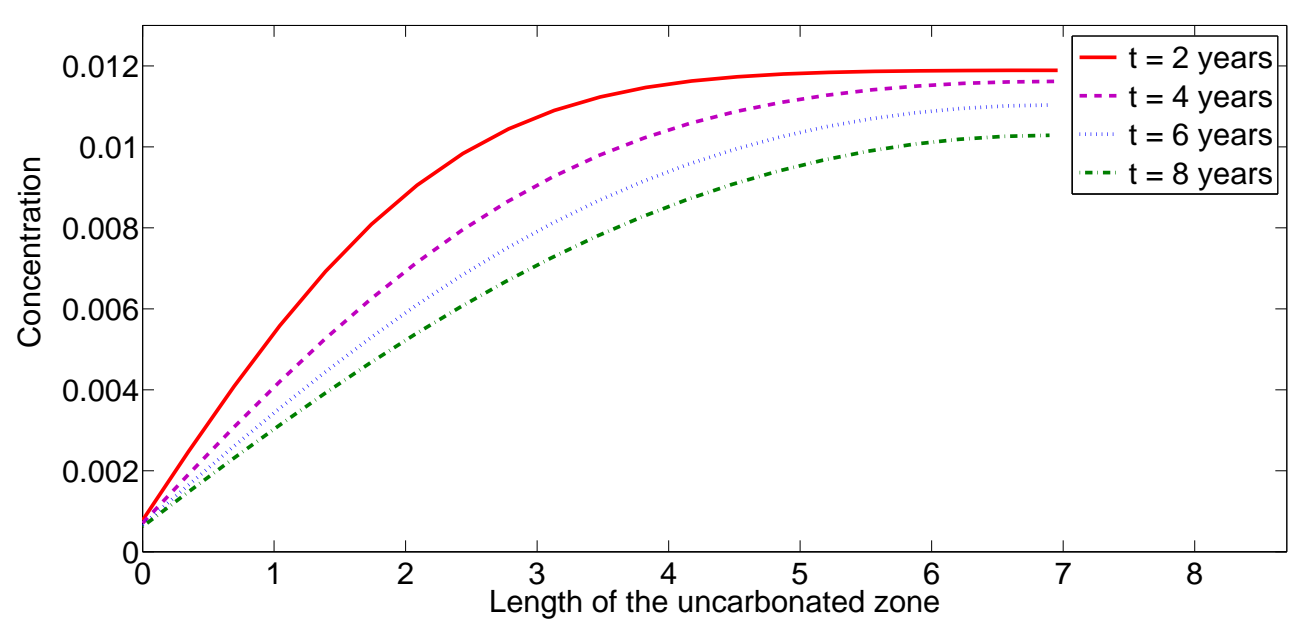

Figure 4.5: Numerical solution $\bar{U}_{3}(x, t)$ of Example 3, for several equidistant times.

\subsection{Conclusions}

From the applications point of view, a theoretical model needs to be checked numerically. In this chapter, we construct reliable numerical solutions of the concrete carbonation model proposed in [59] and [60]. In fact, we show that the proposed numerical solutions are positive and preserve qualitative properties of the theoretical solution such as concentrations boundedness. One the advantages of our approach is that concentrations monotonicity properties suggested in the experiments, although not proved theoretically in [59], are confirmed throughout the behaviour of the numerical solution and illustrated with numerical examples. The numerical analysis includes sufficient conditions on the step sizes discretization, explicitly given in terms of the data in order to satisfy the above properties.

Considering possible future lines of research, it might be convenient to look upon some open questions about the continuos model and its numerical implementation, mainly that regarding the problem of study the carbonation process in sample geometries of 2 or 3 dimensions. Apart from simple cases with any symmetry, radial for instance, that can be treated with the one-dimensional model through a variable change, some attempts have been made to address this challenging issue. 


\title{
4. 6 SPECIES MODEL FOR DAMAGE BY CARBONATION OF REINFORCED CONCRETE STRUCTURES
}

\begin{abstract}
A first result indicates that 1D models applied to the two-dimensional case would underestimate the speed of the deterioration (and overestimate the service life), particularly in singular zones of the element such as convex corners (cf. [69]). The numerical treatment of these problems has been approached via finite elements, but it would be worthwile to apply the finite differente technique used in this thesis to assess its suitability.
\end{abstract}




\begin{tabular}{|c|c|}
\hline Magnitude & Value \\
\hline \multicolumn{2}{|c|}{ Initial concentrations $\left(\mathrm{g} \mathrm{cm}^{-3}\right)$} \\
\hline $\bar{U}_{10}(x), 0<x<S_{0}$ & 0.0020 \\
\hline $\bar{U}_{20}(x), 0<x<S_{0}$ & 0.0016 \\
\hline $\bar{U}_{30}(x), S_{0}<x<L$ & 0.0120 \\
\hline $\bar{U}_{40}$ & 0.0000 \\
\hline $\bar{U}_{50}(x), 0<x<S_{0}$ & 0.0050 \\
\hline $\bar{U}_{60}(x), S_{0}<x<L$ & 0.0050 \\
\hline \multicolumn{2}{|c|}{ Exposed boundary concentrations $\left(\mathrm{g} \mathrm{cm}^{-3}\right)$} \\
\hline$\Lambda_{1}(t)$ & 0.0020 \\
\hline$\Lambda_{2}(t)$ & 0.0016 \\
\hline$\Lambda_{5}(t)$ & 0.0050 \\
\hline \multicolumn{2}{|c|}{ Equilibrium concentration of $\mathrm{Ca}(\mathrm{OH})_{2}\left(\mathrm{~g} \mathrm{~cm}^{-3}\right)$} \\
\hline$U_{3, \mathrm{eq}}$ & 0.0050 \\
\hline \multicolumn{2}{|c|}{ Diffusion constants $\left(\mathrm{cm}^{2}\right.$ day $\left.^{-1}\right)$} \\
\hline$D_{1}$ & 0.62 \\
\hline$D_{2}$ & 3.50 \\
\hline$D_{3}$ & 0.86 \\
\hline$D_{5}$ & 1.00 \\
\hline$D_{6}$ & 1.00 \\
\hline \multicolumn{2}{|l|}{ Model parameters } \\
\hline$\alpha$ & 12500 \\
\hline$\kappa\left(\right.$ year $\left.^{-1}\right)$ & 750 \\
\hline$\phi$ & 0.10 \\
\hline$\phi_{\omega}$ & 0.50 \\
\hline$\phi_{a}$ & 0.50 \\
\hline$P_{1}=P_{2}\left(\right.$ day $\left.^{-1}\right)$ & 0.025 \\
\hline$Q_{1}=Q_{2}$ & 1.250 \\
\hline$S_{3, \text { diss }}\left(\right.$ day $\left.^{-1}\right)$ & 0.0075 \\
\hline \multicolumn{2}{|c|}{ Sample length and bounds of the carbonation depth } \\
\hline$L(\mathrm{~cm})$ & 12.00 \\
\hline$S_{0}(\mathrm{~cm})$ & 5.00 \\
\hline$\beta$ & 0.70 \\
\hline
\end{tabular}

Table 4.1: Data for numerical examples. 



\section{Phase change processes}

As mentioned in Chapter 1, free- or moving-boundary problems are used to model many phenomena arising in science and technology, with a wide range of applications in different disciplines like biophysics, chemistry, astronomy, materials science and ecology [1, 24, 25, 26, 38, 56]. A subset of these kind of models are the so-called Stefan problems linked, for instance, to heat transfer problems with phase changes from the liquid to the solid. Exact analytical solution of these problems is only available in some particular cases stated in a semi-infinite domain, for instance the well known Lamé-Clapeyron-Stefan problem, see [26], Chapter 3. Analytic solutions for two-phase Stefan problems using similarity solutions have been proposed in [12], including source terms in the problem formulation. Apart from the analytical methods, a wide class of semianalytical methods have been proposed to solve more general problems, [16, 18, 40, 65], method of perturbation, [17, 75].

Attending to the treatment of the moving boundary there are two main types of methods, [68]. The front-tracking method is based on the continuous updating of the moving boundary, see Chapter 4 of [26]; inside this method, it is important to mention the variable space grid method [46], the variable time step method [6] and the heat balance integral method [36]. Another approach is to use a fixed domain formulation or front-fixing based on a transformation of the original problem, 


\section{PHASE CHANGE PROCESSES}

for instance the boundary immobilization method, [49], Chapter 5 of [26], [57], isotherm migration method, [20, 47], and enthalpy method, [16, 32, 81]. Both approaches the front-tracking and the front-fixing have been performed using finite difference or finite elements schemes.

The last problem treated in this thesis is related to a specific case of phase change process. The main peculiarity of this problem with respect to those studied in previous chapters lies in the appearance of an additional difficulty, which has to do with the stages of the process when the interface between phases is located at any of the two edges of the material sample. Thus, it follows that two different challenges have to be addressed when the front-fixing transformation is used for two-phase problems due to the appearance of singularity in the transformed partial differential equations (PDEs): Firstly, the correct initialization when the first phase initially has zero thickness, and on the other hand, the treatment of the depletion of the second phase close to the extinction time. The authors of [68] consider the one-phase problem by using front-fixing approaches together with explicit finite difference schemes, overcome the initialization difficulty taking the analytic solution of the semi-infinite problem in this starting stage for the temperature as well as for the evolution of the moving boundary from the starting zero thickness. The correct initialization and depletion of the two-phase Stefan problem has been recently addressed by [57] by using the Keller box implicit difference scheme, see [54].

The numerical modelling proposed in this chapter does not need the analytic solution of the underlying semi-infinite problem but only the hypothesis of the $\sqrt{t}$-law behaviour of the moving boundary in the initialization of the problem. The treatment of the depletion is also addressed throughout a split of the process in three stages: the first one related to the initialization, the second one embracing almost the entire process of solidification and the third stage dealing with the depletion of the liquid phase until extinction. Apart from the numerical analysis, this numerical modelling approach has the potential application of dealing with more complicated problems where the analytic solution of the corresponding semi-infinite problem is not available.

Consider the two-phase model describing the freezing of a liquid in one spatial dimension, [57], occupying initially the region $0 \leq x \leq a$. At the initial time, the 
temperature of the material is uniform, $T_{h}$, higher than the phase-change temperature, written as $T_{f}$. The material is cooled at the point $x=0$ by imposing a constant temperature $T_{c}$, lower than the phase-change temperature, $T_{f}$.

The continuous model with phase formation and depletion is formulated as,

$$
\begin{aligned}
\rho c_{s} \frac{\partial T_{s}}{\partial t} & =\kappa_{s} \frac{\partial^{2} T_{s}}{\partial x^{2}}, \quad 0<x<S(t), \quad t>0, \\
\rho c_{l} \frac{\partial T_{l}}{\partial t} & =\kappa_{l} \frac{\partial^{2} T_{l}}{\partial x^{2}}, \quad S(t)<x<a, \quad t>0 .
\end{aligned}
$$

The boundary conditions are expressed as

$$
\begin{gathered}
T_{s}(0, t)=T_{c}, \quad t>0, \\
T_{s}(S(t), t)=T_{l}(S(t), t)=T_{f}, \quad t>0,
\end{gathered}
$$

and

$$
\frac{\partial T_{l}}{\partial x}(a, t)=0, \quad t>0
$$

The initial conditions are given by

$$
T_{l}(x, 0)=T_{h}, \quad 0 \leq x \leq a, \quad S(0)=0 .
$$

Finally, the so-called Stefan condition, expressing the heat balance on the interface, takes the form

$$
\rho C_{m} \frac{d S}{d t}=\kappa_{s} \frac{\partial T_{s}}{\partial x}-\kappa_{l} \frac{\partial T_{l}}{\partial x}, \quad x=S(t), \quad t>0
$$

Here, $T_{s}$ denotes the solid phase temperature, $\kappa_{s}$ is the thermal conductivity of the solid, $c_{s}$ is the specific heat capacity of the solid phase. Analogously, using the same notation for the liquid phase, $T_{l}$ denotes the liquid phase temperature, $\kappa_{l}$ the thermal conductivity of the liquid and $c_{l}$ the specific heat capacity of the liquid phase. Regarding the mass density of the material, it is assumed to have the same value $\rho$, both in solid and liquid state. The value $C_{m}$ represents the latent heat of fusion. $S(t)$ indicates the location of the solidification front.

Note that as we are considering the freezing problem in accordance with the initial condition (5.6) and the boundary conditions (5.3)-(5.5), the change of phase 
is not reversible, and the right hand side of condition (5.7) has a constant positive sign. Thus,

$$
\rho C_{m} \frac{d S}{d t}>0, \quad t>0
$$

To transform our problem into a dimensionless one, the following nondimensional variables in space and time are used:

$$
y=\frac{x}{a}, \quad \tilde{t}=\frac{\kappa_{l} t}{\rho c_{l} a^{2}}, \quad \tilde{S}(\tilde{t})=\frac{S(t)}{a} .
$$

Consider an arbitrarily small value $S_{0}, 0<S_{0}<a$, a relative quantity $R_{0}=$ $S_{0} / a$ and let us introduce the new temporal variable and the transformed solidification front, respectively

$$
\tau=\tilde{t} / R_{0}^{2}, \quad \sigma(\tau)=R(\tau) / R_{0}, \quad R(\tau)=\tilde{S}(\tilde{t}) .
$$

Also, the unknowns for the temperatures in the solid and liquid phases, respectively, are expressed as

$$
\hat{U}_{s}=\frac{T_{s}-T_{f}}{T_{f}-T_{c}}, \quad \hat{U}_{l}=\frac{T_{l}-T_{f}}{T_{h}-T_{f}} .
$$

After these changes, and simplifying the notation by using the parameters

$$
\kappa=\frac{\kappa_{l} c_{s}}{\kappa_{s} c_{l}}, \quad K=\frac{\kappa_{s}\left(T_{f}-T_{c}\right)}{\kappa_{l}\left(T_{h}-T_{f}\right)}, \quad \beta=\frac{C_{m}}{c_{l}\left(T_{h}-T_{f}\right)},
$$

the problem (5.1)-(5.7) becomes

$$
\begin{gathered}
\frac{\partial \hat{U}_{s}}{\partial \tau}=\frac{R_{0}^{2}}{\kappa} \frac{\partial^{2} \hat{U}_{s}}{\partial y^{2}}, \quad 0<y<R_{0} \sigma(\tau), \quad \tau>0 \\
\frac{\partial \hat{U}_{l}}{\partial \tau}=R_{0}^{2} \frac{\partial^{2} \hat{U}_{l}}{\partial y^{2}}, \quad R_{0} \sigma(\tau)<y<1, \quad \tau>0
\end{gathered}
$$

subject to the boundary conditions

$$
\begin{gathered}
\hat{U}_{s}(0, \tau)=-1, \tau>0 \\
\hat{U}_{s}\left(R_{0} \sigma(\tau), \tau\right)=\hat{U}_{l}\left(R_{0} \sigma(\tau), \tau\right)=0, \quad \tau>0
\end{gathered}
$$

and

$$
\frac{\partial \hat{U}_{l}}{\partial y}(1, \tau)=0, \quad \tau>0
$$


and the new initial conditions

$$
\hat{U}_{l}(y, 0)=1, \quad 0 \leq y \leq 1, \quad \sigma(0)=0 .
$$

Finally, the Stefan condition is written as

$$
\frac{d \sigma}{d \tau}=\frac{K R_{0}}{\beta} \frac{\partial \hat{U}_{s}}{\partial y}-\frac{R_{0}}{\beta} \frac{\partial \hat{U}_{l}}{\partial y}, \quad y=R_{0} \sigma(\tau), \quad \tau>0
$$

The main Section 5.1 begins with the front-fixing transformation of the moving boundary problem. Then, the numerical modelling is developed by splitting the solidification process in three stages: initialization, progress stage and depletion. In both stages, discretization, numerical analysis, including the study of qualitative properties of the numerical solution, the stability and simulation are performed. Illustrative numerical experiments are also included.

\subsection{Front-fixing transformation and numerical mod- elling}

The correct inicialization of the solidification front requires that $S(0)=0$, i.e. $\sigma(0)=0$, see [55], where

$$
\sigma(\tau)=R(\tau) / R_{0}
$$

Let us consider the front-fixing transformation ([26, 49])

$$
z(y, \tau)= \begin{cases}\frac{y-\sigma(\tau) R_{0}}{\sigma(\tau) R_{0}}, & 0 \leq y \leq \sigma(\tau) R_{0}, \quad \tau \geq 0, \\ \frac{y-\sigma(\tau) R_{0}}{1-\sigma(\tau) R_{0}}, & \sigma(\tau) R_{0} \leq y \leq 1, \quad \tau \geq 0 .\end{cases}
$$

As a result of trasform (5.21), the moving-boundary problem (5.13)-(5.19) turns into another one that is posed in a fixed immobilized domain, $-1 \leq z \leq 1, \tau \geq 0$, that causes difficulties in the initialization and in the depletion. The difficulty about $t=0$ can be overcome without considering the analytic solution of the corresponding semi-infinite two-phase Lamé-Clapeyron-Stefan problem, [26, 77], but only assuming that the solidification front in the nondimensional problem follows the 


\section{PHASE CHANGE PROCESSES}

behaviour $C \sqrt{\tau}$ in a small neighbourhood close to $\tau=0$, where $C$ is obtained throughout numerical modelling specified later. Another important issue is the numerical treatment of the problem close to the extinction time $\tau_{e}$, [57, 77], without using the solution of the semi-infinite problem. In fact, in agreement with the comment by the authors of [57], p. 270, we neglect the liquid phase starting from a time $\tau_{1}<\tau_{e}$. The selection of this critical time $\tau_{1}$ is performed in order to guarantee the numerical stability of the solution.

Thus, the numerical modelling of the problem is developed in three stages. The first stage corresponds to the correct initialization of the solidification process, that is performed using an implicit scheme for both purposes, the estimation of $C$ as well as obtaining the temperature of the solid and the liquid phases until a time $\tau_{0}$ such that the solidification front has reached the relative value $R_{0}$.

Then, the second stage corresponds to the evolution of the two-phase problem until we reach time $\tau_{1}$. Note that in this second stage we need to compute both the temperature of solid and liquid phases, as well as the solidification front. This computation is performed using an explicit method that, apart from being quick and no requiring initialization guess, as it occurs with the implicit methods, it will be particularly convenient to study numerical properties of the numerical solution.

Finally, in the last stage we neglect the liquid phase and we study the behaviour of the solidification front and the temperature of the solid region until the extinction time, continuing with the explicit scheme.

Let us choose an arbitrarily small value $S_{0}>0$ and denote $t_{0}>0$ the time such that $S\left(t_{0}\right)=S_{0}$, following the $\sqrt{t}$-law. Taking $\tau_{0}$ the corresponding transformed time throughout 5.10 ) one gets,

$$
\sigma\left(\tau_{0}\right)=\sigma_{0}=\frac{R\left(\tau_{0}\right)}{R_{0}}=\frac{a \tilde{S}\left(\tilde{t}_{0}\right)}{S_{0}}=1 .
$$

Therefore, we assume that the solidification front follows a behaviour $\sigma(\tau)=$ $C \sqrt{\tau}$ in a short first stage $0 \leq \tau \leq \tau_{0}$, dependent on the initial prefixed value $S_{0}$. From (5.9)-(5.20), one gets

$$
\sigma(\tau)=C \sqrt{\tau}, \quad 0 \leq \tau \leq \tau_{0}=\frac{\tilde{t}_{0}}{R_{0}^{2}}=\frac{1}{C^{2}},
$$


and the value $C$ is obtained numerically as it will be explained later. Note that, during this stage, $0 \leq \sigma(\tau) \leq 1$. Hereinafter the tilde in $\tilde{t}$ is dropped.

Hence, from the above variable change (5.20), transformation (5.21) and the relationships

$$
\hat{U}_{s}(y, \tau)=U_{s}(z, \tau), \quad \hat{U}_{l}(y, \tau)=U_{l}(z, \tau),
$$

problem (5.13)-(5.19) takes the form

$$
\begin{gathered}
\frac{\partial U_{s}}{\partial \tau}=\frac{\sigma^{\prime}(\tau)(1+z)}{\sigma(\tau)} \frac{\partial U_{s}}{\partial z}+\frac{1}{\kappa \sigma^{2}(\tau)} \frac{\partial^{2} U_{s}}{\partial z^{2}}, \quad-1<z<0, \quad \tau>0 \\
\frac{\partial U_{l}}{\partial \tau}=\frac{\sigma^{\prime}(\tau) R_{0}(1-z)}{1-\sigma(\tau) R_{0}} \frac{\partial U_{l}}{\partial z}+\frac{R_{0}^{2}}{\left(1-\sigma(\tau) R_{0}\right)^{2}} \frac{\partial^{2} U_{l}}{\partial z^{2}}, \quad 0<z<1, \quad \tau>0
\end{gathered}
$$

satisfying the boundary conditions

$$
\begin{gathered}
U_{s}(-1, \tau)=-1, \quad \tau>0, \\
U_{s}(0, \tau)=U_{l}(0, \tau)=0, \quad \tau>0,
\end{gathered}
$$

and

$$
\frac{\partial U_{l}}{\partial z}(1, \tau)=0, \quad \tau>0
$$

together with the initial conditions

$$
U_{s}(z, 0)=-1, \quad-1 \leq z \leq 0, \quad U_{l}(z, 0)=1, \quad 0 \leq z \leq 1, \quad \sigma(0)=0
$$

and the Stefan condition at the solidification front

$$
\frac{d \sigma}{d \tau}=\frac{K}{\beta \sigma(\tau)} \frac{\partial U_{s}}{\partial z}-\frac{R_{0}}{\beta\left(1-\sigma(\tau) R_{0}\right)} \frac{\partial U_{l}}{\partial z}, \quad z=0, \quad \tau>0
$$

\subsubsection{First stage numerical modelling}

In the first stage, $0 \leq \tau \leq \tau_{0}$, using the $\sqrt{\tau}$-law 5.23 for the dynamics of the solidification front, problem 5.25)-(5.31) becomes

$$
\frac{\partial U_{s}}{\partial \tau}=\frac{1+z}{2 \tau} \frac{\partial U_{s}}{\partial z}+\frac{1}{\kappa C^{2} \tau} \frac{\partial^{2} U_{s}}{\partial z^{2}}, \quad-1<z<0, \quad 0<\tau<\tau_{0}
$$


$\frac{\partial U_{l}}{\partial \tau}=\frac{C R_{0}(1-z)}{2 \sqrt{\tau}\left(1-C R_{0} \sqrt{\tau}\right)} \frac{\partial U_{l}}{\partial z}+\frac{R_{0}^{2}}{\left(1-C R_{0} \sqrt{\tau}\right)^{2}} \frac{\partial^{2} U_{l}}{\partial z^{2}}, \quad 0<z<1, \quad 0<\tau<\tau_{0}$,

together with boundary and initial conditions (5.27)-(5.29) and (5.30), respectively, and the Stefan condition with $\sqrt{\tau}$-law

$$
\frac{C}{2 \sqrt{\tau}}=\frac{K}{\beta C \sqrt{\tau}} \frac{\partial U_{s}}{\partial z}+\frac{R_{0}}{\beta\left(1-C R_{0} \sqrt{\tau}\right)} \frac{\partial U_{l}}{\partial z}, \quad z=0, \quad 0<\tau<\tau_{0},
$$

that is going to be used to estimate numerically the value of $C$.

In order to circumvent the singularity of equations (5.32)-(5.33) at $\tau=0$, we use a fully implicit scheme, backward in time and centred in space. The numerical domain is partitioned by the grid

$$
\Omega=\left\{\left(z_{j}, \tau^{n}\right), \quad-M \leq j \leq M, \quad 0 \leq n \leq n_{0}\right\},
$$

with $h=\Delta z=1 / M ; \Delta \tau=\tau_{0} / n_{0}$, and $z_{j}=j h, \tau^{n}=n \Delta \tau$, where $M$ and $n_{0}$ are positive integers. By using the notation presented in Section 1.3, numerical approximations of the unknowns are written as: $u_{s, j}^{n} \approx U_{s}\left(z_{j}, \tau^{n}\right), u_{l, j}^{n} \approx U_{l}\left(z_{j}, \tau^{n}\right)$, $\sigma^{n} \approx \sigma\left(\tau^{n}\right)$.

To preserve the second order accuracy at the solidification front $z=0$, we take left and right side approximations with three points, for the solid and liquid phase, respectively:

$$
\frac{3 u_{s, 0}^{n}-4 u_{s,-1}^{n}+u_{s,-2}^{n}}{2 h} \approx \frac{\partial U_{s}}{\partial z}\left(0, \tau^{n}\right), \frac{-3 u_{l, 0}^{n}+4 u_{l, 1}^{n}-u_{l, 2}^{n}}{2 h} \approx \frac{\partial U_{l}}{\partial z}\left(0, \tau^{n}\right)
$$

Boundary conditions (5.27)-(5.29) are discretized as

$$
u_{s,-M}^{n}=-1, \quad u_{s, 0}^{n}=u_{l, 0}^{n}=0, \quad \frac{u_{l, M-1}^{n}-u_{l, M+1}^{n}}{2 h}=0, \quad 0 \leq n \leq n_{0},
$$

where $z_{M+1}=(M+1) h$ is an artificial mesh point and the value $u_{l, M+1}^{n}$ is eliminated under the standard assumption that the equation (5.33) is also satisfied at the boundary $z_{M}=1$, [72] .

From the above comments, one gets a tridiagonal algebraic system

$$
\left\{\begin{array}{l}
A^{n+1} U_{s}^{n+1}=U_{s}^{n}, \\
B^{n+1} U_{l}^{n+1}=U_{l}^{n},
\end{array}\right.
$$




\subsection{Front-fixing transformation and numerical modelling}

where $U_{s}^{n}=\left[u_{s,-M}^{n}, u_{s,-M+1}^{n}, \ldots, u_{s, 0}^{n}\right]^{T}, U_{l}^{n}=\left[u_{l, 0}^{n}, u_{l, 1}^{n}, \ldots, u_{l, M}^{n}\right]^{T}$, and

$$
A^{n}=A^{n}(C)=\left(A_{i, j}^{n}\right)_{-M \leq i, j \leq 0}, \quad B^{n}=B^{n}(C)=\left(B_{i, j}^{n}\right)_{0 \leq i, j \leq M}
$$

are tridiagonal matrices of size $(M+1) \times(M+1)$, whose nonzero entries are

$$
\begin{aligned}
& A_{-M,-M}^{n}=A_{0,0}^{n}=1, \\
& A_{j, j-1}^{n}=-\left(a^{n}-c_{j}^{n}\right) ; \quad A_{j, j}^{n}=1+2 a^{n} ; \quad A_{j, j+1}^{n}=-\left(a^{n}+c_{j}^{n}\right), \\
& -M+1 \leq j \leq-1, \\
& B_{0,0}^{n}=1 ; \quad B_{M, M-1}=-2 b^{n}, \quad B_{M, M}=1+2 b^{n}, \\
& B_{j, j-1}^{n}=-\left(b^{n}-d_{j}^{n}\right) ; \quad B_{j, j}^{n}=1+2 b^{n} ; \quad B_{j, j+1}^{n}=-\left(b^{n}+d_{j}^{n}\right), \\
& 1 \leq j \leq M-1 .
\end{aligned}
$$

Coefficients appearing in (5.40)-(5.41), depending on the step sizes $h$ and $\Delta \tau$, have the form:

$$
\begin{aligned}
& a^{n}=\frac{1}{\kappa C^{2} n h^{2}}, \quad c_{j}^{n}=\frac{z_{j}+1}{4 n h} \\
& b^{n}=\frac{R_{0}^{2} \Delta \tau}{h^{2}\left(1-C R_{0} \sqrt{n \Delta \tau}\right)^{2}}, \quad d_{j}^{n}=\frac{C R_{0} \sqrt{\Delta \tau}\left(1-z_{j}\right)}{4 \sqrt{n} h\left(1-C R_{0} \sqrt{n \Delta \tau}\right)} .
\end{aligned}
$$

$A^{n+1}$ and $B^{n+1}$ of 5.38 have all the row sums positive from 5.40)-5.41. Note that if

$$
\mathrm{C} 1: a^{n+1}-c_{j}^{n+1} \geq 0, \quad \mathrm{C} 2: b^{n+1}-d_{j}^{n+1} \geq 0, \quad 0 \leq n \leq n_{0}-1,
$$

respectively, matrices $A^{n+1}$ and $B^{n+1}$ are Z-matrices, i.e., they have all entries nonpositive out of the main diagonal. Then, under conditions $\mathrm{C} 1$ and $\mathrm{C} 2$, matrices $A^{n+1}$ and $B^{n+1}$ are nonsingular M-matrices and their inverses $\left(A^{n+1}\right)^{-1}$ and $\left(B^{n+1}\right)^{-1}$ are nonnegative, [62] and [7], Chapter 6. This property guarantees that the sign of the vector solution of system (5.38) is preserved.

From (5.42) it is easy to check that conditions (5.43) are verified for all $j$ and $n$ if step sizes satisfy the condition

$$
h \leq \min \left\{h_{1}, h_{2}\right\}, \quad h_{1}=\frac{4}{\kappa C^{2}}, \quad h_{2}=\frac{4 R_{0} \sqrt{\Delta \tau}}{C} .
$$

Apart from the sign stability, the implicit scheme 5.38 guarantees the $\|\cdot\|_{\infty^{-}}$ stability of the solution, in the sense that $\left\|U_{s}^{n}\right\|_{\infty}$ and $\left\|U_{l}^{n}\right\|_{\infty}$ remain bounded for 
all $n$. In fact, matrices $A^{n+1}$ and $B^{n+1}$ have the property that all their row sums are equal to one. It is easy to check that then, their positive inverses also satisfy the same property and $\left\|\left(A^{n}\right)^{-1}\right\|_{\infty}=\left\|\left(B^{n}\right)^{-1}\right\|_{\infty}=1$. In fact,

$$
\left\|U_{s}^{n+1}\right\|_{\infty}=\left\|\left(A^{n+1}\right)^{-1} U_{s}^{n}\right\|_{\infty} \leq\left\|\left(A^{n+1}\right)^{-1}\right\|_{\infty}\left\|U_{s}^{n}\right\|_{\infty} .
$$

Then,

$$
\left\|U_{s}^{n}\right\|_{\infty} \leq\left\|U_{s}^{0}\right\|_{\infty}=1, \quad 0 \leq n \leq n_{0}
$$

and analogously,

$$
\left\|U_{l}^{n}\right\|_{\infty} \leq\left\|U_{l}^{0}\right\|_{\infty}=1, \quad 0 \leq n \leq n_{0} .
$$

Furthermore, from (5.46)-(5.47) and taking into account that $U_{s}^{n} \leq 0, U_{l}^{n} \geq 0$, one gets

$$
-1 \leq u_{s, j}^{n} \leq 0, \quad-M \leq j \leq 0 ; \quad 0 \leq u_{l, j}^{n} \leq 1, \quad 0 \leq j \leq M
$$

for all time steps $0 \leq n \leq n_{0}$.

This means that the numerical solution preserves the property that the temperature in the solid phase is greater than the boundary value, and for the liquid phase, the temperature is below the initial temperature at the right boundary.

In order to estimate the value of the constant $C$ related to the $\sqrt{\tau}$-law, i.e $\sigma(\tau)=$ $C \sqrt{\tau}$, we take the first time step of the system 5.38 together with the linking discretization of Stefan condition (5.34), building a system of $2 M+3$ nonlinear algebraic equations,

$$
\left\{\begin{array}{l}
A^{1} U_{s}^{1}=U_{s}^{0}, \\
B^{1} U_{l}^{1}=U_{l}^{0}, \\
\frac{C}{2}=\frac{K}{\beta C} \Delta u_{s, 0}^{1}+\frac{R_{0} \sqrt{\Delta \tau}}{\beta\left(1-C R_{0} \sqrt{\Delta \tau}\right)} \Delta u_{l, 0}^{1}
\end{array}\right.
$$

where

$$
\Delta u_{s, 0}^{1}=\frac{3 u_{s, 0}^{1}-4 u_{s,-1}^{1}+u_{s,-2}^{1}}{2 h}, \Delta u_{l, 0}^{1}=\frac{-3 u_{l, 0}^{1}+4 u_{l, 1}^{1}-u_{l, 2}^{1}}{2 h},
$$

on the $2 M+3$ unknowns

$$
\left\{\left\{u_{s, j}^{1},-M \leq j \leq 0\right\},\left\{u_{l, j}^{1}, 0 \leq j \leq M\right\}, C\right\} .
$$




\subsection{Front-fixing transformation and numerical modelling}

Solving the system (5.49)-(5.50) with the MATLAB optimization algorithm Fsolve, one gets the temperature at the mesh points in the first time level $n=1$ as well as the value of $C$. It is clear that the value of $C$ depends on the parameters $h$, $\Delta \tau$ for a fixed chosen solidified fraction $R_{0}$ of the material at the end of the first stage. In the next table, we show that for different choices of the step sizes discretizations verifying the stability condition (5.43), one gets values of the constant $C$ close to the theoretical value of the semi-infinite two-phase Lamé-ClapeyronStefan problem, see [26], equation (3.11), p. 102.

\begin{tabular}{llllll}
\hline \hline$h$ & 0.0050 & 0.0040 & 0.0030 & 0.0020 & 0.0010 \\
$\Delta \tau$ & 0.0480 & 0.0325 & 0.0200 & 0.0100 & 0.0026 \\
\hline$C$ & 0.7762 & 0.7728 & 0.7684 & 0.7616 & 0.7596 \\
\hline \hline
\end{tabular}

Table 5.1: Dependence of $C$ on the step sizes when the parameters of the model are $\kappa=K=\beta=1$.

Next example illustrates the temperature profile in both phases solid and liquid, obtained by the numerical scheme $(5.38)$ at the end of the fist stage.

Example 1 Numerical solution of system (5.38) for the last time step $n_{0}$ of this first stage such that $\tau_{0}=n_{0} \Delta \tau$, is shown in Figure 5.1. The parameters are $\kappa=K=\beta=1 ; \Delta \tau=0.01, h=0.002, R_{0}=0.03$ and the value $C$, previously obtained, is $C=0.7616$. Note that $R_{0}=0.03$ means that $3 \%$ of the material has solidified. From (5.23), $\tau_{0}=1.72404$. 


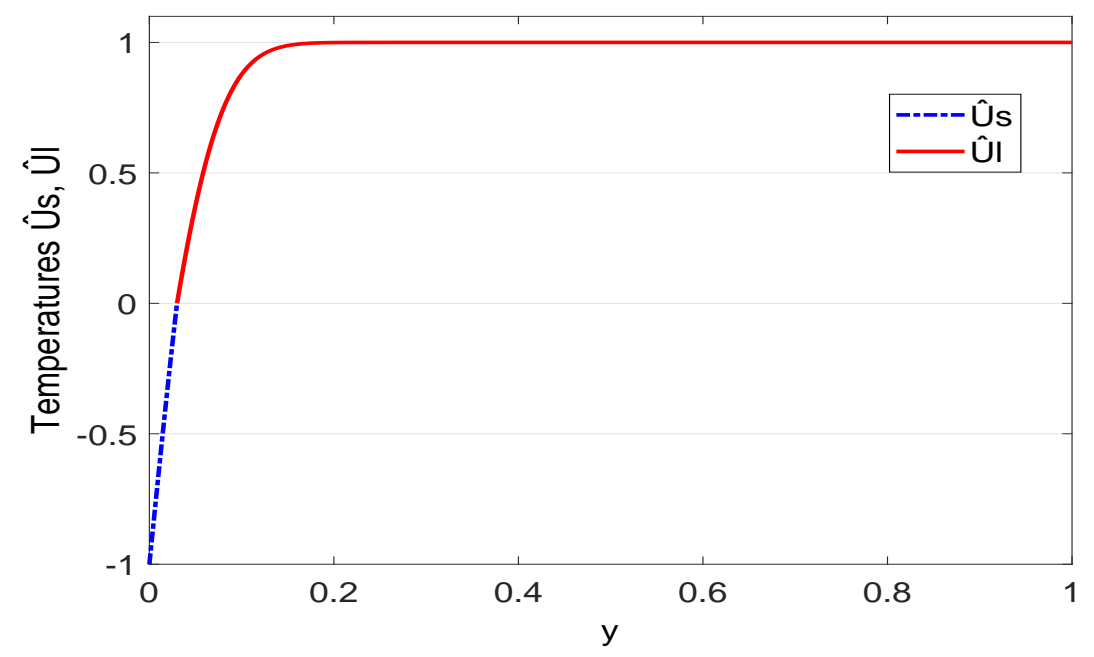

Figure 5.1: Numerical solutions $\hat{U}_{s}(y, \tau), \hat{U}_{l}(y, \tau)$ at the end of the first stage.

\subsubsection{Second stage numerical modelling}

Once the initialization difficulties have been overcome, we address the second stage, where an explicit scheme is used for the numerical solution of equations (5.25)- 5.26) with the Stefan condition 5.31). For this stage, let us take a time step $k$ and the discretized temporal variable $\tau^{n}=\tau_{0}+\left(n-n_{0}\right) k, n_{0} \leq n \leq n_{1}$, where $n_{1}$ is the final time level to be determined by the stability requirements. Note that here, the discretized initial conditions coincide with the final ones of the first stage, while the boundary conditions remain unaltered, 5.37). For the spatial step $h$ and the time step $k$, the explicit scheme for the interior points takes the form

$$
u_{m, j}^{n+1}=a_{m, j}^{n} u_{m, j-1}^{n}+b_{m, j}^{n} u_{m, j}^{n}+c_{m, j}^{n} u_{m, j+1}^{n}, \quad m=s, l, \quad n_{0} \leq n \leq n_{1}-1
$$


where

$$
\begin{aligned}
& a_{s, j}^{n}=\alpha^{n}-\left(1+z_{j}\right) \beta^{n}, \quad b_{s, j}^{n}=1-2 \alpha^{n}, \quad c_{s, j}^{n}=\alpha^{n}+\left(1+z_{j}\right) \beta^{n}, \\
& \alpha^{n}=\frac{k}{h^{2} \kappa\left(\sigma^{n}\right)^{2}}, \quad \beta^{n}=\frac{\sigma^{n+1}-\sigma^{n}}{2 h \sigma^{n}} ; \\
& a_{l, j}^{n}=\gamma^{n}-\left(1-z_{j}\right) \delta^{n}, \quad b_{l, j}^{n}=1-2 \gamma^{n} ; \quad c_{l, j}^{n}=\gamma^{n}+\left(1-z_{j}\right) \delta^{n}, \\
& \gamma^{n}=\frac{k}{h^{2}\left(\rho_{0}-\sigma^{n}\right)^{2}}, \quad \delta^{n}=\frac{\sigma^{n+1}-\sigma^{n}}{2 h\left(\rho_{0}-\sigma^{n}\right)}
\end{aligned}
$$

and

$$
\rho_{0}=\frac{1}{R_{0}}
$$

In the above notation, $m=s$ or $m=l$, where $s$ holds for the material in the solid phase and $l$ for the liquid phase. Note that for the solid phase $-M+1 \leq$ $j \leq-1$, while for the liquid phase $1 \leq j \leq M$, involving the value $u_{l, M+1}^{n}$ at the fictitious mesh point $z_{M+1}$. Finally, the discretization of the Stefan-like condition (5.31) for obtaining the evolution of the solidification front takes the form

$$
\frac{\sigma^{n+1}-\sigma^{n}}{k}=\frac{K}{\beta \sigma^{n}} \Delta u_{s, 0}^{n}-\frac{1}{\beta\left(\rho_{0}-\sigma^{n}\right)} \Delta u_{l, 0}^{n}, \quad n_{0} \leq n \leq n_{1}-1,
$$

where

$$
\Delta u_{s, 0}^{n}=\frac{3 u_{s, 0}^{n}-4 u_{s,-1}^{n}+u_{s,-2}^{n}}{2 h}, \Delta u_{l, 0}^{n}=\frac{-3 u_{l, 0}^{n}+4 u_{l, 1}^{n}-u_{l, 2}^{n}}{2 h},
$$

are the second order approximations of the involved one-sided partial derivatives in 5.31).

Now we study sufficient conditions for the positivity of coefficients in 5.52, because this fact guarantees the preservation of the sign of the solutions (negative for the solid phase and positive for the liquid one). From (5.52)-(5.53), the increasing behaviour of the solidification front and 5.22, the coefficient $b_{s, j}^{n}$ becomes positive if

$$
k<\frac{\kappa h^{2}}{2} .
$$

Using (5.53), Stefan condition 5.55) and the fact that both expressions $h \Delta u_{l, 0}^{n}$ and $h \Delta u_{l, 0}^{n}$ are $\mathcal{O}(h)$, it holds

$$
\frac{\beta^{n}}{\alpha^{n}}=\frac{\kappa h \sigma^{n}\left(\sigma^{n+1}-\sigma^{n}\right)}{2 k}=\frac{\kappa h}{2 \beta}\left(K \Delta u_{s, 0}^{n}-\frac{\sigma^{n}}{\rho_{0}-\sigma^{n}} \Delta u_{l, 0}^{n}\right)=\mathcal{O}(h) .
$$


Thus, $\beta^{n}<\alpha^{n}$ for small enough values of $h$, and from $0<1+z_{j}<1$, one gets the positivity of $a_{s, j}^{n}$, while $c_{s, j}^{n}>0$ unconditionally. In an analogous way, the positivity of coefficients $a_{l, j}^{n}$ and $c_{l, j}^{n}$ in the liquid phase scheme can be proved. Positivity of $b_{l, j}^{n}$ holds under the step sizes condition

$$
k<\frac{h^{2}\left(\rho_{0}-\sigma^{n}\right)^{2}}{2}, \quad n_{0} \leq n \leq n_{1} .
$$

Note that 5.57) guarantees the inequality 5.59) when $\kappa<\left(\rho_{0}-\sigma^{n}\right)^{2}$. This means that the solidification front must satisfy $\sigma^{n}<\rho_{0}-\sqrt{\kappa}$ and hence the integer $n_{1}$ defining the final time level of the second stage is

$$
n_{1}=\max \left\{n: \sigma^{n}<\rho_{0}-\sqrt{\kappa}, ; n \geq n_{0}\right\} .
$$

Note that the final of the second stage depends on the problem data and the initialization of the solid phase, i.e. of $R_{0}$.

Once the positivity of coefficients in 5.52 for $m=s, l$ has been shown under condition (5.57) for all time levels $n_{0} \leq n \leq n_{1}$, where $n_{1}$ is given by (5.60), the stability of the temperatures sign comes from 5.53, obtaining the identity

$$
a_{m, j}^{n}+b_{m, j}^{n}+c_{m, j}^{n}=1, \quad m=s, l .
$$

In fact, from 5.52)-(5.53), 5.61) and the positivity of coefficients $a_{m, j}^{n}, b_{m, j}^{n}, c_{m, j}^{n}$, assuming $-1 \leq u_{s, j}^{n} \leq 0$ and $0 \leq u_{l, j}^{n} \leq 1$, one gets not only the sign preserving but the boundedness property

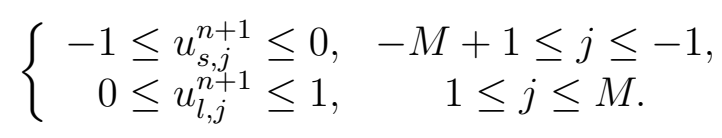

The physics of the problem suggests that the temperatures in both phases increase with the spatial index $j$ for each fixed time level $n, n_{0} \leq n \leq n_{1}$. We show that the numerical solution preserves at this stage the monotonicity property under the stability and positivity conditions (5.57), 5.60) and small enough values of $h$.

From equations (5.52)-(5.53), positivity of coefficients $a_{s, j}^{n}, b_{s, j}^{n}, c_{s, j}^{n}$, and assuming that monotonicity property holds true up to time level $n$, it follows that,

$u_{s, j+1}^{n+1}>\left(\alpha^{n}-\left(1+z_{j+1}\right) \beta^{n}\right) u_{s, j}^{n}+\left(1-\alpha^{n}+\left(1+z_{j+1}\right) \beta^{n}\right) u_{s, j+1}^{n}, \quad-M \leq j \leq-1$, 


\subsection{Front-fixing transformation and numerical modelling}

and

$$
u_{s, j}^{n+1}<\left(1-\alpha^{n}-\left(1+z_{j}\right) \beta^{n}\right) u_{s, j}^{n}+\left(\alpha^{n}+\left(1+z_{j}\right) \beta^{n}\right) u_{s, j+1}^{n}, \quad-M+1 \leq j \leq-1 .
$$

From (5.63) and (5.64) it follows that

$$
u_{s, j+1}^{n+1}-u_{s, j}^{n+1}>\left(u_{s, j+1}^{n}-u_{s, j}^{n}\right)\left(1-2 \alpha^{n}+h \beta^{n}\right)>0, \quad-M+1 \leq j \leq-1 .
$$

In analogous way, under previous conditions and small enough values of $h$, one gets

$$
u_{l, j+1}^{n+1}>u_{l, j}^{n+1}, \quad 0 \leq j \leq M-1 .
$$

Summarizing, the following results have been established in this second stage:

Theorem 10. With previous notation, if the step sizes $h$ and $k$ satisfy the condition (5.57) and for small enough values of $h$, numerical scheme (5.52)-(5.53) and (5.55) preserves the sign, boundedness and the spatial monotonicity of both solid and liquid temperatures in the second stage, starting from the end of the first stage at time level $n_{0}$ and ending at time level $n_{1}$ given by the stability requirement (5.60).

Next example shows temperature profiles in both phases solid and liquid, at the end of the second stage.

Example 2 Figure 5.2 depicts numerical temperature distributions with respect to the variable $z$ at the end of the second stage, i.e. $\tau_{1}=940.481$, both for the solid and the liquid phase. Model parameters take here the values $\kappa=K=\beta=1$, step sizes $h=0.05, k=0.001$ and $R_{0}=0.03$. Note that in this instant, the position of the solidification front is given by the expression $\sigma\left(\tau_{1}\right)=\rho_{0}-\sqrt{\kappa}=32.3333$, i.e. the $97 \%$ of the material is solidified. 


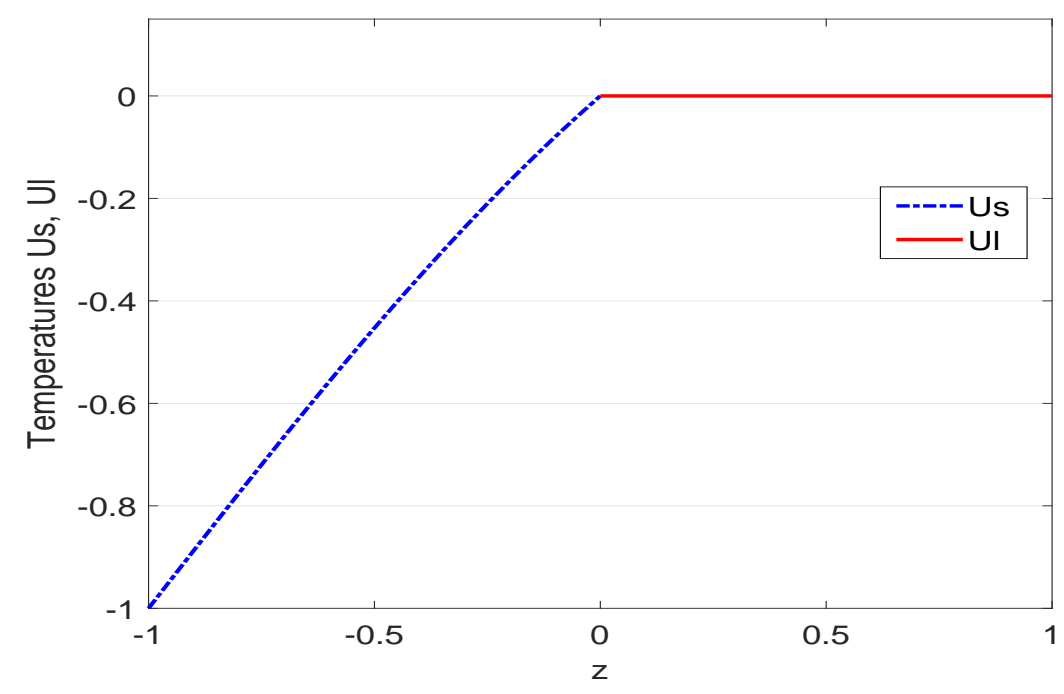

Figure 5.2: Numerical solutions $U_{s}(z, \tau), U_{l}(z, \tau)$ at the end of the second stage.

\subsubsection{Third stage numerical modelling}

As it has been shown at the end of the second stage, the depletion of the liquid phase almost occurs and the temperature remains practically unaltered and close to zero. This fact completes numerically the idea suggested by the authors of [57], p. 270 , about the time $t^{*}$ when the liquid phase can be neglected.

Finally, for the third stage of the problem, within the time range $n_{1} \leq n \leq n_{2}$, where $n_{2}$ corresponds to the time step such that $\sigma^{n_{2}}=\rho_{0}$, i.e. $\tau^{n_{2}}=\tau_{e}$ when the solid phase covers all the domain. As in Section 2.2, the final conditions of the second stage are taken as initial conditions for the third one, while the boundary conditions continue unaltered, (5.37). In this third period, the formulation of the problem is changed by another, characterized only by the equation regarding the solid phase, (5.25), and the Stefan condition, that takes the form

$$
\frac{d \sigma}{d \tau}=\frac{K}{\beta \sigma(\tau)} \frac{\partial U_{s}}{\partial z}, \quad z=0, \quad \tau_{1}<\tau<\tau_{2} .
$$

The solution at the interior points at time level $n+1$, is given by the explicit scheme (5.52) for $m=s$, while the liquid phase is neglected. The properties of the numerical solution established in Theorem 10 remain preserved for the unique solid phase under that unique condition (5.57). 


\subsection{Front-fixing transformation and numerical modelling}

The discretization of the Stefan-like condition (5.67) takes the form

$$
\frac{\sigma^{n+1}-\sigma^{n}}{k}=\frac{K}{\beta \sigma^{n}} \Delta u_{s, 0}^{n}, \quad n_{1} \leq n \leq n_{2}-1 .
$$

In the following example, we illustrate the evolution of temperature profiles during all the stages, showing the properties of spatial monotonicity and stability, as well as the dynamics of the solidification front from the beginning until the extinction time.

Example 3 Figure 5.3 shows the results for the step sizes discretization $h=$ 0.002 and $\Delta \tau=0.01$ in the first stage, verifying conditions (5.43), and $h=0.05$ and $k=0.001$, under the hypothesis of Theorem 10 . The solidified fraction at the end of the first stage is taken $R_{0}=0.03$ and the parameters of the model are $\kappa=K=\beta=1$. Note that each curve corresponds to a different time; the first four upper curves correspond to increasing time values in the second stage, while the last one represents the temperature of the solid phase at the extinction time, i.e. the end of the third stage. The increasing behaviour of the temperatures is captured by the numerical solution and for the liquid phase the temperature is uniformly close to zero in the second stage.

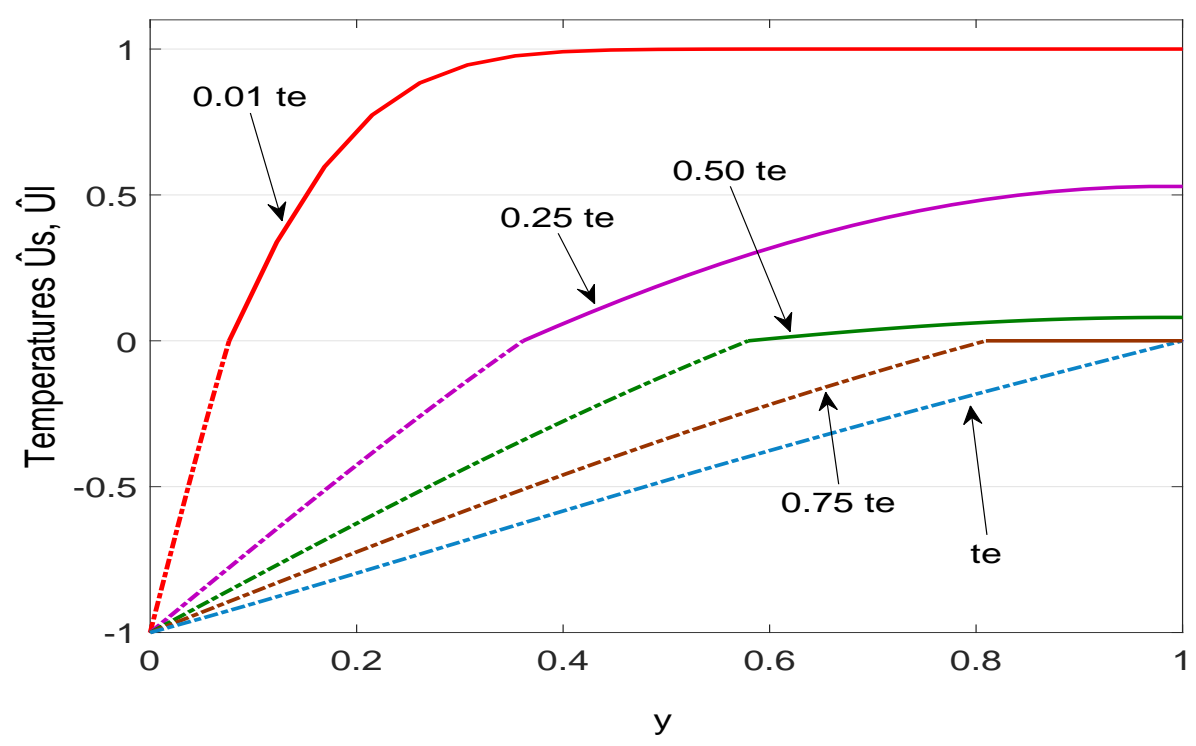

Figure 5.3: Numerical solutions $\hat{U}_{s}(y, \tau), \hat{U}_{l}(y, \tau)$ for different fractions of the extinction time $t_{e}$. Dash-dot lines represent the solid phase and continuous lines the liquid phase. 
Figure 5.4 shows the evolution of the solidification front. It agrees with the results of [57] using the Keller box scheme.

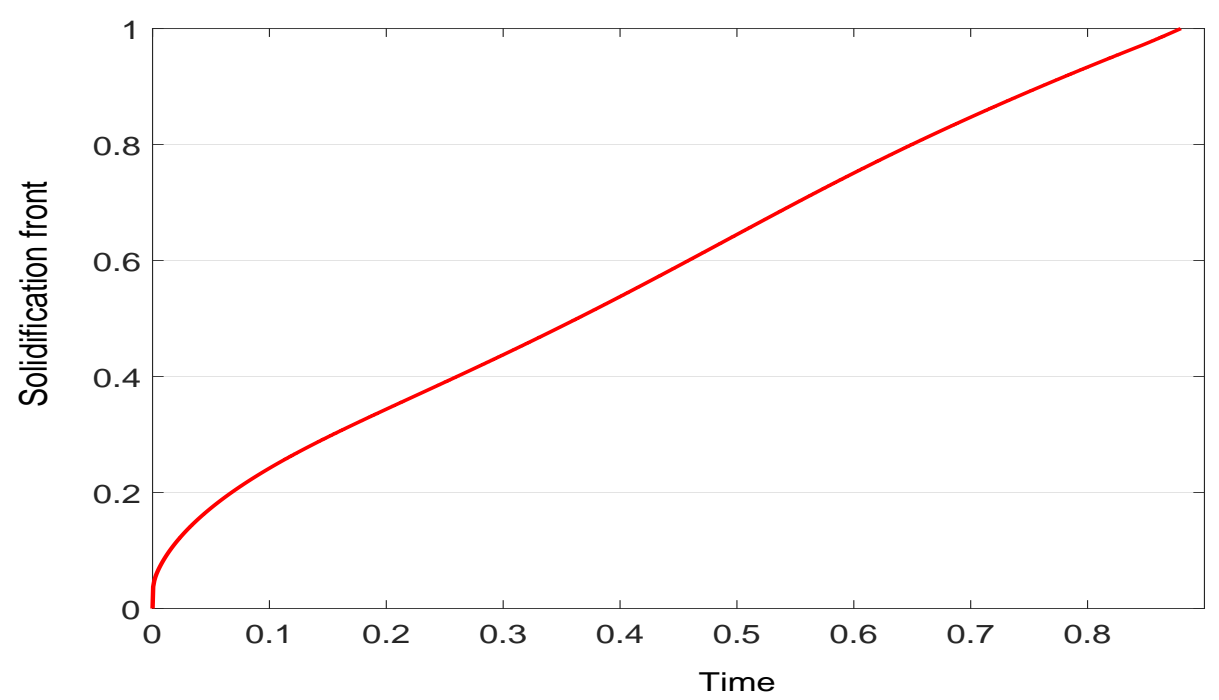

Figure 5.4: Numerical solidification front $\tilde{S}(\tilde{t})$ as a function of time.

Although the first and third stages are qualitative relevant due to the issues of initialization and depletion, it is important to point out that both from the spatial and temporal points of view both stages have a reduced quantitative significance versus the second stage. This facts are illustrated in Figure 5.5, where $R_{0}=0.03$, $t_{e}=0.881195$ and $\tau_{e}=979.106$ for the data in this example. 


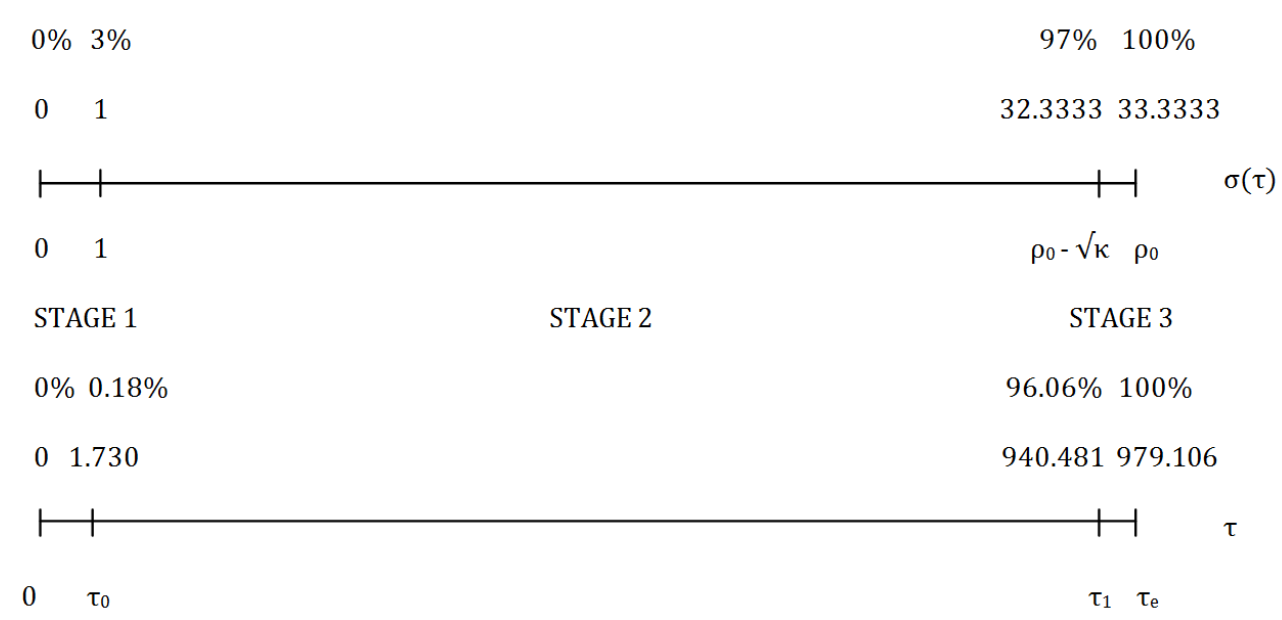

Figure 5.5: Above: Spatial stages distribution. Below: Time stages distribution.

Next example illustrates the fact that both the extinction time and temperatures in the solid phase for complete depletion does not change significantly for changes in a small enough $R_{0}$.

Example 4 Let us take three chosen values of the fraction $R_{0}$. For the model parameters $\kappa=K=\beta=1$ and taking $h=0.002, \Delta \tau=0.01$ in the first stage, $h=0.05, k=0.001$ in the second and third stage, Table 5.2 shows the times $t_{0}$ when finishes the first stage, $t_{1}$ at the end of the second stage and $t_{e}$, as well as the root mean squared error (RMSE) of the distribution of the temperature of the solid at $t_{e}$ with respect to the lower value of $R_{0}=0.01$.

\begin{tabular}{lllllll}
\hline \hline$R_{0}$ & $t_{0}$ & $t_{1}$ & $t_{e}$ & $\%$ 2nd stage & RMSE & $\begin{array}{l}\text { CPU time } \\
\text { (seconds) }\end{array}$ \\
\hline 0.05 & 0.004750 & 0.820648 & 0.876230 & 93.11 & 0.0228 & 79.79 \\
0.03 & 0.001557 & 0.846433 & 0.881195 & 95.88 & 0.0127 & 205.09 \\
0.01 & 0.000103 & 0.873104 & 0.885353 & 98.60 & - & 2067.11 \\
\hline \hline
\end{tabular}

Table 5.2: Dependence of $t_{0}, t_{1}, t_{e}$ and RMSE of temperatures on the fraction $R_{0}$, when the parameters of the model are $\kappa=K=\beta=1$. 



\section{Conclusions}

In this thesis, the numerical solution of parabolic-type moving-boundary problems coming from various fields of science and technology has been obtained. Since most of these problems lack analytical solution, the fact of having approximate solutions is of great interest from a point of view both theoretical and practical. The proposed approach lies in discretizing the continuous problem using finite difference approximations, after immobilizing the boundaries of the continuous problem by applying a suitable transformation. This procedure has been shown to be profitable in dealing with these evolution problems, throughout explicit or implicit schemes that have been implemented in the MATLAB tool, resulting in codes of short computation time.

In all cases, reliable numerical solutions have been obtained, retaining the qualitative properties of the continuous ones. Some essential features as positivity, stability, consistency and monotoncity have been studied by a comprehensive numerical analysis. Furthermore, each numerical scheme have been accompanied by illustrative and enlightening numerical experiments.

In Chapter 2, in which a problem about spreading of invasive biological species has been addressed, the numerical analysis confirms the existence of a spreadingvanishing dichotomy in the long run, and it has been possible to determine, using of numerical experiments, the value of the coefficient in the Stefan condition that 


\section{CONCLUSIONS}

separates the propagation behaviour from the extinction one. Chapters 3 and 4 treat concrete carbonation problems. In Chapter 3 the numerical simulations exhibit the dynamics of the carbonation front and confirm the long-term behaviour as a "square root of time". Chapter 4 comprises the numerical treatment of a more complex and realistic carbonation model, revealing some qualitative characteristics of the solution that are in agreement with the theoretical results. Chapter 5, that focuses on a heat transfer problem arising in industrial solidification processes, presented an additional challenge and the problem was subdivided into three temporal stages to treat the singularities associated with the solidification front position in both the initialisation and depletion stages. Outputs of numerical experiments are consistent with theoretical and numerical results by other authors.

The principal conclusion to be drawn from our approach is that the finite difference approach can be applied successfully to provide numerical solutions of moving-boundary problems. We have been able to obtain valuable insights on the dynamics and solution of each problem. It complements the previous theoretical works, providing numerical approximations for different values of data and model parameters. Against what may be generally accepted about the inadequacy of the explicit method for treating PDE evolution problems which present any type of singularity, it has been extensively used here, revealing that the problem is also tractable by that approach after some appropriate manipulations. Hence, the advantages of the explicit in comparison with implicit methods, i.e. its ease of implementation and less need for computational resources, can be exploited to efficiently treat moving-boundary problems. 


\section{Bibliography}

[1] In Free boundary problems, theory and applications : proceedings of the Zakopane Congress '95, M. Niezgódka and P. Strzelecki, Eds. Addison Wesley Longman, Harlow, Essex, England, United Kingdom, 1996. 91

[2] Aiki, T., And Muntean, A. Large time behavior of solutions to concrete carbonation problem. Comm. Pure Appl. Anal., 9 (2010), 1117-1129. 8, 36, 37

[3] Aiki, T., And Muntean, A. A free-boundary problem for concrete carbonation: rigorous justification of the $\sqrt{t}$-law of propagation. Interfaces and Free Boundaries 15, 2 (2013), 167-180. 8, 36, 37

[4] Aronson, D., And Weinberger, H. Nonlinear diffusion in population genetics, combustion, and nerve pulse propagation. In Partial differential equations and related topics (Program, Tulane Univ., New Orleans, La.), vol. Lecture Notes in Math., Vol. 446. Springer, Berlin, 1975, pp. 5-49. 19

[5] Aronson, D., And Weinberger, H. Multidimensional nonlinear diffusions arising in population genetics. Adv. in Math. 30 (1978), 33-76. 19

[6] Asaithambi, N. A variable time step Galerkin method for a onedimensional Stefan problem. Applied Mathematics and Computation 81, 2 (1997), 189 - 200. 91

[7] Axelsson, O. Iterative Solution Methods. Cambridge University Press, NY, USA, 1994. 99 
[8] Bastidas-Arteaga, E., Schoefs, F., Stewart, M., And Wang, X. Influence of global warming on durability of corroding RC structures: A probabilistic approach. Engineering Structures 51 (2013), 259-266. 55

[9] BeAR, J. Dynamics of Fluids in Porous Media. Dover Publications Inc., New York, 1972. 60

[10] BEAR, J. Biological Invasions: Theory and Practice. Oxford Series in Ecology and Evolution, Oxford University Press, Oxford, 1997. 19

[11] Branco, J., Ferreira, J., AND De Oliveira, P. Numerical methods for the generalized Fisher-Kolmogorov-Petrovskii-Piskunov equation. Applied Numerical Mathematics 57 (2007), 89-102. 20

[12] Briozzo, A., Natale, M., And Tarzia, D. Explicit solutions for a twophase unidimensional Lamé-Clapeyron-Stefan problem with source terms in both phases. Journal of Mathematical Analysis and Applications 329, 1 (2007), 145 - 162. 3, 91

[13] BRown, J.-H. Carbonation. the effect of exposure and concrete quality: field survey results from some 400 structures. In Proc. 5th Int. Conf. Durability of Building Materials and Components. Span Press, London, 1991, pp. 262-271. 36

[14] Bunte, D. Zum Karbonatisierungsbedingten Verlust der Dauerhaftigkeit von Außenbauteilen aus Stahlbeton. $\mathrm{PhD}$ thesis, Technischen Universität Braunschweig, 1994. 36, 56

[15] Bunting, G., Du, Y., And Krakowski, K. Spreading speed revisited: Analysis of a free boundary model. Netw. Heterog. Media 7 (2012), 583603. 21]

[16] Caldwell, J., And Chan, C.-C. Spherical solidification by the enthalpy method and the heat balance integral method. Applied Mathematical Modelling 24, 1 (2000), 45 - 53. 91, 92 
[17] Caldwell, J., AND Kwan, Y. On the perturbation method for the Stefan problem with time-dependent boundary conditions. International Journal of Heat and Mass Transfer 46, 8 (2003), 1497 - 1501. 91

[18] Chantasiriwan, S., Johansson, B., And Lesnic, D. The method of fundamental solutions for free surface Stefan problems. Engineering Analysis with Boundary Elements 33, 4 (2009), 529-538. 91

[19] Chen, X., And Friedman, A. A free boundary problem arising in a model of wound healing. SIAM J. Math. Anal. 32 (2000), 778-800. 19

[20] Churchill, S., AND Gupta, J. Approximations for conduction with freezing or melting. International Journal of Heat and Mass Transfer 20, 11 (1977), 1251 - 1253. 92

[21] Company, R., Egorova, V., And Jódar, L. Solving American option pricing models by the Front fixing method: Numerical analysis and computing. Abstract and Applied Analysis 2014 (2014), 9 pages. 21

[22] Company, R., Egorova, V., And Jódar, L. Constructing positive reliable numerical solution for American call options: A new front-fixing approach. Journal of Computational and Applied Mathematics 291 (2016), 422-431.21

[23] Company, R., Jódar, L., And Pintos, J.-R. A consistent stable numerical scheme for a nonlinear option pricing model in illiquid markets. Mathematics and Computers in Simulation 82, 10 (2012), 1972-1985. 29

[24] CRAnK, J. Two methods for the numerical solution of moving-boundary problems in diffusion and heat flow. Quart. J. Mech. Appl. Math. 10 (1957), 220-231. 91

[25] Crank, J. The Mathematics of Diffusion, 2nd ed. Clarendon Press, Oxford, 1975. 91

[26] Crank, J. Free and Moving Boundary Problems. Oxford Science Publications, 1984. 1, 2, 3, 5, 11, 19, 21, 22, 36, 38, 62, 91, 92, 95, 101 
[27] Danckwerts, P. Gas-liquid Reactions. McGraw-Hill Book Company, New York, 1970. 57

[28] Du, Y., AND GuO, Z. Spreading-vanishing dichotomy in the diffusive logistic model with a free boundary, II. J. Diff. Eqns. 250 (2011), 4336-4366. 20, 34

[29] Du, Y., AND Guo, Z. The Stefan problem for the Fisher-KPP equation. $J$. Diff. Eqns. 253 (2012), 996-1035. 20

[30] DU, Y., AND LIN, Z. Spreading-vanishing dichotomy in the diffusive logistic model with a free boundary. SIAM J. Math. Anal. 42, 1 (2010), 377-405. 6 , 19, 20, 21, 26, 27, 31, 33, 34

[31] Duffy, D. Finite Difference Methods in Financial Engineering: A Partial Differential Equation Approach. John Wiley \& Sons, 2006. 19

[32] Esen, A., And Kutluay, S. A numerical solution of the Stefan problem with a Neumann-type boundary condition by enthalpy method. Applied Mathematics and Computation 148, 2 (2004), 321 - 329. 92

[33] Fatima, T., And Muntean, A. Sulfate attack in sewer pipes: Derivation of a concrete corrosion model via two-scale convergence. Nonlinear Analysis: Real World Applications 15, 1 (2014), 326-344. 55

[34] Fisher, R. The wave of advance of advantageous genes. Ann. Eugenics 7 (1937), 335-369. 6, 19

[35] Godunov, S. A difference scheme for numerical solution of discontinuous solution of hydrodynamic equations. Math. Sbornik 47 (1959), 271-306. 48 , 82

[36] Goodman, T. The heat-balance integral and its application to problems involving a change of phase. Trans. ASME 80, 2 (1958), 335-342. 91

[37] Groves, G.-W., Brough, A., Richardson, I.-G., And Dobson, C.M. Progressive changes in the structure of hardened $\mathrm{C}_{3} \mathrm{~S}$ cement pastes due to carbonation. J. American Ceramic Society 11, 74 (1991), 2891-2896. 36 
[38] Gupta, S. The Classical Stefan Problem, Volume 45, 1st Edition. Basic concepts, modelling and analysis. JAI Press, 2003. 91

[39] Haferkamp, H., Mai, T., And Marquering, M. Hybrid method for numerical solution of moving boundary problems in laser surface remelting. Computational Mechanics Publications 3rd Int. Conf. Computational Modelling of Free and Moving Boundary Problems III (1995), 371-378. 3

[40] Hon, Y. C., AND LI, M. A computational method for inverse free boundary determination problem. International Journal for Numerical Methods in Engineering 73, 9 (2008), 1291-1309. 91

[41] Ishida, T., Maekawa, K., And Soltani, M. Theoretically identified strong coupling of carbonation rate and thermodynamic moisture states in micropores of concrete. Journal of Advanced Concrete Technology 2, 2 (2004), 213-222. 36, 56

[42] Kishitani, Y., Hokoi, S., Harada, K., and Takada, S. Prediction model for carbonation of concrete structures considering heat and moisture transfer. J. Structural and Construction Engineering Trans. AIJ 595 (2005), 17-23. 36, 56

[43] Kolmogorov, A., Petrovsky, N., And Piskunov, S. étude de l'équations de la diffusion avec croissance de la quantité de matière et son application a un problème biologique. Bull. Univ. Moskou 1 (1937), 1-25. 6 . 19

[44] Kröner, D. Numerical Schemes for Conservation Laws. Wiley-Teubner Series, 1997. 29

[45] Kumazaki, K. A mathematical model of carbon dioxide transport in concrete carbonation process. Discrete and Continuous Dynamical Systems Series $S$ 7, 1 (2014), 113-125. 56

[46] Kutluay, S., BAhadir, A., And Özdeş, A. The numerical solution of one-phase classical Stefan problem. Journal of Computational and Applied Mathematics 81, 1 (1997), 135-144. 91 
[47] Kutluay, S., And Esen, A. An isotherm migration formulation for onephase Stefan problem with a time dependent Neumann condition. Applied Mathematics and Computation 150, 1 (2004), 59 - 67. 92

[48] Lamé, G., And Clapeyron, B. Memoire surla solidification par refroidissement d'un globe liquid. Annales Chimie Physique 47 (1831), 250-256. 2

[49] Landau, H.-G. Heat condution in a melting solid. Quarterly of Applied Mathematics 8 (1950), 81-95. 3, 5, 21, 22, 38, 62, 92, 95

[50] Liang, X., AND ZhaO, X.-Q. Asymptotic speeds of spread and traveling waves for monotone semiflows with applications. Comm. Pure Appl. Math. 60 (2007), 1-40. 19

[51] LogAn, J. Transport Modeling in Hydrogeochemical Systems. Interdisciplinary Applied Mathematics. Springer New York, 2001. 60

[52] MacíAs-DíAz, J., AND PURI, A. An explicit positivity-preserving finitedifference scheme for the classical Fisher-Kolmogorov-Petrovsky-Piskunov equation. Applied Mathematics and Computation 218 (2012), 5829-5837. 20

[53] Mainguy, M., And Coussy, O. Propagation fronts during calcium leaching and chloride penetration. Journal of Engineering Mechanics, ASCE 126, 3 (2000), 250-257. 57

[54] Meek, P., ANd Norbury, J. Nonlinear moving boundary problems and a Keller box scheme. SIAM Journal on Numerical Analysis 21, 5 (1984), 883-893. 92

[55] Mitchell, S., And Vynnycky, M. Finite-difference methods with increased accuracy and correct initialization for one-dimensional Stefan problems. Appl. Math. Comput. 215, 4 (2009), 1609-1621. 95

[56] Mitchell, S., And Vynnycky, M. The oxygen diffusion problem: Analysis and numerical solution. Applied Mathematical Modelling 39, 9 (2015), 2763-2776. 91 
[57] Mitchell, S., AND VynnyCKY, M. On the accurate numerical solution of a two-phase Stefan problem with phase formation and depletion. J. Comput. Appl. Math. 300, C (2016), 259-274. 10, 92, 96, 106, 108

[58] Muntean, A. A Moving-Boundary Problem: Modeling, Analysis and Simulation of Concrete Carbonation. PhD thesis, Faculty of Mathematics, University of Bremen, 2006. 37, 56

[59] Muntean, A., And BöHm, M. A moving-boundary problem for concrete carbonation: Global existence and uniqueness of weak solutions. Journal of Mathematical Analysis and Applications 350 (2009), 234-251. 9, 39, 56, 57. 58, 70, 72, 79, 87

[60] Muntean, A., BÖHm, M., And Kropp, J. Moving carbonation fronts in concrete: A moving-sharp-interface approach. Chemical Engineering Science 66, 3 (2011), 538-547. 9, 37, 45, 51, 56, 57, 58, 60, 80, 87

[61] Papadakis, V., Vayenas, C., And FARdis, M. A reaction engineering approach to the problem of concrete carbonation. AIChE Journal 35, 10 (1989), 1639-1650. 36, 56

[62] Plemmons, R. M-matrix characterizations. I-Nonsingular M-matrices. Linear Algebra and its Applications 18, 2 (1977), 175 - 188. 99

[63] QIn, W., Ding, D., AND Ding, X. Two boundedness and monotonicity preserving methods for a generalized fisher-KPP equation. Applied Mathematics and Computation 252 (2015), 552-567. 20

[64] Ramezanianpour, A., Ghahari, S., And Esmaeili, M. Effect of combined carbonation and chloride ion ingress by an accelerated test method on microscopic and mechanical properties of concrete. Construction and Building Materials 58 (2014), 138-146. 55

[65] RizWAN-UDDin. A nodal method for phase change moving boundary problems. International Journal of Computational Fluid Dynamics 11, 3-4 (1999), 211-221. 91 
[66] Rubinstein, L. The Stefan problem, Translations of mathematical monographs. American Mathematical Society, Providence, RI 27 (1971), 52-55. 2. 19

[67] Russell, D., Basheer, P., Rankin, G., And Long, A. Effect of relative humidity and air permeability on prediction of the rate of carbonation. Structures \& Buildings 3, 146 (2001), 319-326. 36

[68] Savović, S., AND CAldwell, J. Finite difference solution of onedimensional Stefan problem with periodic boundary conditions. International Journal of Heat and Mass Transfer 46, 15 (2003), 2911-2916. 91, 92

[69] Schmidt, A., Muntean, A., And BÖHm, M. Numerical experiments with self-adaptive finite element simulations in $2 \mathrm{~d}$ for the carbonation of concrete. 88

[70] Sisomphon, K. Influence of pozzolanic material additions on the development of the alkalinity and the carbonation behaviour of composite cement pastes and concretes. $\mathrm{PhD}$ thesis, TU Hamburg, 2004. 36

[71] Skellam, J. Random dispersal in theoretical populations. Biometrika 38 (1951), 196-218. 19.

[72] Smith, G. Numerical Solution of Partial Differential Equations: Finite Difference Methods, 3rd ed. Clarendon Press, Oxford, 1985. 13, 14, 24, 25, 51, 52, 98

[73] Solomon, S. Climate change 2007-the physical science basis: Working group I contribution to the fourth assessment report of the IPCC, vol. 4. Cambridge University Press, 2007. 55

[74] Stefan, J. S-B Wien Akad. Mat. Natur. 98 (1889), 473-484, 965-983. 2

[75] Stephan, K., And Holzknecht, B. Perturbation solutions for solidification problems. International Journal of Heat and Mass Transfer, 19 (2017), 597-602. 91 
[76] STRIKWERDA, J.-C. Finite difference schemes and partial differential equations, 2nd ed. SIAM: Society for Industrial and Applied Mathematics, 2004. 51

[77] TARZIA, D. Relationship between Neumann solutions for two-phase LaméClapeyron-Stefan problems with convective and temperature boundary conditions. Thermal Science 21, 1A (2017), 187 - 197. 95,96

[78] Taylor, H. Cement Chemistry. Thomas Telford Publishing, London, 1997. 60

[79] TuUtTi, K. Corrosion of steel in concrete. Swedish Cement and Concrete Research Institute (CBI), Stockholm, 1982. 36, 56

[80] Uоmoto, T., AND TAKAdA, Y. Factors affecting concrete carbonation rate. Durability of Building Materials Components 6 (1993), 1133-1141. 36, 56

[81] Voller, V., ANd Cross, M. Applications of control volume enthalpy methods in the solution of Stefan problems. In Computational Techniques in Heat Transfer, R. Lewis, K. Morgan, J. Johnson, and W. Smith, Eds. Pineridge Press Ltd., Mambles, Swansea, UK, 1985. 92

[82] WANG, M. The diffusive logistic equation with a free boundary and signchanging coefficient. J. Diff. Eqns. 258, 4 (2015), 1252-1266. 20

[83] Weinberger, H. F. On spreading speeds and traveling waves for growth and migration models in a periodic habitat. J. Math. Biol. 45 (2002), 511-548. 19

[84] Weinberger, H. F., Lewis, M., And Li, B. Anomalous spreading speeds of cooperative recursion systems. J. Math. Biol. 55 (2007), 207-222. 19

[85] Yoon, I. Deterioration of concrete due to combined reaction of carbonation and chloride penetration: Experimental study. Key Engineering Materials 348-349 (2007), 729-732. 55 


\section{Declaration}

I herewith declare that I have produced this work without the prohibited assistance of third parties and without making use of aids other than those specified; notions taken over directly or indirectly from other sources have been identified as such. This work has not previously been presented in identical or similar form to any examination board.

The dissertation work was conducted from 2015 to 2018 under the supervision of Dr. Lucas Jódar Sánchez and Dr. Rafael Company Rossi at the Polytechnic University of Valencia.

Valencia, 
This dissertation was finished writing in Valencia on Thursday $15^{\text {th }}$ February, 2018 
This page is intentionally left blank 